LBL-37334

UC-401

\title{
Synthesis, Structure, and Reactivity of High Oxidation State Silver Fluorides and Related Compounds
}

\author{
George Michael Lucier \\ Ph.D. Thesis \\ Department of Chemistry \\ University of California, Berkeley \\ and \\ Chemical Sciences Division \\ Lawrence Berkeley Laboratory \\ University of California \\ Berkeley, CA 94720
}

May 1995

This work was supported by the Director, Office of Energy Research, Office of Basic Energy Sciences, Materials Sciences Division, of the U.S. Department of Energy under Contract No. DE-AC03-76SF00098. 



\section{DISCLAIMER}

This report was prepared as an account of work sponsored by an agency of the United States Government. Neither the United States Government nor any agency thereof, nor any of their employees, make any warranty, express or implied, or assumes any legal liability or responsibility for the accuracy, completeness, or usefulness of any information, apparatus, product, or process disclosed, or represents that its use would not infringe privately owned rights. Reference herein to any specific commercial product, process, or service by trade name, trademark, manufacturer, or otherwise does not necessarily constitute or imply its endorsement, recommendation, or favoring by the United States Government or any agency thereof. The views and opinions of authors expressed herein do not necessarily state or reflect those of the United States Government or any agency thereof. 


\section{DISCLAIMER}

Portions of this document may be illegible in electronic image products. Images are produced from the best available original document. 
Synthesis, Structure, and Reactivity of High Oxidation State Silver Fluorides and Related Compounds

by

George Michael Lucier

B.S. (Stanford University) 1990

A dissertation submitted in partial satisfaction of the requirements for the degree of

Doctor of Philosophy

in

Chemistry

in the

GRADUATE DIVISION

of the

UNIVERSITY of CALIFORNIA at BERKELEY

Committee in charge:

Professor Neil Bartlett, Chair

Professor Angelica Stacy

Professor Alex Zettl 


\begin{abstract}
Synthesis, Structure, and Reactivity of

by

George Michael Lucier

Doctor of Philosophy in Chemistry

University of California at Berkeley

Professor Neil Bartlett, Chair
\end{abstract}

High Oxidation State Silver Fluorides and Related Compounds

The research described in this thesis has been largely concerned with defining the oxidizing power of $\mathrm{Ag}(\mathrm{III})$ and $\mathrm{Ag}(\mathrm{II})$ species in anhydrous hydrogen fluoride (aHF) solution. Emphasis has been placed on the study of cationic species, since in a cation the electronegativity of a given oxidation state is greater than in the neutral or anionic relatives. Cationic $\mathrm{Ag}_{\text {solv }}^{\mathrm{III}}$ has a short half life at ordinary temperatures, oxidizing the solvent to elemental fluorine with formation of $\mathrm{Ag}(\mathrm{II})$. Salts of such a cation have not yet been preparable, but solutions which must contain such a species have proved to be effective and powerful oxidizers. In the presence of $\mathrm{PtF}_{6}^{-}, \mathrm{RuF}_{6}^{-}$, or $\mathrm{RhF}_{6}^{-}, \mathrm{Ag}_{\text {solv }}^{\mathrm{II}}$ effectively oxidizes the anions to release the neutral hexafluorides. Such reactivity ranks cationic $\mathrm{Ag}(\mathrm{III})$ as the most powerfully oxidizing chemical agent known so far. Unlike its trivalent relative, $\mathrm{Ag}_{\text {solv }}^{\mathrm{II}}$ is thermodynamically stable in acid aHF. Nevertheless, it has been observed to oxidize $\operatorname{IrF}_{6}^{-}$to $\operatorname{IrF}_{6}$ at room temperature, placing its 
oxidizing potential not more than $2 \mathrm{eV}$ below that of cationic $\mathrm{Ag}(\mathrm{III})$. The range of $\mathrm{Ag}^{2+}\left(\mathrm{MF}_{6}\right)_{2}$ salts attainable in aHF has been explored. An anion must be stable with respect to loss of its electron to $\mathrm{Ag}^{2+}$. The anion must also be a poor $\mathrm{F}^{-}$donor; otherwise, either $\mathrm{AgF}^{+}$salts or $\mathrm{AgF}_{2}$ are generated.

A general method for the synthesis of $\mathrm{AgF}^{+} \mathrm{MF}_{6}^{-}$salts has been found in which $\mathrm{Ag}^{+} \mathrm{MF}_{6}^{-}$salts are fluorinated in aHF. $\mathrm{AgFIrF} \mathrm{F}_{6}$ is preparable, but $\mathrm{AgFOsF}_{6}$ is not, placing the oxidizing potential of the polymeric fluorine-bridged $(\mathrm{AgF})_{n}^{\mathrm{n}+}$ cation $\sim 1 \mathrm{eV}$ lower than that of $\mathrm{Ag}_{\text {solv. }}^{\mathrm{II}}$. The $\mathrm{AgF}^{+} \mathrm{MF}_{6}^{-}$salts have been found to adopt at least three different structure types, ranging from monoclinic to tetragonal symmetry. The structures of $\mathrm{AgF}^{+} \mathrm{BF}_{4}^{-}$and $\mathrm{AgF}^{+} \mathrm{IrF}_{6}^{-}$reveal a polymeric chain cation of formula $(\mathrm{AgF})_{\mathrm{n}}^{\mathrm{n}+}$ in which the $\mathrm{Ag}(\mathrm{II})$ is linearly coordinated. $\mathrm{A}$ second $\mathrm{AgF}^{+} \mathrm{MF}_{6}^{-}$structure type occurs in $\mathrm{AgF}^{+} \mathrm{RuF}$, which exhibits square coordination of the cation, generating a one-dimensional ribbon. The $\mathrm{AgF}^{+} \mathrm{MF}_{6}^{-}(\mathrm{M}=\mathrm{Sb}, \mathrm{Bi})$ represent yet another structure type (based on X-ray powder data), but the atomic arrangement is not yet known. All $\mathrm{AgF}^{+}$salts examined exhibit a small temperature-independent paramagnetism indicative of a metallic band structure (i.e. Pauli paramagnetism). Polarized light transmission experiments on single crystals of $\mathrm{AgFBF}_{4}$ support the hypothesis that these salts are one-dimensional metals, but no direct evidence for electronic conductivity has been found. 


\begin{abstract}
Synthesis, Structure, and Reactivity of
High Oxidation State Silver Fluorides and Related Compounds
\end{abstract}

\title{
Table of Contents
}

\section{Chapter 1 Introduction and Experimental Considerations .........................................1}

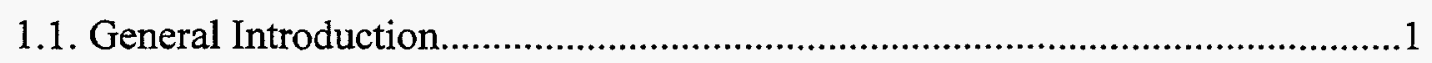

1.2. Electron Affinities of Third Row Transition Metal Hexafluorides .......................

1.3. Relative Fluoride Ion Affinities of Some Fluoro-acids ........................................

1.3.1. Fluoro-acidity Ranking of Main Group Fluorides...................................6

1.4. Stabilization of High Oxidation State Cations Such as $\mathrm{AgF}_{2}^{+} \ldots \ldots \ldots \ldots \ldots \ldots \ldots \ldots \ldots . . .9$

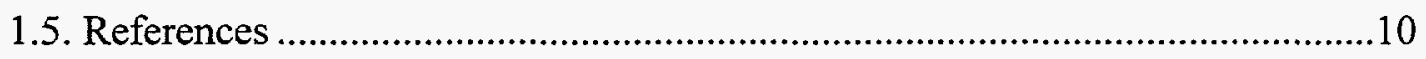

2. Chapter 2 Experimental Methods ..................................................................................12

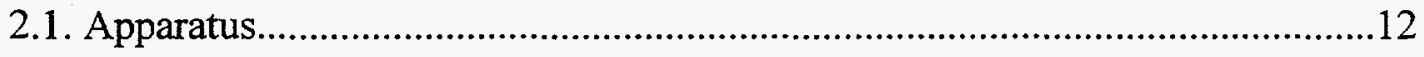

2.2. Characterization Techniques ........................................................................13

2.2.1. X-ray Powder Diffraction .................................................................13

2.2.2. Single Crystal X-ray Diffraction.............................................................14

2.2.3. SQUID Magnetometry ....................................................................... 14

2.3. Anhydrous $\mathrm{HF}$ as an Oxidatively Inert Solvent ...............................................14

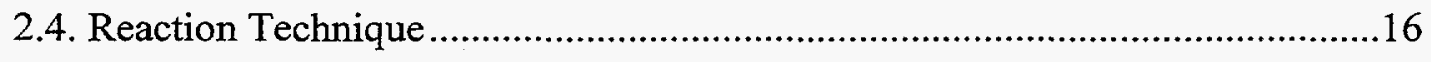

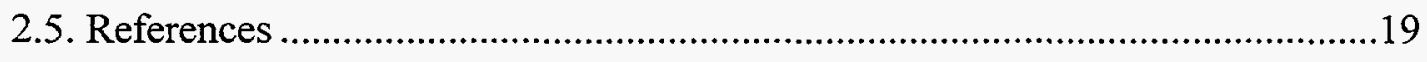

3. Chapter 3 Preparation of Reagent Materials .........................................................20 
3.1. Introduction

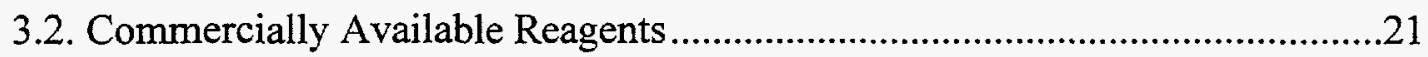

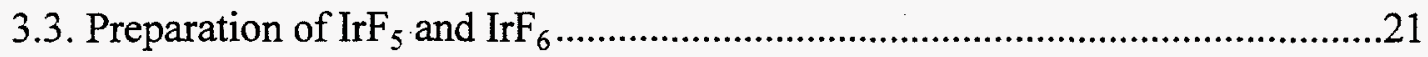

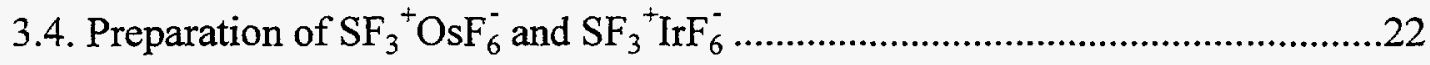

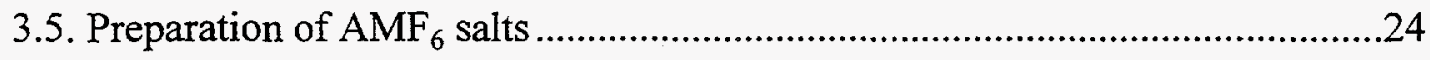

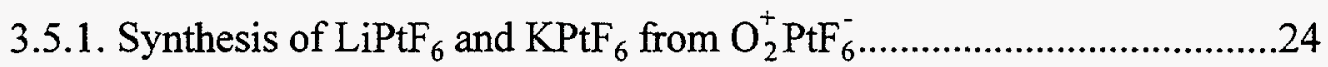

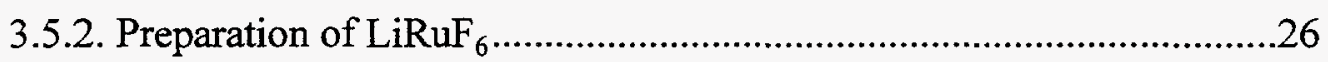

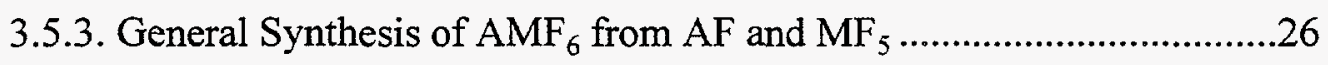

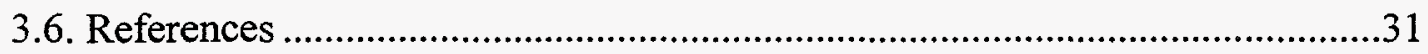

4. Chapter 4 The Oxidizing Chemistry of Solvated $\mathrm{Ag}^{\mathrm{III}}$...........................................32

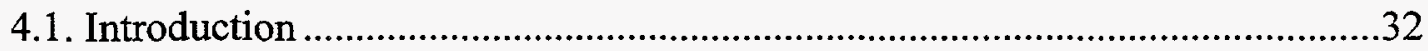

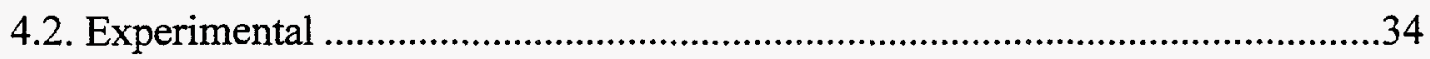

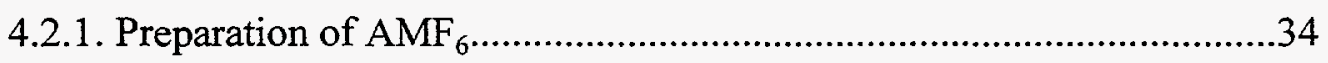

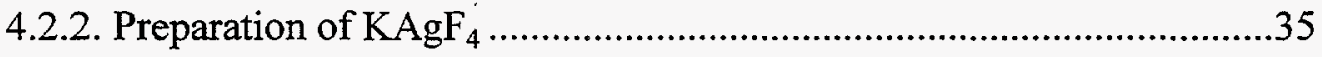

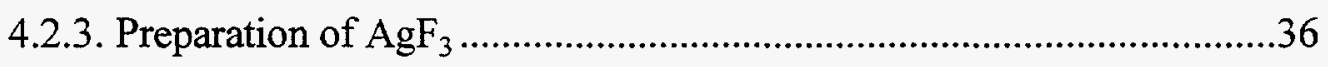

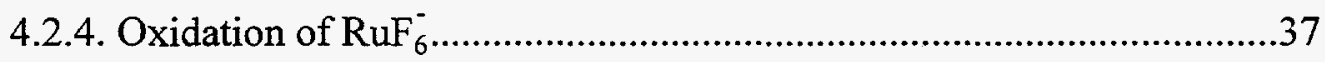

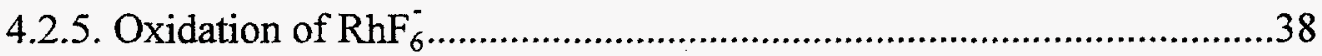

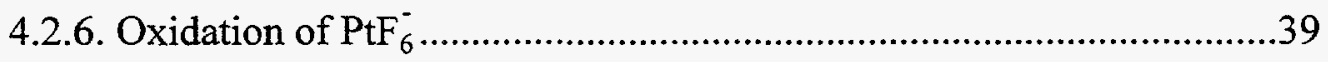

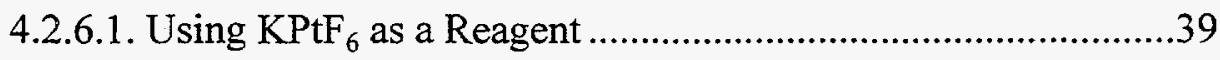

4.2.6.2. Using $\mathrm{LiPtF}_{6}$ as a Reagent ...................................................... 
4.2.7. Attempted Oxidation of $\mathrm{AuF}_{6}^{-}$

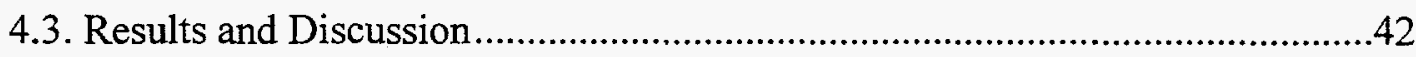

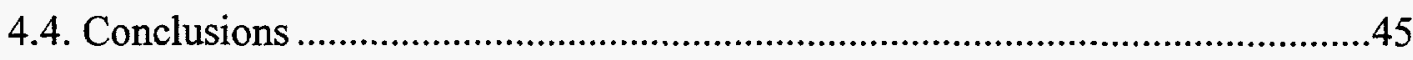

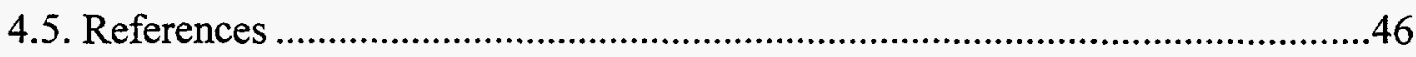

5. Chapter 5 Synthesis and Oxidizing Properties of $\mathrm{AgF}^{+}$Salts ................................47

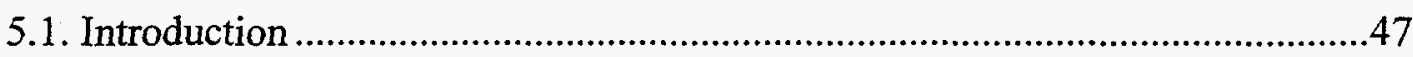

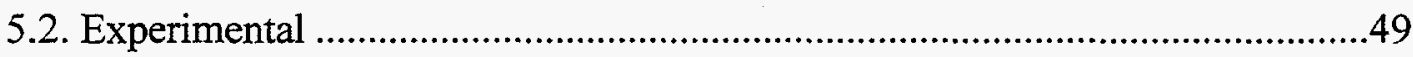

5.2.1. Interaction of $\mathrm{LiOsF}_{6}$ with $\mathrm{F}_{2}$ in aHF ...........................................49

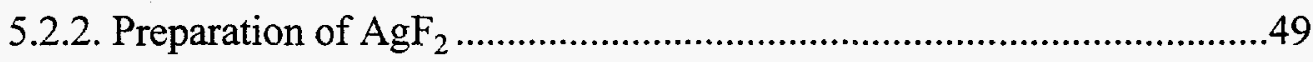

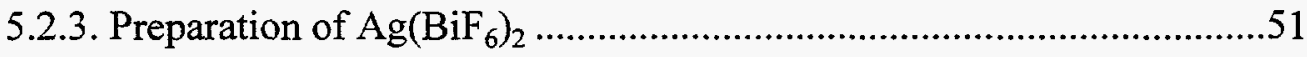

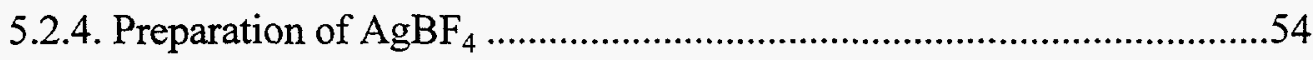

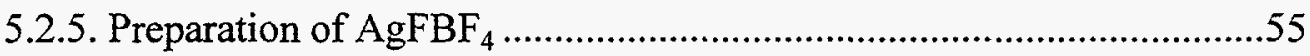

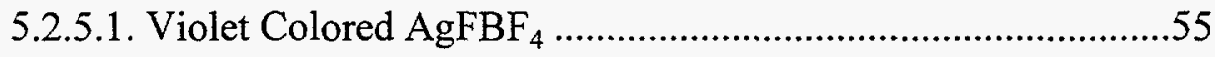

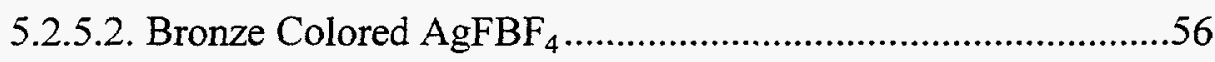

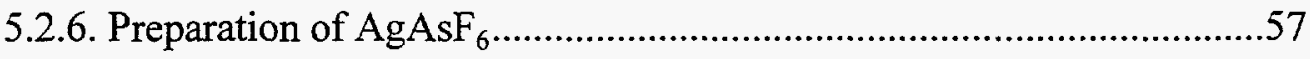

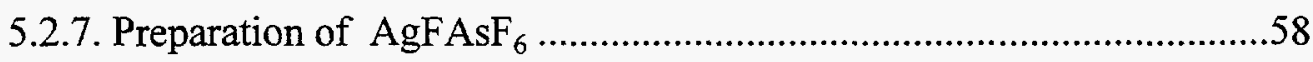

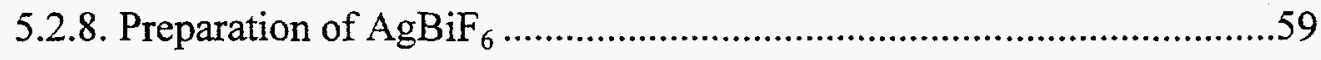

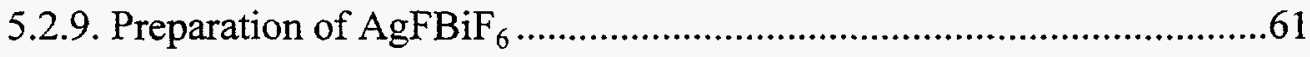

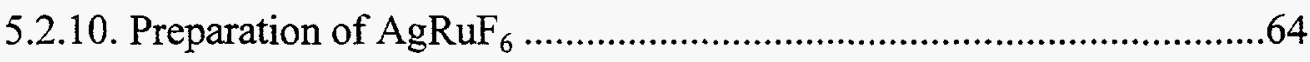

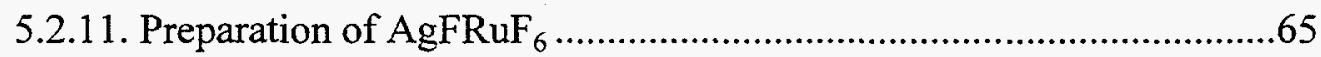




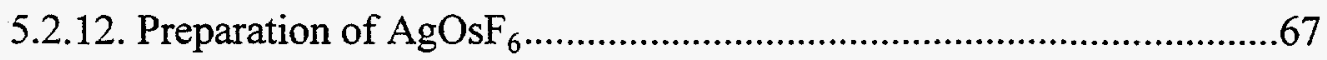

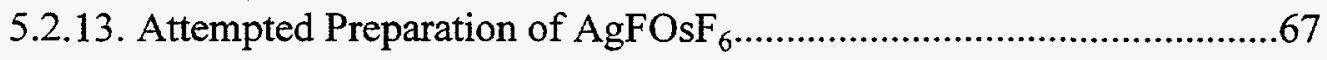

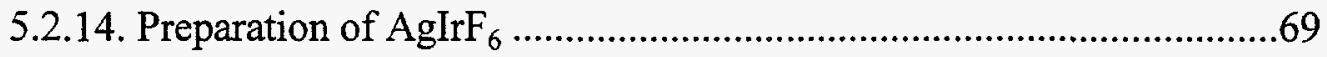

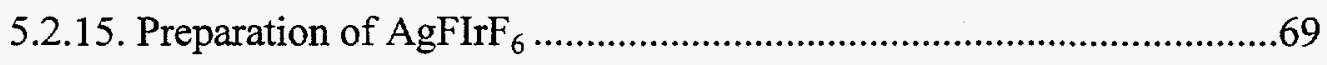

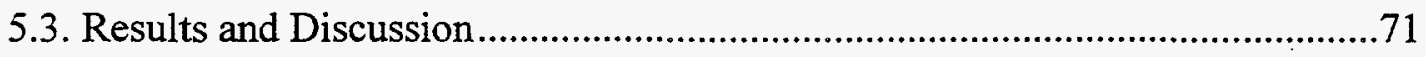

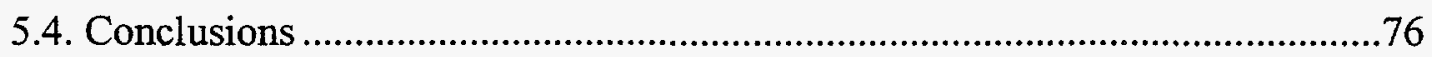

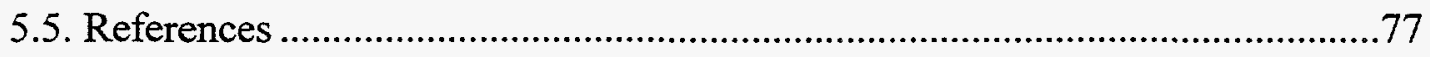

6. Chapter 6 Structure and Physical Properties of $\mathrm{AgF}^{+}$Salts ....................................79

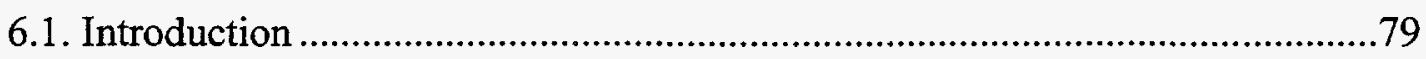

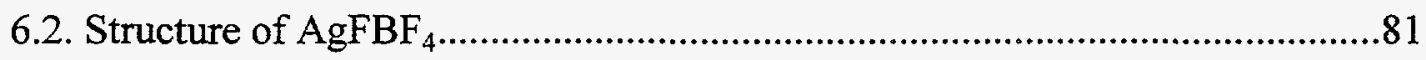

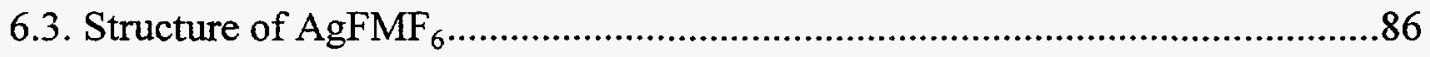

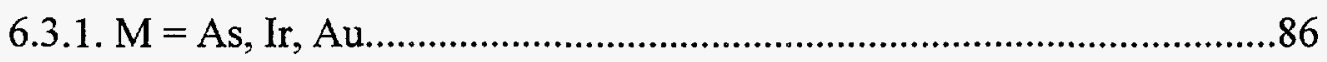

6.3.2. $\mathrm{M}=\mathrm{Sb}, \mathrm{Bi}$

6.3.3. $\mathrm{M}=\mathrm{Ru}$

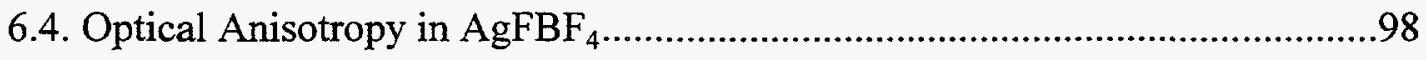

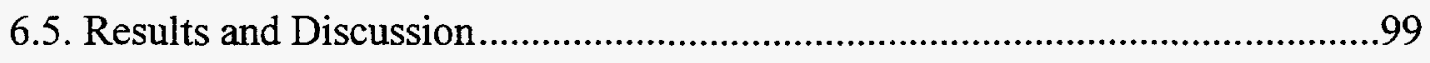

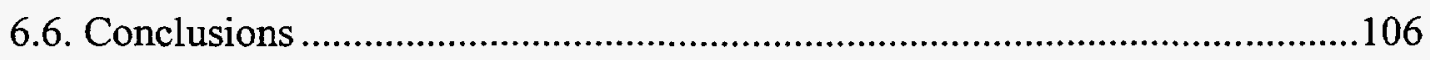

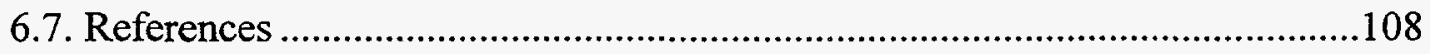

7. Chapter 7 The Oxidizing Chemistry of Solvated $\mathrm{Ag}^{\mathrm{II}}$.............................................110

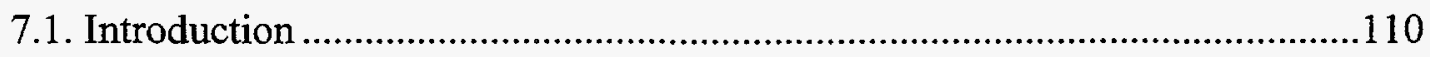

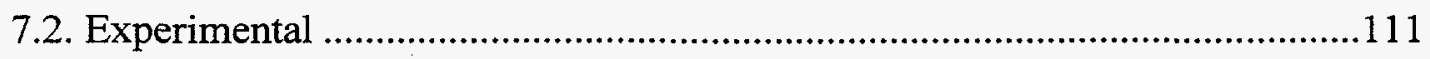


7.2.1. Preparation of $\mathrm{AMF}_{6}$

7.2.2. Interaction of $\mathrm{KIrF}_{6}$ with $\mathrm{F}_{2}$ in Acidic aHF

7.2.3. Oxidation of $\operatorname{IrF}_{6}^{-}$with $\mathrm{AgFAsF}_{6}$ in acidic aHF

7.2.4. Oxidation of $\operatorname{IrF}_{6}^{-}$with $\mathrm{Ag}\left(\mathrm{SbF}_{6}\right)_{2}$

7.2.5. Interaction of $\mathrm{AgFIrF}_{6}$ with $\mathrm{BiF}_{5}$ in the Presence of $\mathrm{F}_{2}$

7.2.6. Attempted Oxidation of $\mathrm{PtF}_{6}^{-}$

7.3. Results and Discussion

7.4. Conclusions

7.5. References

8. Chapter $8 \quad \operatorname{Ag}^{\mathrm{I}} \mathbf{M}^{\mathrm{V}} \mathrm{F}_{6}$ vs. $\operatorname{Ag}^{\mathrm{II}} \mathbf{M}^{\mathrm{IV}} \mathrm{F}_{6}$

8.1. Introduction

8.2. Experimental

8.2.1. Preparation of some $A_{2} M_{6}$ salts from $A F$, metallic $M$, and $F_{2} \ldots \ldots \ldots .120$

8.2.2. Preparation of $\mathrm{AgPtF}_{6}$

8.2.3. Preparation of $\mathrm{AgPdF}_{6}$

8.2.4. Interaction of $\mathrm{AgF}$ with $\mathrm{KPtF}_{6}$

8.2.5. Interaction of $F_{2}$ with $\mathrm{AgPt}_{6}$ and $\mathrm{AgPdF}_{6}$ 125

8.2.6. Interaction of $\mathrm{O}_{2} \mathrm{AsF}_{6}$ with $\mathrm{PtF}_{6}^{2-}$ 125

8.2.7. Interaction of $\mathrm{O}_{2} \mathrm{AsF}_{6}$ with $\mathrm{PdF}_{6}^{2-}$ 126

8.2.8. Interaction of $\mathrm{K}_{2} \mathrm{PdF}_{6}$ with $\mathrm{PF}_{5}$ 
viii

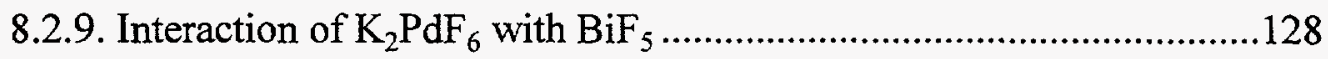

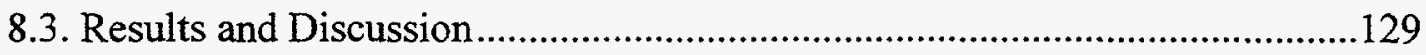

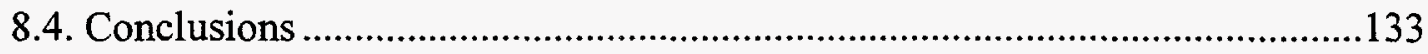

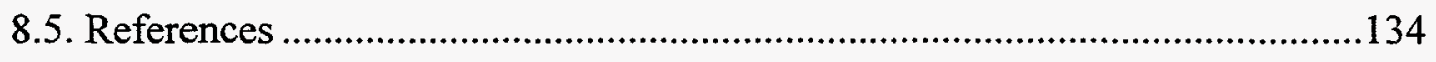

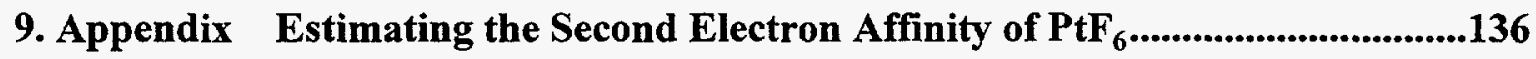

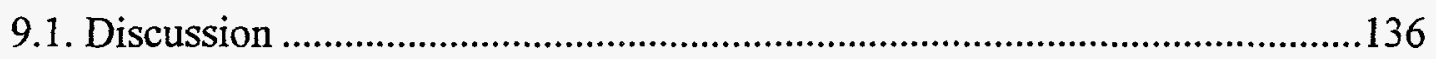

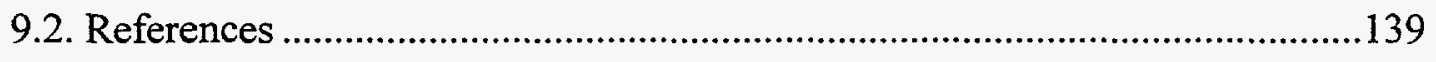




\section{List of Figures}

Figure 1.1. Electron Affinities of Third Row Transition Metal Hexafluorides. ...............4

Figure 2.1. A typical reactor tube for use with anhydrous HF solution chemistry..........17

Figure 5.1. Magnetic data for mixture of $15 \% \mathrm{AgF}_{2}, 85 \% \mathrm{CaF}_{2} \ldots \ldots \ldots \ldots \ldots \ldots \ldots \ldots \ldots \ldots . . . . . \ldots 1$

Figure 5.2. Magnetic data for $\mathrm{AgFBF}_{4}$. ..............................................................56

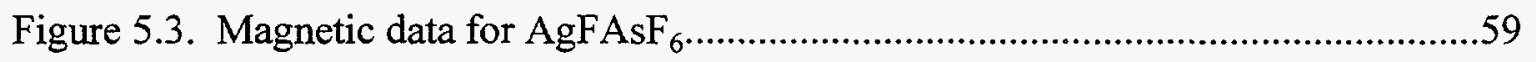

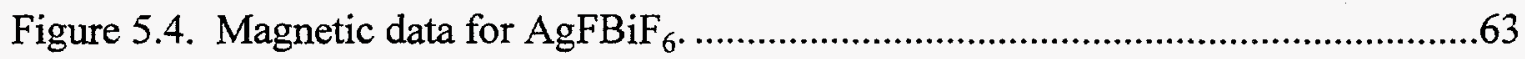

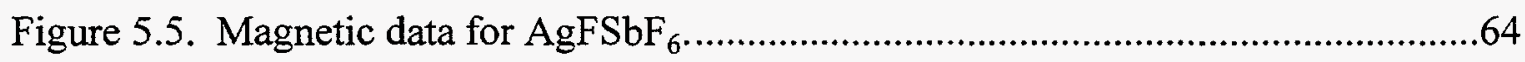

Figure 5.6. Magnetic data for $\mathrm{AgRuF}_{6}$, indicating a simple three-electron paramagnet. .65

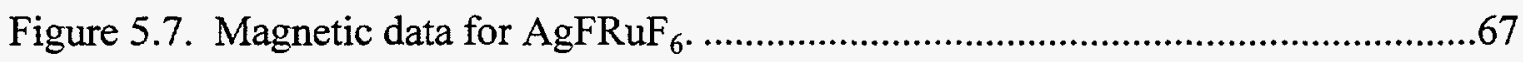

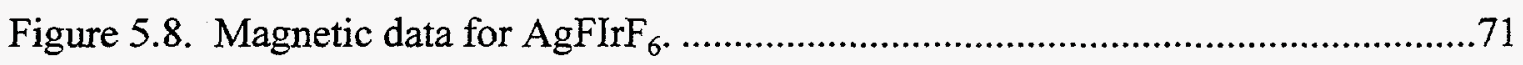

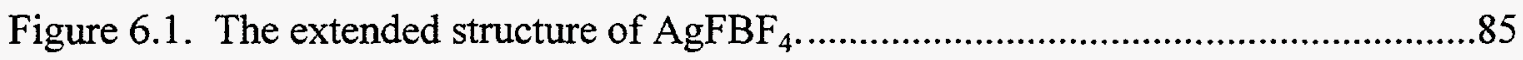

Figure 6.2. The tetragonally-distorted octahedral environment about the $\mathrm{Ag}(\mathrm{II})$ in

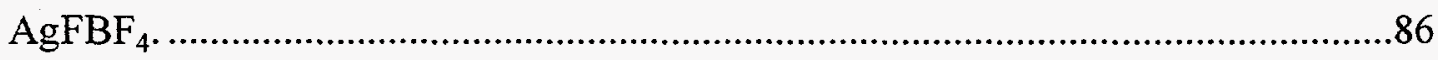

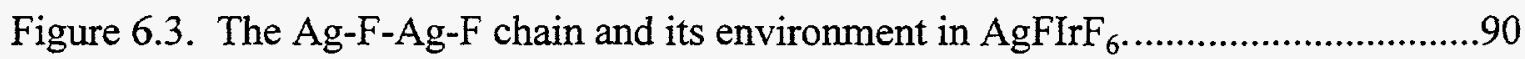

Figure 6.4. The Ag(II) and $\operatorname{Ir}(\mathrm{V})$ structural environments in $\mathrm{AgFIrF}_{6} \ldots \ldots \ldots \ldots \ldots \ldots \ldots \ldots . . . . . . . . . . .1$

Figure 6.5. The structure of $\mathrm{AgFRuF}_{6}$, showing the one-dimensional ribbon cationic

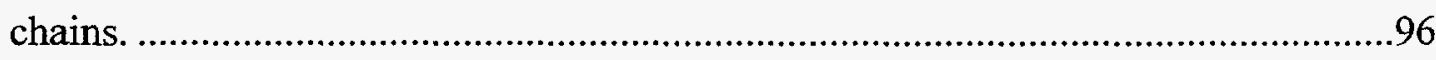

Figure 6.6. An ORTEP displaying the square-planar bonding to silver in $\mathrm{AgFRuF}_{6} \ldots . . .97$

Figure 6.7. An ORTEP depicting the puckered planes of $\mathrm{AgF}_{2}, \ldots \ldots \ldots \ldots \ldots \ldots \ldots \ldots \ldots \ldots . . . . . . \ldots 8$

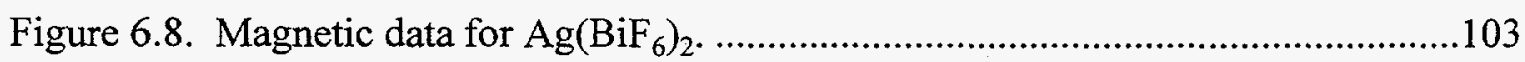




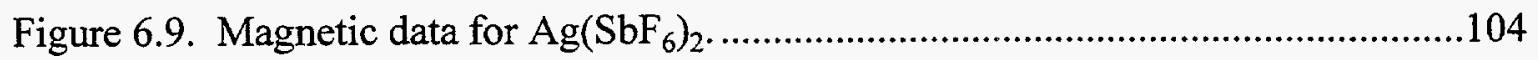

Figure 8.1. Magnetic data for $\mathrm{AgPdF}_{6}$ indicating simple paramagnetic behavior..........124 


\section{List of Tables}

Table 1.1. Electron Affinities of Third Row Transition Metal Hexafluorides ${ }^{6}$. .4

Table 3.1. X-ray powder data for $\mathrm{LiPtF}_{6}$ with a rhombohedral unit cell. 25

Table 3.2. Synthesis of $\mathrm{AMF}_{6}$ from $\mathrm{AF}$ and $\mathrm{MF}_{5}$ 27

Table 3.3. X-ray powder data for $\mathrm{KRuF}_{6}$ with a rhombohedral unit cell. .28

Table 3.4. X-ray powder data for $\mathrm{KRhF}_{6}$ with a rhombohedral unit cell. .29

Table 3.5. X-ray powder data for $\mathrm{LiIrF}_{6}$ with a rhombohedral unit cell. .30

Table 4.1. X-ray powder data for $\mathrm{O}_{2} \mathrm{RhF}_{6}$ with a cubic unit cell. .38

Table 5.1. X-ray powder data for $\mathrm{Ag}\left(\mathrm{BiF}_{6}\right)_{2}$ with a triclinic unit cell. .52

Table 5.2. X-ray powder pattern of $\mathrm{AgBiF}_{6}$ with a tetragonal unit cell. 61

Table 6.1. Crystal Data and Details of the Structure Refinement of $\mathrm{AgFBF}_{4}$ .82

Table 6.2. Positional Parameters for $\mathrm{AgFBF}_{4}$ at $293 \mathrm{~K}$ .84

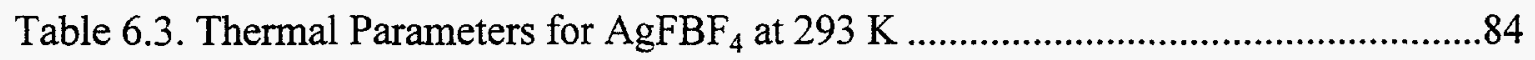

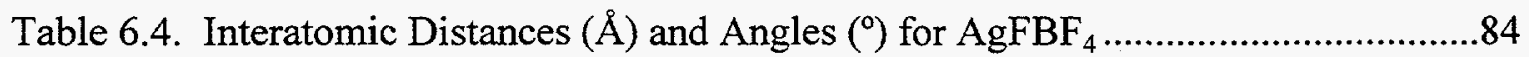

Table 6.5. Crystal Data and Details of the Structure Refinement of $\operatorname{AgFIrF}_{6} \ldots \ldots \ldots \ldots \ldots \ldots . . .87$

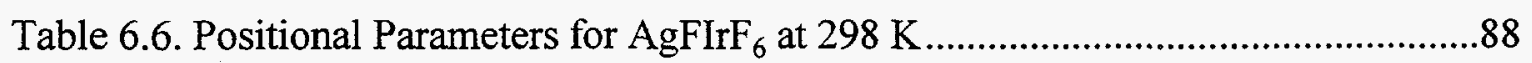

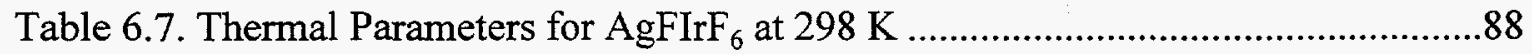

Table 6.8. Interatomic Distances $(\AA)$ and Angles $\left(^{\circ}\right)$ for $\mathrm{AgFIrF}_{6}$ at $298 \mathrm{~K} \ldots \ldots \ldots \ldots \ldots \ldots . . . . . .19$

Table 6.9. Crystal Data and Details of the Structure Refinement of $\mathrm{AgFRuF}_{6} \ldots \ldots \ldots \ldots \ldots . . .93$

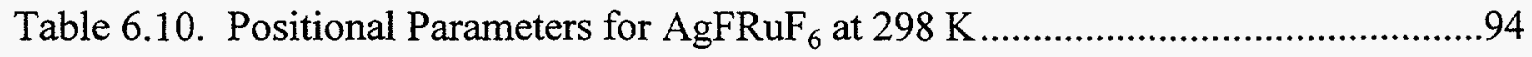

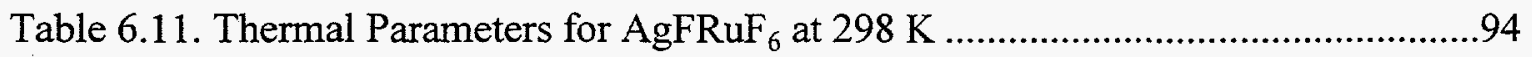


xii

Table 6.12. Interatomic Distances $(\AA)$ and Angles $\left(^{\circ}\right)$ for $\mathrm{AgFRuF}_{6}$ at $298 \mathrm{~K} \ldots \ldots \ldots \ldots . . .95$

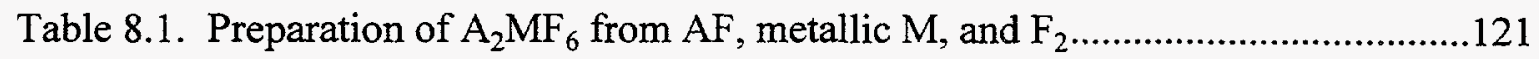




\section{Acknowledgments}

I would like to thank Professor Neil Bartlett for his infectious enthusiasm and for giving me the responsibility to determine the details of my research projects. His direction and guidance are greatly appreciated, as is his readiness to share his knowledge and wisdom.

Bill Casteel deserves the recognition and the blame for training me in the fundamental techniques of fluorine chemistry - thanks, Bill, for your patience and especially for your courage. I am ingratiated to Byron Shen for our many enlightening discussions, and for hosting many parties during his time here. I should express my thanks to the Bartlett Group postdocs, including Horst Borrmann, Rika Hagiwara, Jörg Münzenberg, Philippe Botkovitz, Scott Elder, and Marc Whalen - I learned directly from all of them, and indirectly by sharing my experience with the later ones. Another very special colleague was Professor Boris Žemva, whose diligence and friendly demeanor helped me to get through the tedious and frustrating parts of my work. And thanks to Lisa Chacón for sharing her creativity and vitality. Finally, my friends Carrie, Harry, Dave, Rick, and most of the Stacy Group have brought me many good times and carried me through the difficult times.

This work was supported by the U.S. Department of Energy under contract No. DE-AC03-76SF00098 and supplemented for the first two years by fellowship money provided by the Department of Education. 


\section{Chapter 1}

\section{Introduction and Experimental Considerations}

\subsection{General Introduction}

In 1989 , a synthetic route to thermally unstable fluorides such as $\mathrm{NiF}_{4}, \mathrm{NiF}_{3}$, and $\mathrm{AgF}_{3}$ was discovered. ${ }^{1}$ Because of their instability, attempts to generate these materials with standard high-pressure fluorination reactions had all failed. Dissolution of $\mathrm{AgF}_{3}$ in anhydrous hydrogen fluoride (aHF) solution by acidification with arsenic pentafluoride, in an attempt to prepare a cationic $\mathrm{Ag}(\mathrm{III})$ salt was found, in these laboratories, to generate $\mathrm{AgFAsF}_{6}$ with slow release of elemental fluorine. ${ }^{2}$ Frlec et al. ${ }^{3}$ had first made this $\mathrm{AgF}^{+}$salt by the interaction of the same acidic solution on the divalent silver compound $\mathrm{AgF}_{2}$. In their search for evidence for a cationic $\mathrm{Ag}(\mathrm{III})$ intermediate in the $\mathrm{AgF}_{3}$ reduction in acidified aHF, Bartlett and Žemva and their coworkers ${ }^{2}$ discovered the unexpectedly high electron-withdrawing potential of cationic $\mathrm{Ag}(\mathrm{II})$, which was demonstrated in its oxidation of xenon gas to Xe(II), and, later, in its oxidation ${ }^{4}$ of 
oxygen gas to $\mathrm{O}_{2}^{+}$. These results indicated that cationic $\mathrm{Ag}(\mathrm{II})$ rivaled even platinum hexafluoride in electron affinity. It was soon recognized that such remarkable oxidizing power available in the divalent $\mathrm{Ag}(\mathrm{II})$ solution species presaged potentially unmatched oxidizing power in the (as yet unobserved) trivalent solution species, $\mathrm{Ag}_{\text {solv }}^{\text {III }}$. Experiments were devised to investigate the possibility of generating and utilizing the $\mathrm{Ag}_{\text {solv }}^{\text {III }}$ species in acidic aHF solution before its decomposition into $\mathrm{Ag}_{\text {solv }}^{\text {II }}$ and fluorine gas. Reactions of this design revealed $\mathrm{Ag}_{\text {solv }}^{\text {III }}$ to be a truly remarkable oxidizer even at temperatures below $0^{\circ} \mathrm{C}$.

However, the reactivity of these silver materials was only one of their novel features. Because $\mathrm{Ag}(\mathrm{II})$ is a $d^{q}$ species, distortion from octahedral symmetry is often found, as explained by Crystal Field Theory, as a consequence of the Jahn-Teller effect. Gantar et $a l^{5}$ had solved the crystal structure of $\mathrm{AgFAsF}_{6}$ in 1987, and this had revealed a one-dimensional cation of formula $(\mathrm{AgF})_{\mathrm{n}}^{\mathrm{n}+}$, in which the silver was essentially linearly coordinated with two nearly identical Ag-F bond lengths, five anion F ligands at long interatomic distances $(\sim 2.4 \AA)$ completing the coordination. This approximately $\mathrm{D}_{\infty \mathrm{h}}$ symmetry (with the Ag-F-Ag axis designated $z$ ) requires that the antibonding sigma orbital, $d_{z^{2}}$, be the singly occupied orbital (SOMO). It was in work performed in these laboratories, however, that the magnetic characteristic exhibited by all $\mathrm{AgF}^{+}$salts was discovered: a small temperature-independent paramagnetism evident over a very broad temperature range. Such magnetic behavior can indicate an electronically conductive 
system, which, if present, would likely be one-dimensional in nature, owing to the structure of $\mathrm{AgF}^{+}$salts. By analogy with the high oxidation state copper oxide systems, the strong oxidizing properties of $\mathrm{AgF}^{+}$raised the possibility of superconductivity. Additionally, the structures of other $(\mathrm{AgF})_{\mathrm{n}}^{\mathrm{n}+}$ salts with different anions remained to be explored. In many $\mathrm{Cu}(\mathrm{II})$ systems, a Jahn-Teller distortion results in elongation along one axis of the octahedron, yielding a square-coordinated metal center. Such a structural feature seemed probable in $\mathrm{AgF}_{2}^{+}$systems, if they could be prepared, and possible in the $\mathrm{Ag}(\mathrm{II})$ system as well. More likely than the latter were mixed oxidation state $\mathrm{Ag}(\mathrm{III}) \mathrm{Ag}(\mathrm{II})$ materials containing $\left(\mathrm{AgF}_{2}\right)_{\mathrm{n}}^{+}$sheets with a two-dimensional network more closely related to those observed in $\mathrm{Cu}(\mathrm{II})$, so-called pseudo-perovskite materials.

\subsection{Electron Affinities of Third Row Transition Metal Hexafluorides}

For a more quantitative investigation of the oxidizing power of silver salts, it was desirable to have a scale against which potentials could be quantitatively measured. Due to the extremely reactive nature of the systems under investigation and their recent discovery, electrochemical techniques have yet to be devised. However, the values of the electron affinities of third row transition metals (from tungsten to gold) span the observed redox range needed for these silver systems, and do so with an essentially linear dependence of affinity on the atomic number of the metal atom involved (see Figure 1.1). Thus, release of neutral hexafluoride from these third row metal hexafluoridemonoanions serves as a semi-quantitative measure of the oxidizing potential of the 
species capable of releasing one of the $\mathrm{MF}_{6}$ but not the next higher in the series. For example, a silver species which can liberate gaseous $\mathrm{OsF}_{6}$ from the dissolved $\mathrm{OsF}_{6}^{-}$but not the $\mathrm{IrF}_{6}$ from $\mathrm{IrF}_{6}^{-}$probably has an oxidizing potential of at least $130 \mathrm{kcal} \cdot \mathrm{mol}^{-1}$, but is unlikely to exceed $155 \mathrm{kcal} \cdot \mathrm{mol}^{-1}$ in that potential.

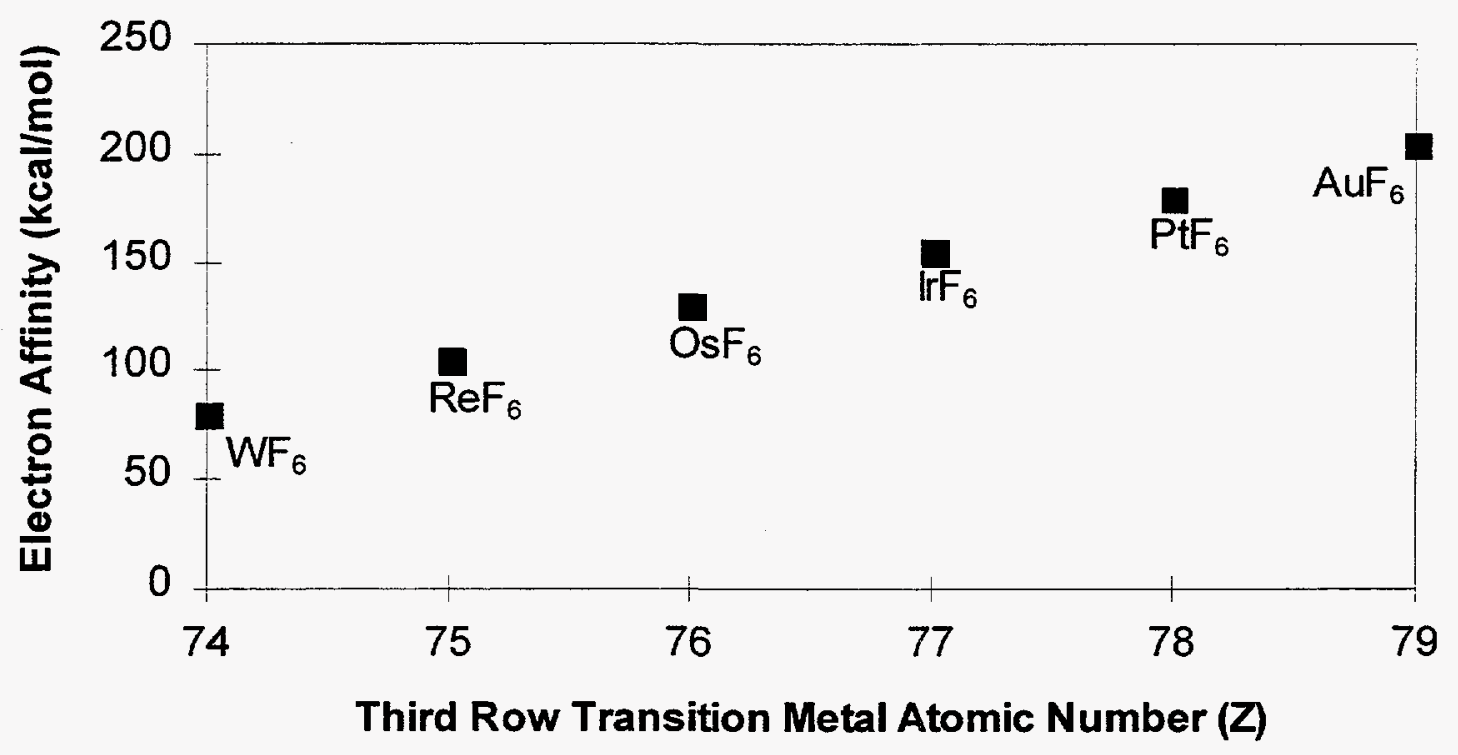

Figure 1.1. Electron Affinities of Third Row Transition Metal Hexafluorides. ${ }^{6}$

Table 1.1. Electron Affinities of Third Row Transition Metal Hexafluorides ${ }^{6}$ $\left(\mathrm{kcal} \cdot \mathrm{mol}^{-1}\right)$

\begin{tabular}{|c|c|c|c|c|c|}
\hline $\mathrm{WF}_{6}$ & $\mathrm{ReF}_{6}$ & $\mathrm{OsF}_{6}$ & $\mathrm{IrF}_{6}$ & $\mathrm{PtF}_{6}$ & AuF $_{6}{ }^{\mathrm{a}}$ \\
\hline 81 & 102 & 133 & 155 & 184 & 205 \\
\hline
\end{tabular}

${ }^{a} \mathrm{AuF}_{6}$ is as yet an unknown compound; the electron affinity is a calculated estimate. 


\subsection{Relative Fluoride Ion Affinities of Some Fluoro-acids}

The work presented herein provides excellent examples of the importance of Lewis acid-base considerations in synthetic inorganic chemistry. In the aHF solvent system, a Lewis acid behaves as a fluoride ion acceptor and a Lewis base as a fluoride ion donor. In order to effectively predict the outcome of a reaction, it is critical to know the relative fluoride ion affinities (a.k.a., fluoro-acidities) of all species involved. This significance is illustrated well in the $\mathrm{Ag}(\mathrm{III})$ reactions of Chapter 4. Here, it is seen that $\mathrm{KAgF}_{4}$ is stable in aHF solution,

$$
\mathrm{KAgF}_{4(\mathrm{~s})} \stackrel{\mathrm{aHF}}{\rightleftharpoons} \mathrm{K}_{(\text {solv })}^{+}+\mathrm{AgF}_{4(\text { solv })}^{-}
$$

thus indicating that $\mathrm{AgF}_{3}$ is a stronger fluoro-acid than is $\mathrm{HF}$ itself. By contrast, such is probably not the case with $\mathrm{CuF}_{3}$, as it appears that $\mathrm{K}_{3} \mathrm{CuF}_{6}$ generates $\mathrm{CuF}_{3}$ immediately

$$
\mathrm{K}_{3} \mathrm{CuF}_{6(s)} \stackrel{\mathrm{aHF}}{\longrightarrow} 3 \mathrm{~K}_{(\text {solv })}^{+}+3\left(\mathrm{HF}_{\mathrm{n}} \mathrm{F}_{(\text {solv })}^{-}+\mathrm{CuF}_{3(\mathrm{~s})}\right.
$$

upon exposure to liquid aHF. Addition of a very weak fluoro-acid (e.g. $\left.\mathrm{SiF}_{4}\right)$ to the $\mathrm{KAgF}_{4}$ solution results in no reaction:

$$
\mathrm{KAgF}_{4(s)} \stackrel{\mathrm{aHF}}{\underset{\mathrm{SiF}_{4}}{\rightleftharpoons}} \mathrm{K}_{(\text {solv })}^{+}+\mathrm{AgF}_{4 \text { (solv) }}^{-}
$$

Removal of solvent and acid merely recovers the $\mathrm{KAgF}_{4}$ quantitatively. Introduction of the slightly stronger Lewis acid $\mathrm{BF}_{3}$ strips the fluoride ion off the $\mathrm{AgF}_{4}^{-}$, forming $\mathrm{AgF}_{3}$ and $\mathrm{BF}_{4}^{-}$:

$$
\mathrm{KAgF}_{4(\mathrm{~s})} \underset{\mathrm{BF}_{3}}{\stackrel{\mathrm{aHF}}{\longrightarrow}} \mathrm{K}_{(\text {solv })}^{+}+\mathrm{BF}_{4(\text { solv })}^{-}+\mathrm{AgF}_{3(\mathrm{~s})}
$$


Excess $\mathrm{BF}_{3}$ in this system produces no further reaction.

$$
\mathrm{AgF}_{3(s)} \underset{\mathrm{BF}_{3}}{\stackrel{\mathrm{aHF}}{\longrightarrow}} \mathrm{AgF}_{3(\mathrm{~s})} \quad \text { (no reaction) }
$$

Now, by acidifying the aHF with a still stronger fluoride ion acceptor such as $\mathrm{AsF}_{5}$, the reaction proceeds with the removal of at least one fluoride from the insoluble $\mathrm{AgF}_{3}$, generating the soluble $\mathrm{Ag}_{\text {solv }}^{\text {III }}$ species.

$$
\mathrm{AgF}_{3(s)} \underset{\mathrm{AsF}_{5}}{\stackrel{\mathrm{aHF}}{\longrightarrow}} \mathrm{Ag}_{\text {(solv) }}^{\mathrm{III}}+\mathrm{x} \mathrm{AsF}_{6 \text { (solv) }}^{-}
$$

Thus, selection of the proper fluoro-acid is the key to the preparation of pure $\mathrm{KAgF}_{4}$, pure $\mathrm{AgF}_{3}$, and the strongly oxidizing cationic $\mathrm{Ag}(\mathrm{III})$ system.

A classification of relative fluoride ion affinities in the aHF solvent system may be roughly determined through reaction chemistry. A series of acid-displacement reactions spanning a wide range of fluoro-acidities is useful for identification of appropriate reagents in synthetic fluorine chemistry. While factors such as lattice energy, solvation enthalpy, and change in entropy are not considered here, the hierarchical ranking obtained should have practical utility for the synthetic fluorine chemist.

\subsubsection{Fluoro-acidity Ranking of Main Group Fluorides}

Main group fluorides are frequently used as Lewis acid reagents and catalysts, many of them generating superacid ${ }^{8}$ conditions in HF solution. HF itself autoionizes according to Equation (7). ${ }^{8}$

$$
2 \mathrm{HF} \rightleftharpoons \mathrm{H}_{2} \mathrm{~F}^{+}+\mathrm{F}^{-} \quad \mathrm{K} \sim 10^{-10}
$$


The Group I fluorides ( $\mathrm{LiF}$ through $\mathrm{CsF}$ ) are all very good fluoro-bases in this solvent. Group II fluorides are also basic but of low solubility in aHF. Boron and aluminum are the only Group III elements whose fluorides are commonly used for their ability to act as Lewis acids. Boron trifluoride has a high fluoride ion affinity; the molecule is planar in the gas phase and becomes tetrahedral upon formation of the conjugate base $\mathrm{BF}_{4}^{-}$. Equations (1) and (4) above suggest that $\mathrm{BF}_{3}$ has a significantly higher fluoride ion affinity than $\mathrm{HF}$. In the solid $\mathrm{AlF}_{3}$, the aluminum occupies octahedral-hole sites in the $\mathrm{F}$ ligand array. Because of its three-dimensional, energetically favorable structure, $\mathrm{AlF}_{3}$ monomer (even aHF solvated) is not a significantly available species and $\mathrm{AlF}_{3}$ is only a very weak fluoro-acid.

While four-coordinate carbon exhibits fluoro-neutrality as the tetrafluoride, the other Group IV elements may form five-coordinate $\mathrm{MF}_{5}^{-}$and doubly-negative octahedral species. Silicon tetrafluoride is such a poor Lewis acid that, in dilute aHF solution, it does not form fluorosilicates with even the alkali fluoride bases. This failure arises because $\mathrm{HF}$ itself has a higher fluoride ion affinity than $\mathrm{SiF}_{4}$, as can be observed in their reaction chemistry with the strong fluoro-base $\mathrm{KF} . \mathrm{K}_{2} \mathrm{SiF}_{6}$ is not preparable in dilute aHF solution; $\mathrm{KHF}_{2}$ is produced instead.

$$
\mathrm{KF} \underset{\mathrm{SiF}_{4}}{\stackrel{\mathrm{aHF}}{\longrightarrow}} \mathrm{KHF}_{2}
$$

(When the KF concentration is sufficiently high that $\mathrm{F}^{-}$is available over and above that needed to make at least $\mathrm{HF}_{2}^{-}$, then $\mathrm{SiF}_{6}^{2-}$ salt formation can occur.) $\mathrm{K}_{2} \mathrm{SiF}_{6}$ may be 
prepared in water, however, by passing $\mathrm{SiF}_{4}$ into a concentrated aqueous solution of $\mathrm{KF}$. Material obtained in this manner gives an X-ray powder pattern (XRPP) indicative of pure $\mathrm{K}_{2} \mathrm{SiF}_{6}\left(\right.$ cubic $^{9}, \mathrm{a}_{\mathrm{o}}=8.133 \AA$ ). Subsequent dissolution of this product in an abundance of aHF results in rapid evolution of $\mathrm{SiF}_{4}$ and formation of solvated $\mathrm{KF}$, which, upon removal of the solvent, crystallizes from solution as $\mathrm{KHF}_{2}$.

$$
\mathrm{K}_{2} \mathrm{SiF}_{6} \stackrel{\mathrm{aHF}}{\longrightarrow} 2 \mathrm{~K}_{(\text {(solv })}^{+}+2 \mathrm{~F}_{(\text {solv })}^{-}+\mathrm{SiF}_{4(\mathrm{~g})}
$$

Mallouk et al. ${ }^{10}$ have found that $\mathrm{GeF}_{4}$ has only a slightly higher first fluoride ion affinity than that of $\mathrm{BF}_{3}$ (however, the doubly negative ion provides for high lattice enthalpy or solvation enthalpy, which renders $\mathrm{GeF}_{4}$ a rather strong flouro-acid in aHF).

All of the Group V elements except for nitrogen form pentafluorides which span a wide range of fluoride ion affinities. $\mathrm{PF}_{5}$ is the weakest acid in the group, ranking slightly behind the trifluoride of boron. Similar to $\mathrm{BF}_{3}, \mathrm{PF}_{5}$ is seen to generate $\mathrm{AgF}_{3}$ from a solution of $\mathrm{KAgF}_{4}$. However, Žemva et al. ${ }^{11}$ have observed that removal of the $\mathrm{PF}_{5}$ overpressure causes the reaction to go in the reverse direction, thus reforming $\mathrm{KAgF}_{4}$.

$$
\mathrm{K}_{\text {(solv) }}^{+}+\mathrm{AgF}_{4(\text { solv })}^{-} \stackrel{\mathrm{aHF}}{\underset{\mathrm{PF}_{5}}{\rightleftharpoons}} \mathrm{KPF}_{6(\mathrm{~s})}+\mathrm{AgF}_{3(\mathrm{~s})}
$$

This reversibility occurs despite the lower solubility of $\mathrm{KPF}_{6}$ than $\mathrm{KBF}_{4}$ in aHF. Gillespie and coworkers ${ }^{12}$ studied the complete set of Group V pentafluorides and found their hierarchy of acidity to be as follows: $\mathrm{PF}_{5}<\mathrm{AsF}_{5}<\mathrm{BiF}_{5}<\mathrm{SbF}_{5}$. These results are in good agreement with the observations made in this laboratory. 
Overall, the hierarchy of the main group Lewis acids discussed above is as follows, in order of increasing fluoro-acidity:

$$
\mathrm{SiF}_{4}<\mathrm{HF}<\mathrm{PF}_{5}<\mathrm{BF}_{3}<\mathrm{GeF}_{4}<\mathrm{AsF}_{5}<\mathrm{BiF}_{5}<\mathrm{SbF}_{5}
$$

\subsection{Stabilization of High Oxidation State Cations Such as $\mathbf{A g F}_{2}^{+}$}

If cations such as $\mathrm{AgF}_{2}^{+}$are to be stabilized, it is clear that the counter anion must resist both electron transfer and fluoride ion transfer. Although the main group fluoroanions are probably all of sufficiently high ionization potential to resist electron transfer, it is probable that only the poorest $\mathrm{F}^{-}$donors, $\mathrm{SbF}_{6}^{-}$and $\mathrm{BiF}_{6}^{-}$, could resist fluoride transfer to such an electrophilic center as $\mathrm{AgF}_{2}^{+}$. Perhaps the best choice of transition metal counter ion would be the $\mathrm{Au}(\mathrm{V})$ fluoro-anion $\mathrm{AuF}_{6}^{-}$. It was observed in this laboratory that the interaction of $\mathrm{XeF}_{5} \mathrm{AuF}_{6}$ with excess $\mathrm{SbF}_{5}$ in aHF does not result in the displacement of $\mathrm{AuF}_{5}$. Although the product of this reaction is unidentified, it is believed that a complex anion $\mathrm{AuSbF}_{11}^{-}$results.

$$
\mathrm{XeF}_{5} \mathrm{AuF}_{6}+\mathrm{xs} \mathrm{SbF}_{5} \underset{?}{\stackrel{\mathrm{aHF}}{\longrightarrow}} \mathrm{XeF}_{5} \mathrm{AuSbF}_{11}
$$

Such reactivity indicates that $\mathrm{AuF}_{5}$ is comparable to $\mathrm{SbF}_{5}$ in fluoro-acidity, so $\mathrm{AuF}_{6}^{-}$is therefore unlikely to undergo fluoride transfer. And it is improbable that $\mathrm{AuF}_{6}^{-}$would experience electron transfer since the neutral hexafluoride of gold has yet to be observed. These stability considerations apply not only to $\mathrm{AgF}_{2}^{+}$, but to other high oxidation state cationic species at the limits of chemical oxidizability as well. 


\subsection{References}

${ }^{1}$ B. Žemva, K. Lutar, A. Jesih, W. J. Casteel, Jr., and N. Bartlett, J. Chem. Soc., Chem. Comm., (1989), p 346.

${ }^{2}$ B. Žemva, R. Hagiwara, W. J. Casteel, Jr., K. Lutar, A. Jesih, and N. Bartlett, J. Am. Chem. Soc., 112 (1990), pp 4846-4849.

${ }^{3}$ B. Frlec, D. Gantar, and J. H. Holloway, J. Fluorine Chem., 20 (1982), pp 385-396.

${ }^{4}$ W. J. Casteel, Jr., Ph. D. Thesis, (1992) Chapter 7, U.C. Berkeley.

${ }^{5}$ D. Gantar, B. Frlec, D. R. Russell, and J. H. Holloway, Acta Cryst., C43 (1987), pp 618-620.

${ }^{6}$ For WF $:$ P. M. George and J. L. Beauchamp, Chem. Phys., 36 (1979), p 345.

For $\mathrm{ReF}_{6}, \mathrm{OsF}_{6}$, and $\mathrm{IrF}_{6}: \mathrm{N}$. Bartlett, Proc. Of the Robert A. Welch Foundation Conference on Chem. Res., Oct-Nov (1988), p 269.

For $\mathrm{PtF}_{6}$ and $\mathrm{AuF}_{6}:$ M. I. Nikitin, L. N. Sodorov, and M. V. Korobov, Int. J. Mass Spec. Ion Phys., 37 (1981), p 13.

${ }^{7} \mathrm{~K}_{3} \mathrm{CuF}_{6}$ generated in a high-temperature, high-pressure fluorine bomb reactor, when placed into aHF solution, immediately undergoes solvolysis to form the red $\mathrm{CuF}_{3}$ precipitate; unpublished work in this group, J. Münzenberg, 1993.

${ }^{8}$ F. A. Cotton and G. Wilkinson, Advanced Inorganic Chemistry, 5th ed. (1988), New York: John Wiley and Sons. 
${ }^{9}$ Natl. Bur. Stand. (U.S.), Circ. 539, 5 (1955), p 50.

${ }^{10}$ T. E. Mallouk, G. L. Rosenthal, G. Müller, R. Brusasco, and N. Bartlett, Inorg. Chem., 23 (1984), pp 3167-3173.

${ }^{11}$ B. Žemva, K. Lutar, A. Jesih, W. J. Casteel, Jr., A. P. Wilkinson, D. E. Cox, R. B. Von Dreele, H. Borrmann, and N. Bartlett, J. Am. Chem. Soc., 113 (1991), pp 4192-4198.

${ }^{12}$ R. J. Gillespie, K. Ouchi, G. P. Pez, Inorg. Chem., 8 (1969), p 63. 


\section{Chapter 2}

\section{Experimental Methods}

\subsection{Apparatus}

A stainless steel and monel vacuum line fitted with a Helicoid Instruments

(Watertown, CT) pressure gauge (fluorine service, 1500 torr) and rotary vane mechanical pump was used for the handling of gases. Autoclave Engineers (Erie, PA) supplied the stainless steel and monel valves (series $30 \mathrm{VM}$ ). A fluorine gas supply was connected to a high-pressure (500 psi, $3.8 \times 10^{5}$ torr) fluorine service Helicoid gauge and then to the vacuum line. Low pressure measurements were made with the use of a thermocouple gauge head (Varian Vacuum Products, Santa Clara, CA; model 0531) which was read by a millitorr gauge (Varian, model 801, 0 - 2 torr). For reactions involving the use of aHF, a section of vacuum line constructed from FEP tubing (Chemplast, Inc., Wayne, NJ) and Teflon valves, as described elsewhere ${ }^{1}$, and unions (Oakland Valve and Fitting Co., Concord, CA) was attached to the metal line. Reactors for aHF solvent reactions were made from two FEP tubes (diameter $3 / 8$ " or $1 / 2$ ", length $\sim 20 \mathrm{~cm}$ ) which had been sealed 
at one end by heating over a Bunsen flame and then clamping shut with pliers. These two tubes were joined at right angles via Teflon Swagelok compression fittings to a Teflon $\mathrm{T}$ (Oakland Valve and Fitting Co.), the assembly being connected via FEP tubing to a Teflon valve (see Figure 2.1). Volatile fluoride compounds were destroyed by passing through a copper column (diameter $5 \mathrm{~cm}$, length $30 \mathrm{~cm}$ ) containing granular soda lime. Solid fluorides were handled inside a Vacuum Atmospheres (Hawthorne, CA) DRILAB containing a dry argon atmosphere.

\subsection{Characterization Techniques}

\subsubsection{X-ray Powder Diffraction}

When necessary, powder samples were ground in an agate mortar and pestle inside the DRILAB. Quartz capillaries (either $0.3 \mathrm{~mm}$ or $0.5 \mathrm{~mm}$ diameter) were

prepared by heating to $\sim 700^{\circ} \mathrm{C}$ for more than 15 hours under high vacuum (ca. $10^{-8}$ torr). An Enraf Nonius FR 590 X-ray generator fitted with a copper target tube (energized to 40 $\mathrm{kV}$ and $20 \mathrm{~mA}$ ), and a nickel filter provided the required radiation. Typical exposure time was 12 hours with a General Electric Debye-Scherrer cassette loaded with Kodak Industrex AA film (cut to size). X-ray powder patterns (XRPP's) were measured with the aid of a Norelco film-measuring light box. Microsoft Excel for Windows v. 4.0 and v. 5.0, U-fit v. 1.2, and Lazy Pulverix were used in the determination and refinement of unit cell dimensions. 


\subsubsection{Single Crystal X-ray Diffraction}

Because of the highly reactive nature of the materials under study, neither oil nor glue mounts could be used to fix a single crystal. Instead, each crystal was loaded into a quartz capillary, which had been drawn down from $0.3 \mathrm{~mm}$ diameter (to provide a gradual taper) and dried as described above for powder samples. Crystals were gently tapped into the tapered end of the capillary, sometimes only tenuously holding their position. Data was collected on an Enraf Nonius Diffractis 585 diffractometer fitted with a graphite monochromator and a molybdenum target tube energized to $50 \mathrm{kV}$ and $26 \mathrm{~mA}$.

\subsubsection{SQUID Magnetometry}

A Quantum Design (San Diego, CA) SQUID magnetometer (model MPMS) was used to take magnetic measurements on powder samples. The sample container was constructed from two Kel-F cylindrical cups, one fitting inside the other to provide an internal volume $6.5 \mathrm{~mm}$ in diameter and $6.5 \mathrm{~mm}$ in length. A known quantity of powder material was loaded into the smaller cup and was capped by the larger, each having been passivated with 1500 torr $F_{2}$. A thin ring of halocarbon grease around the outside of the smaller cup ensured a gas-tight seal. The two cups were tied together with nylon line.

\subsection{Anhydrous HF as an Oxidatively Inert Solvent}

Traditionally, high oxidation state fluorine chemistry has required the use of high pressures of molecular fluorine heated to hundreds of degrees in a metal "bomb" reactor. While this preparative technique is still quite useful and even necessary at times, the use of a liquid solvent at or below room temperature is preferred for its safety with respect to 
fires and explosions. Although there are many fluorinated solvents which can be used with highly oxidizing materials, none seems to offer the convenience of handling that anhydrous hydrofluoric acid (aHF) does. Although it is highly corrosive and toxic, special handling procedures have been developed which allow for the manipulation of aHF in FEP or Teflon apparatus (sealed from exposure to the air) in much the same way that water is handled in glass. At $19.5^{\circ} \mathrm{C}$, aHF has a vapor pressure $e^{2}$ of $1 \mathrm{~atm}$, so a reactor containing this solvent at room temperature poses no overpressure danger. A colorless liquid, its density is $1.0015 \mathrm{~g} \cdot \mathrm{cc}^{-1}$ at $0^{\circ} \mathrm{C}$, and its dielectric constant is very similar to that of water $\left\{\varepsilon\left(\mathrm{HF}, 0{ }^{\circ} \mathrm{C}\right)=83.6, \varepsilon\left(\mathrm{H}_{2} \mathrm{O}, 0{ }^{\circ} \mathrm{C}\right)=87.7\right\}$. Despite the nonwetting properties of FEP and Teflon, aHF in reactors made of these materials flows much like water in glass; this is because aHF has a much lower surface tension than does water $\left\{\sigma\left(\mathrm{HF}, 0^{\circ} \mathrm{C}\right)=10.1 \mathrm{dyn} \cdot \mathrm{cm}^{-1}, \sigma\left(\mathrm{H}_{2} \mathrm{O}, 18^{\circ} \mathrm{C}\right)=73.1 \mathrm{dyn} \cdot \mathrm{cm}^{-1}\right\}^{2,3}$.

Another feature of aHF that makes it a good solvent for the study of high oxidation state fluorides is its oxidative inertness. The systems under study and discussed here are among the most oxidizing known. Solvents which might traditionally be considered stable to oxidation, such as carbon tetrachloride or acetonitrile, will burn vigorously in contact with many of the materials under investigation. Unfortunately, because of the acidity of aHF, contact with metal vacuum line apparatus must be carefully avoided when a reaction is in progress in order to prevent contamination by hydrogen gas. Furthermore, glass apparatus must be protected from any contact with 
aHF in order to prevent severe etching. Such etching is, in fact, a problem which arises with the X-ray capillary samples if the powder inside the quartz capillary has not been thoroughly dried or contains solvated HF. Although dry solid fluorides are usually kinetically stable in contact with quartz, chemical attack of the capillary wall by $\mathrm{HF}$ generates $\mathrm{H}_{2} \mathrm{O}$, which can then react with the fluoride sample to reform the $\mathrm{HF}$ in a catalytically destructive cycle.

\subsection{Reaction Technique}

Construction of a typical reactor for use with high-oxidation state chemistry in aHF solution is described above and is depicted in Figure 2.1. The Swagelok fittings which connect the FEP tubing to the Teflon tee permit the safe pressurization of the reactor to three atmospheres absolute pressure. All reactors are assembled outside the DRILAB, evacuated, then filled with 2 atm $F_{2}$ gas and left to sit for several hours. After removal of this fluorine to a soda lime scrubber under dynamic vacuum, the reactor is brought into the DRILAB where the Swagelok connections allow for removal of the reactor tubes so that they may be loaded with solid reagents. 


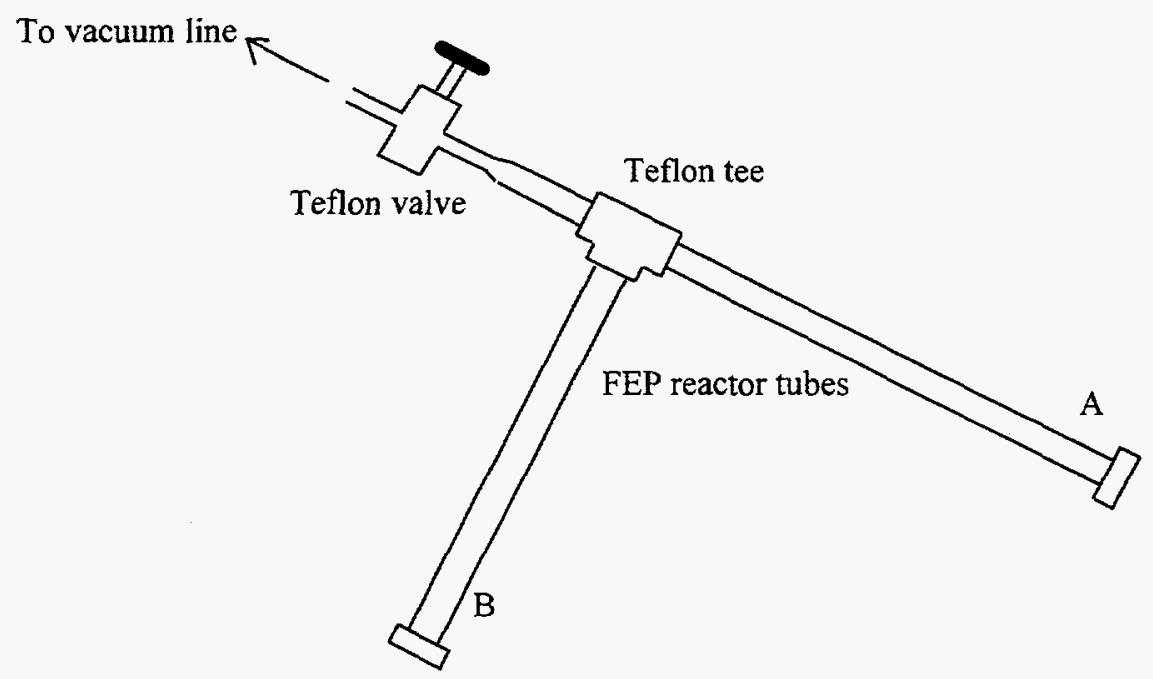

Figure 2.1. A typical reactor tube for use with anhydrous HF solution chemistry.

After removing the reactor from the DRILAB and connecting to the vacuum line, the Teflon valve is opened to the vacuum and the argon atmosphere is evacuated. Usually, the reactor tubes are loaded so that the reaction takes place in tube A. By cooling one or both of the FEP tubes in liquid nitrogen $\left(\mathrm{LN}_{2}\right)$, aHF is easily condensed in from a reservoir tube. Thermal control of the reaction is maintained by immersing the reaction tube in a cold-bath (reactions requiring temperatures above $25^{\circ} \mathrm{C}$ have not been attempted because of the danger of reactor overpressurization). Gases are tensimetrically admitted through delicate control of the Teflon valve. Reaction mixing is performed with the aid of either a Teflon-coated magnetic stir bar placed inside the reactor tube, or by mechanical tapping of the tube from an external device. Separations based upon solubility in the aHF are accomplished by careful decantation of the solution from one arm of the reactor to the other. Following a decantation, the aHF can be condensed back 
over using a cold-bath. The aHF solvent and any remaining gaseous reagents or undesired gaseous products are removed by slow evaporation under dynamic vacuum with continuous agitation in order to reduce violent boiling of the aHF. 


\subsection{References}

${ }^{1}$ K. Lutar, A. Jesih, I. Leban, B. Žemva, and N. Bartlett, Inorg. Chem., 28 (1989), p 3467.

${ }^{2}$ Matheson Gas Data Book, 4th edition (1966), New Jersey: The Matheson Company, Inc.

${ }^{3}$ R. C. Weast, ed., CRC Handbook of Chemistry and Physics, 70th edition (1989), Florida: CRC Press, Inc.. 


\section{Chapter 3}

\section{Preparation of Reagent Materials}

\subsection{Introduction}

Despite the existence of several commercial suppliers of good quality fluorine compounds, the study of high oxidation state fluorine chemistry requires the synthesis of many rudimentary materials that are not commercially available. This deficiency in availability is due, in part, to the very low demand of rarely used compounds which may also contain expensive transition metals, as well as to the great effort and expense required to maintain high oxidation state compounds in storage. Syntheses for main group binary fluorides and ternary alkali fluorides have generally been known for many years. However, older techniques frequently involve the use of high temperatures and high pressures of fluorine. With the advent of Teflon and translucent FEP, the easy manipulation of aHF in a non-corroding container has permitted the development of techniques which utilize this liquid as a solvent at room temperature and at pressures very close to atmospheric. Such reactions are inherently safer and usually lead to greater 
yields and cleaner products. For this reason, synthetic procedures involving these newer aHF solvent techniques (which have become routine in this laboratory) to generate new, as well as previously-described, materials in a novel manner are described here.

\subsection{Commercially Available Reagents}

Anhydrous HF (aHF) (Matheson, Newark, CA) was condensed from the cylinder into a reservoir FEP tube containing $\mathrm{K}_{2} \mathrm{NiF}_{6}$ (Ozark-Mahoning Pennwalt, Tulsa, $\mathrm{OK}$ ) in order to destroy trace quantities of water. Fluorine gas (Matheson) was used as received. $\mathrm{BF}_{3}, \mathrm{PF}_{5}$ (Matheson), and $\mathrm{AsF}_{5}$ (Ozark-Mahoning) gases were checked by I.R. spectroscopy to ensure absence of major impurities, then used as supplied. Liquid $\mathrm{SbF}_{5}$ (Ozark-Mahoning) was distilled from its cylinder into a reservoir FEP tube and stored in the DRILAB. Solid $\mathrm{BiF}_{5}$ (Ozark-Mahoning) was recrystallized from aHF solution prior to use in an attempt to free it of any $\mathrm{BiF}_{3}$ that might be present. $\mathrm{AgF}$ is light sensitive, thus necessitating its recrystallization from aHF. However, because $\mathrm{AgF}$ forms a fairly stable bifluoride, prolonged evacuation at room temperature was required in order to carefully remove all complexed $\mathrm{HF}$ in the recrystallization product. $\mathrm{LiF}, \mathrm{NaF}$, and $\mathrm{KF}$ were evacuated overnight to $10^{-8}$ torr prior to storage in the DRILAB. Transition metals were reduced with a flowing hydrogen atmosphere in a platinum crucible over a flame prior to storage in the DRILAB.

\subsection{Preparation of $\operatorname{IrF}_{5}$ and $\operatorname{IrF}_{6}$}

In the DRILAB, approximately $2.5 \mathrm{~g}(\sim 13 \mathrm{mmol})$ reduced Ir metal was placed into a passivated monel reaction vessel having a Teflon gasket seal. After evacuating the 
can reactor, $\mathrm{F}_{2}$ gas $(\sim 13 \mathrm{mmol})$ was condensed in at $-196^{\circ} \mathrm{C}$. A forced-air heat gun was used to heat the can for ca. $10 \mathrm{~min}$, after which the can was cooled to room temperature and opened to the line. The pressure of fluorine that remained equated to about onefourth the quantity that had been introduced. Again, the can was cooled to $-196{ }^{\circ} \mathrm{C}$ and $13 \mathrm{mmol} \mathrm{F}_{2}$ gas condensed in. This was heated again with the heat gun for $10 \mathrm{~min}$, then cooled back down to $-196^{\circ} \mathrm{C}$. Another $15 \mathrm{mmol} \mathrm{F}$ gas was condensed into the reactor can, and it was heated to $225^{\circ} \mathrm{C}$ overnight in a sand bath. The following day, another 20 mmol $\mathrm{F}_{2}$ gas was introduced at $-196^{\circ} \mathrm{C}$, after which the reactor was heated to $245^{\circ} \mathrm{C}$ for ca. $4 \mathrm{~h}$. Cooling the can once again to $-196^{\circ} \mathrm{C}$ permitted removal of the remaining $F_{2}$ under dynamic vacuum without evacuating the $\mathrm{IrF}_{6}$ product.

$\mathrm{IrF}_{6}$ was easily obtained in aliquots from this storage can by vacuum distillation. Once all the $\mathrm{IrF}_{6}$ had been removed and used for reaction chemistry, the can was brought into the DRILAB. Removal of the screw-top lid revealed a copious quantity of bright yellow solid whose XRPP proved to be that of pure $\operatorname{IrF}_{5}$ \{monoclinic ${ }^{1}, a_{0}=12.267(3), b_{0}$ $\left.=9.982(4), c_{0}=5.431(2) \AA, \beta=99.9(2)^{\circ}\right\}$. This product was placed into a passivated FEP tube for storage.

\subsection{Preparation of $\mathrm{SF}_{3}^{+} \mathrm{OsF}_{6}^{-}$and $\mathrm{SF}_{3}^{+} \mathrm{IrF}_{6}^{-}$}

Because of the volatility of $\mathrm{OsF}_{6}$ and $\mathrm{IrF}_{6}$ and their reactivity with Teflon and FEP over extended periods of time (evidence for reactivity of $\mathrm{IrF}_{6}$ with $\mathrm{FEP}$ appears within a few hours), initial weights of these two reagents as obtained from their monel storage 
cans are imprecise. Nonetheless, the interaction between the metal hexafluoride dissolved in aHF and $\mathrm{SF}_{4}$ gas \{see Equation (1)\}appears to go to completion as the

$$
3 \mathrm{SF}_{4(\mathrm{~g})}+2 \mathrm{MF}_{6(\mathrm{~g})} \stackrel{\mathrm{aHF}}{\longrightarrow} 2 \mathrm{SF}_{3} \mathrm{MF}_{6(\text { solv })}+\mathrm{SF}_{6(\mathrm{~g})}
$$

desired product may be obtained with no volatile color remaining in the reactor $\left(\operatorname{IrF}_{6}\right.$ is a yellow gas and $\mathrm{OsF}_{6}$ is colorless in the gas phase but turns yellow upon condensation). Details of this reaction for the iridium are provided here; all else being equal, osmium hexafluoride requires a greater reaction time than does iridium hexafluoride.

$\mathrm{IrF}_{6}(240 \mathrm{mg}, 0.783 \mathrm{mmol})$ was condensed at $-196^{\circ} \mathrm{C}$ from its monel storage can into a passivated FEP T-reactor. Approximately $2.5 \mathrm{~mL}$ aHF was condensed onto the yellow solid. Upon warming to room temperature, a yellow solution formed above undissolved yellow solid. Introduction of a large excess of $\mathrm{SF}_{4}$ gas resulted in the disappearance of the yellow solid within a few minutes time while intermittently cooling the solution then rewarming it in order to encourage dissolution of the $\mathrm{SF}_{4}$. After $1.5 \mathrm{~h}$, the solution retained a slight yellow tint. The reactor was evacuated, slowly removing the aHF solvent. Because of the extreme solubility of $\mathrm{SF}_{3} \mathrm{IrF}_{6}$ in aHF, no solid precipitated from solution; rather, a light yellow powder crashed out of solution just as the last bit of liquid aHF was removed. An X-ray powder photograph of the product gives only the XRPP of $\mathrm{SF}_{3} \operatorname{IrF}_{6}\left(\right.$ cubic $\left.^{2}, \mathrm{a}_{\mathrm{o}}=5.581(4) \AA\right)$. The weight of the powder $(282 \mathrm{mg})$ suggests a $91 \%$ yield of the desired product based upon the imprecise weight of $\operatorname{IrF}_{6}$ reagent. 


\subsection{Preparation of $\mathrm{AMF}_{6}$ salts}

\subsubsection{Synthesis of $\mathrm{LiPtF}_{6}$ and $\mathrm{KPtF}_{6}$ from $\mathrm{O}_{2}^{+} \mathrm{PtF}_{6}^{-}$}

In two separate reactions, passivated FEP T-reactors were loaded with alkali salt (LiF: $29 \mathrm{mg}, 1.1 \mathrm{mmol} ; \mathrm{KF}: 107 \mathrm{mg}, 1.84 \mathrm{mmol})$ in one tube and $\mathrm{O}_{2} \mathrm{PtF}_{6}(343 \mathrm{mg}, 1.01$ mmol; $354 \mathrm{mg}, 1.04 \mathrm{mmol}$, respectively) in the other. About $2 \mathrm{~mL}$ aHF was condensed into the reactor at $-196^{\circ} \mathrm{C}$, divided equally between the two reagents. The alkali salt solution was poured onto the partially dissolved $\mathrm{O}_{2} \mathrm{PtF}_{6}$, resulting in immediate effervescence. Once the bubbling had slowed, the reactor was opened to release some of the pressure that had built up. Then it was closed again and agitated continuously until no bubbles were seen to form. The potassium salt was sufficiently insoluble in the aHF to permit decantation of the supernatant solution. However, the lithium salt was completely dissolved and could not be washed free of excess LiF. X-ray powder photography confirmed the presence and purity of the two alkali salts (for $\mathrm{LiPtF}_{6}$, see

Table 3.1; for $\mathrm{KPtF}_{6}$, the unit cell is rhombohedral ${ }^{3}, \mathrm{a}_{0}=4.96 \AA, \alpha=97.4^{\circ}$ ). The $\operatorname{LiPtF}_{6}$ was deep orange in color while the $\mathrm{KPtF}_{6}$ was orange-yellow. Yields were essentially quantitative $\left(\mathrm{LiPtF}_{6}: 324 \mathrm{mg}, 102 \% ; \mathrm{KPtF}_{6}: 348 \mathrm{mg}, 96 \%\right)$. 
Table 3.1. X-ray powder data for $\mathrm{LiPtF}_{6}$ with a rhombohedral unit cell. $a_{0}=5.386(5) \AA ; \alpha=55.56(1)^{\circ} ; V=99.09 \AA^{3}$.

\begin{tabular}{|c|c|c|c|c|c|c|c|c|c|c|c|}
\hline \multirow[b]{2}{*}{$\mathrm{I} / \mathrm{I}_{\mathrm{o}}$} & \multicolumn{2}{|c|}{$1 / \mathrm{d}^{2} \times 10^{4}$} & \multirow[b]{2}{*}{$\mathrm{h}$} & \multirow[b]{2}{*}{$\mathrm{k}$} & \multirow[b]{2}{*}{1} & \multirow[b]{2}{*}{$\mathrm{I} / \mathrm{I}_{\mathrm{o}}$} & \multicolumn{2}{|c|}{$1 / d^{2} \times 10^{4}$} & \multirow[b]{2}{*}{$\mathrm{h}$} & \multirow[b]{2}{*}{$\mathrm{k}$} & \multirow[b]{2}{*}{1} \\
\hline & obsd. & calc. & & & & & obsd. & calc. & & & \\
\hline$s$ & 488 & 485 & 1 & 1 & 1 & $\overline{v w b}$ & 8283 & 8284 & 4 & 2 & 0 \\
\hline vs & 586 & 583 & 1 & 0 & 0 & $\mathrm{vvw}$ & 8529 & 8513 & 3 & -1 & -1 \\
\hline $\mathrm{vsb}$ & 749 & 744 & 1 & 1 & 0 & vvw & 8669 & 8674 & 2 & 2 & -2 \\
\hline $\mathrm{s}$ & 1400 & 1391 & 2 & 1 & 1 & vwb & 9108 & 9088 & 5 & 3 & 2 \\
\hline $\mathrm{m}$ & 1595 & 1586 & 1 & 0 & -1 & $\mathrm{vw}$ & 9363 & 9344 & 5 & 4 & 3 \\
\hline $\mathrm{ms}$ & 1884 & 1876 & 2 & 2 & 1 & vw & 9346 & 9344 & 5 & 4 & 3 \\
\hline $\mathrm{s}$ & 2076 & 2071 & 2 & 1 & 0 & vw & 10716 & 10708 & 5 & 3 & 1 \\
\hline mw & 2181 & 2169 & 1 & 1 & -1 & vw & 10713 & 10708 & 5 & 3 & 1 \\
\hline $\mathrm{m}$ & 2339 & 2330 & 2 & 0 & 0 & $\mathrm{w}$ & 10924 & 10907 & 4 & 1 & -1 \\
\hline $\mathrm{ms}$ & 2986 & 2977 & 2 & 2 & 0 & $\mathrm{w}$ & 10920 & 10907 & 4 & 1 & -1 \\
\hline $\mathrm{w}$ & 3179 & 3169 & 3 & 2 & 2 & $w$ & 11108 & 11099 & 5 & 1 & 1 \\
\hline vvw & 3401 & & & & & vw & 11142 & 11099 & 5 & 1 & 1 \\
\hline $\mathrm{w}$ & 3475 & 3462 & 3 & 1 & 1 & & 11142 & 11102 & 3 & -1 & -2 \\
\hline $\mathrm{s}$ & 3537 & 3526 & 3 & 2 & 1 & vw & 12276 & 12261 & 5 & 4 & 1 \\
\hline $\mathrm{m}$ & 3767 & 3755 & 2 & 0 & -1 & $\mathrm{w}$ & 12529 & 12516 & 6 & 3 & 3 \\
\hline $\mathrm{m}$ & 3922 & 3916 & 2 & 1 & -1 & vw & 12529 & 12516 & 6 & 3 & 3 \\
\hline w & 3997 & 3977 & 3 & 3 & 2 & vw & 12682 & 12675 & 6 & 4 & 4 \\
\hline $\mathrm{m}$ & 4576 & 4563 & 3 & 1 & 0 & vvw & 12680 & 12675 & 6 & 4 & 4 \\
\hline \multirow[t]{2}{*}{$\mathrm{mb}$} & 4770 & 4755 & 3 & 3 & 1 & & 12680 & 12685 & 5 & 2 & 0 \\
\hline & 4770 & 4758 & 2 & -1 & -1 & $\mathrm{~m}$ & 13051 & 13042 & 4 & 3 & -1 \\
\hline $\mathrm{mw}$ & 5058 & 5048 & 3 & 2 & 0 & $w$ & 13055 & 13042 & 4 & 3 & -1 \\
\hline $\mathrm{mwb}$ & 5260 & 5243 & 3 & 0 & 0 & $\mathrm{w}$ & 13493 & 13493 & 5 & 3 & 0 \\
\hline wb & 5584 & 5563 & 4 & 2 & 2 & vw & 13506 & 13493 & 5 & 3 & 0 \\
\hline $\mathrm{mb}$ & 5953 & 5950 & 4 & 3 & 2 & $w b$ & 14094 & 14079 & 3 & 3 & -2 \\
\hline \multirow[t]{2}{*}{$\mathrm{mb}$} & 6348 & 6341 & 4 & 2 & 1 & vwb & 14094 & 14079 & 3 & 3 & -2 \\
\hline & 6348 & 6344 & 2 & 0 & -2 & vwb & 14270 & 14261 & 6 & 5 & 3 \\
\hline vwb & 6729 & 6698 & 4 & 1 & 1 & & 14270 & 14274 & 3 & 0 & -3 \\
\hline$w b$ & 6844 & 6829 & 3 & 1 & -1 & vwb & 14757 & 14759 & 4 & 1 & -2 \\
\hline$w b$ & 6954 & 6927 & 2 & 1 & -2 & vvwb & 14769 & 14759 & 4 & 1 & -2 \\
\hline$w b$ & 7098 & 7088 & 3 & 0 & -1 & vw & 15429 & 15433 & 6 & 3 & 1 \\
\hline$w b$ & 7139 & 7149 & 4 & 3 & 1 & $\mathrm{mw}$ & 15465 & 15466 & 6 & 2 & 1 \\
\hline VVw & 7511 & 7502 & 4 & 4 & 2 & vw & 15462 & 15466 & 6 & 2 & 1 \\
\hline \multirow[t]{2}{*}{ vwb } & 7756 & 7735 & 3 & 2 & -1 & vw & 15665 & 15665 & 4 & 2 & -2 \\
\hline & 7756 & 7758 & 4 & 4 & 4 & vvw & 15662 & 15665 & 4 & 2 & -2 \\
\hline $\mathrm{vw}$ & 8224 & 8220 & 4 & 1 & 0 & & & & & & \\
\hline
\end{tabular}




\subsubsection{Preparation of $\mathrm{LiRuF}_{6}$}

Ru powder (161 mg, $1.60 \mathrm{mmol})$ which had been previously reduced in a stream of $\mathrm{H}_{2}$ gas was loaded into one tube of a passivated FEP T-reactor. Into the other tube was placed LiF ( $81 \mathrm{mg}, 3.1 \mathrm{mg})$. After removing the argon from the reactor under vacuum, the two solids were combined. Anhydrous HF ( $2.3 \mathrm{~mL})$ was condensed onto the reagents at $-196^{\circ} \mathrm{C}$ and then warmed to room temperature. All of the $\mathrm{LiF}$ dissolved, but there was no apparent interaction with the Ru powder. The reactor was pressurized to 2 atm with $F_{2}$ gas and continuous agitation was begun. More $F_{2}$ was periodically introduced in order to replace that which was consumed in the reaction. After $16 \mathrm{~h}$ reaction time, the aHF solution appeared orange-brown with a burgundy-red precipitate present. The aHF solution was decanted from the burgundy-red solid. Removal of aHF under dynamic vacuum left a yellow-brown powder. X-ray powder photography of the burgundy material gave only the pattern of $\mathrm{LiRuF}_{6}$ (rhombohedral ${ }^{4}, \mathrm{a}_{0}=5.39 \AA, \alpha=56.0$

$\left.{ }^{\circ}\right)$. No XRPP was taken of the yellow-brown soluble byproduct, which was assumed to be $\mathrm{Li}_{2} \mathrm{RuF}_{6}$. The yield of $\mathrm{LiRuF}_{6}(176 \mathrm{mg})$ was $50 \%$.

\subsubsection{General Synthesis of $\mathrm{AMF}_{6}$ from $A F$ and $M F_{5}$}

Combination of the alkali fluoride and the metal pentafluoride inside a passivated FEP T-reactor was accomplished with a range of alkali salts and pentafluorides. Table 3.2 gives representative reactions for each permutation. All reactions were carried out in 
1.5 to $3 \mathrm{~mL}$ aHF. Separation of pure product from remaining reagents is as described in the final column of the table.

Table 3.2. Synthesis of $\mathrm{AMF}_{6}$ from $\mathrm{AF}$ and $\mathrm{MF}_{5}$

\begin{tabular}{|c|c|c|c|c|}
\hline $\begin{array}{c}\text { Alkali Fluoride } \\
\text { Reagent }\end{array}$ & $\begin{array}{c}\text { Metal } \\
\text { Pentafluoride } \\
\text { Reagent }\end{array}$ & $\begin{array}{c}\text { Product and } \\
\text { Yield }\end{array}$ & $\begin{array}{c}\text { Solubility of } \\
\text { AMF }_{6} \\
\left(a,-20^{\circ} \mathrm{C}\right.\end{array}$ & $\begin{array}{l}\text { Purification } \\
\text { Procedure }\end{array}$ \\
\hline $\begin{array}{c}\text { LiF } \\
29 \mathrm{mg}, 1.1 \mathrm{mmol}\end{array}$ & $\begin{array}{c}\mathrm{AsF}_{5} \\
2 \mathrm{~atm} \text { pressure }\end{array}$ & $\begin{array}{c}\text { LiAsF }_{6} \\
215 \mathrm{mg}, 99 \%\end{array}$ & very high & $\begin{array}{l}\text { Vacuum } \\
\text { removal of } \\
\text { excess } \mathrm{AsF}_{5}\end{array}$ \\
\hline $\begin{array}{c}\mathrm{CsF} \\
153 \mathrm{mg}, 1.01 \\
\mathrm{mmol}\end{array}$ & $\begin{array}{c}\text { AsF }_{5} \\
900 \text { torr pressure }\end{array}$ & $\begin{array}{c}\mathrm{CsAsF}_{6} \\
295 \mathrm{mg}, 91 \%\end{array}$ & $\begin{array}{c}\text { low } \\
(\sim 90 \mathrm{mmol} / \mathrm{l})\end{array}$ & $\begin{array}{c}\text { Vacuum } \\
\text { removal of } \\
\text { excess } \mathrm{AsF}_{5}\end{array}$ \\
\hline $\begin{array}{c}\mathrm{KF} \\
347 \mathrm{mg}, 5.98 \\
\mathrm{mmol}\end{array}$ & $\begin{array}{c}\mathrm{PF}_{5} \\
2 \mathrm{~atm} \text { pressure }\end{array}$ & $\begin{array}{c}\mathrm{KPF}_{6} \\
1090 \mathrm{mg} \\
99 \%\end{array}$ & $\begin{array}{c}\text { low } \\
(\sim 100 \\
\mathrm{mmol} / \mathrm{l})\end{array}$ & $\begin{array}{l}\text { Vacuum } \\
\text { removal of } \\
\text { excess } \mathrm{PF}_{5}\end{array}$ \\
\hline $\begin{array}{c}\mathrm{KF} \\
57 \mathrm{mg}, 0.98 \\
\mathrm{mmol}\end{array}$ & $\begin{array}{c}\mathrm{BiF}_{5} \\
271 \mathrm{mg}, 0.89 \mathrm{mmol}\end{array}$ & $\begin{array}{c}\mathrm{KBiF}_{6} \\
310 \mathrm{mg}, 96 \%\end{array}$ & $\begin{array}{l}\text { very high } \\
(>900 \\
\mathrm{mmol} / \mathrm{l})\end{array}$ & $\begin{array}{l}\text { Decantation of } \\
\text { last drops of } \\
\text { aHF soln. }\end{array}$ \\
\hline $\begin{array}{c}\mathrm{KF} \\
91 \mathrm{mg}, 1.56 \\
\mathrm{mmol}\end{array}$ & $\begin{array}{c}\mathrm{RuF}_{5} \\
214 \mathrm{mg}, 1.09 \mathrm{mmol}\end{array}$ & $\begin{array}{c}\mathrm{KRuF}_{6} \\
239 \mathrm{mg}, 86 \%\end{array}$ & very low & $\begin{array}{c}\text { Decantation and } \\
3 \text { washings in } \\
\text { aHF }\end{array}$ \\
\hline $\begin{array}{c}\mathrm{KF} \\
23 \mathrm{mg}, 0.39 \\
\mathrm{mmol}\end{array}$ & $\begin{array}{c}\mathrm{RhF}_{5} \\
68 \mathrm{mg}, 0.34 \mathrm{mmol}\end{array}$ & $\begin{array}{c}\mathrm{KRhF}_{6} \\
89 \mathrm{mg}, 101 \%\end{array}$ & $\begin{array}{l}\text { unknown } \\
(>160 \\
\text { mmol/l })\end{array}$ & $\begin{array}{c}\text { None } \\
\text { performed }\end{array}$ \\
\hline $\begin{array}{c}\mathrm{LiF} \\
69 \mathrm{mg}, 2.7 \mathrm{mmol}\end{array}$ & $\begin{array}{c}\mathrm{IrF}_{5} \\
749 \mathrm{mg}, 2.61 \mathrm{mmol}\end{array}$ & $\begin{array}{c}\text { LilrF }_{6} \\
369 \mathrm{mg}, 45 \%\end{array}$ & unknown & $\begin{array}{c}\text { None } \\
\text { performed (rxn } \\
\text { incomplete) }\end{array}$ \\
\hline $\begin{array}{c}\mathrm{KF} \\
96 \mathrm{mg}, 1.6 \mathrm{mmol}\end{array}$ & $\begin{array}{c}\mathrm{IrF}_{5} \\
306 \mathrm{mg}, 1.06 \mathrm{mmol}\end{array}$ & $\begin{array}{c}\mathrm{KIrF}_{6} \\
291 \mathrm{mg}, 79 \%\end{array}$ & very low & $\begin{array}{c}\text { Decantation and } \\
1 \text { washing in } \\
\text { aHF }\end{array}$ \\
\hline
\end{tabular}

${ }^{a}$ Even for a highly soluble salt such as $\mathrm{KBiF}_{6}$ in aHF, the removal of $\mathrm{KF}$ contaminant could be achieved by removing most of the aHF under dynamic vacuum (the bulk of the $\mathrm{KBiF}_{6}$ crystallizing), the last few drops of aHF containing the bulk of any KF impurity then being removed by decantation. 
Table 3.3. X-ray powder data for $\mathrm{KRuF}_{6}$ with a rhombohedral unit cell.

\begin{tabular}{|c|c|c|c|c|c|c|c|c|}
\hline \multirow[b]{2}{*}{$\mathrm{I} / \mathrm{I}_{\mathrm{o}}$} & \multicolumn{2}{|c|}{$1 / d^{2} \times 10^{4}$} & \multicolumn{3}{|c|}{ rhombohedral } & \multicolumn{3}{|c|}{ hexagonal } \\
\hline & obsd. & calc. & $\mathrm{h}$ & $\mathrm{k}$ & 1 & $\mathrm{~h}$ & $\mathrm{k}$ & 1 \\
\hline $\mathrm{s}$ & 421 & 421 & 1 & 0 & 0 & 1 & 0 & 1 \\
\hline$s$ & 721 & 717 & 1 & 0 & -1 & 1 & 1 & 0 \\
\hline $\mathrm{s}$ & 974 & 967 & 1 & 1 & 0 & 0 & 1 & 2 \\
\hline $\mathrm{mw}$ & 1142 & 1138 & 1 & 1 & -1 & 0 & 2 & 1 \\
\hline $\mathrm{mw}$ & 1695 & 1684 & 2 & 0 & 0 & 2 & 0 & 2 \\
\hline $\mathbf{w}$ & 2161 & 2152 & 2 & -1 & -1 & 3 & 0 & 0 \\
\hline $\mathrm{s}$ & 2413 & 2401 & 2 & 1 & -1 & 1 & 2 & 2 \\
\hline $\mathrm{m}$ & 2879 & 2869 & 2 & 0 & -2 & 2 & 2 & 0 \\
\hline vw & 3157 & 3150 & 2 & 1 & 1 & 1 & 0 & 4 \\
\hline $\mathrm{w}$ & 3295 & 3290 & 2 & 1 & -2 & 1 & 3 & 1 \\
\hline $\mathbf{w}$ & 3811 & 3789 & 3 & 0 & 0 & 3 & 0 & 3 \\
\hline w & 3870 & 3867 & 2 & 2 & 0 & 0 & 2 & 4 \\
\hline $\mathrm{ms}$ & 4598 & 4584 & 3 & 1 & 0 & 2 & 1 & 4 \\
\hline $\mathrm{w}$ & 4723 & 4724 & 3 & 0 & -2 & 3 & 2 & 1 \\
\hline $\mathrm{m}$ & 5024 & 5020 & 2 & 1 & -3 & 1 & 4 & 0 \\
\hline $\mathrm{m}$ & 5280 & 5270 & 3 & 1 & -2 & 2 & 3 & 2 \\
\hline vvw & 5525 & 5504 & 3 & 1 & 1 & 2 & 0 & 5 \\
\hline vw & 6026 & 6019 & 3 & 2 & -1 & 1 & 3 & 4 \\
\hline vvw & 6473 & 6455 & 3 & 0 & -3 & 3 & 3 & 0 \\
\hline vwb & 6696 & 6704 & 4 & -1 & -1 & 5 & 0 & 2 \\
\hline vwb & 7448 & 7421 & 4 & 0 & -2 & 4 & 2 & 2 \\
\hline w & 11592 & 11570 & 5 & 1 & 0 & 4 & 1 & 6 \\
\hline vw & 11583 & 11570 & 5 & 1 & 0 & 4 & 1 & 6 \\
\hline w & 12451 & 12442 & 5 & 0 & -3 & 5 & 3 & 2 \\
\hline vw & 12446 & 12442 & 5 & 0 & -3 & 5 & 3 & 2 \\
\hline vw & 13020 & 13004 & 5 & 2 & -1 & 3 & 3 & 6 \\
\hline $\mathrm{w}$ & 14633 & 14625 & 5 & 2 & -3 & 3 & 5 & 4 \\
\hline vw & 14628 & 14625 & 5 & 2 & -3 & 3 & 5 & 4 \\
\hline vw & 14755 & 14751 & 5 & 2 & 1 & 3 & 1 & 8 \\
\hline vvw & 14751 & 14751 & 5 & 2 & 1 & 3 & 1 & 8 \\
\hline w & 15873 & 15873 & 5 & 3 & -2 & 2 & 5 & 6 \\
\hline $\mathrm{vw}$ & 15870 & 15873 & 5 & 3 & -2 & 2 & 5 & 6 \\
\hline $\mathbf{w}$ & 16188 & 16186 & 5 & 3 & 0 & 2 & 3 & 8 \\
\hline vwb & 16184 & 16186 & 5 & 3 & 0 & 2 & 3 & 8 \\
\hline
\end{tabular}


Table 3.4. X-ray powder data for $\mathrm{KRhF}_{6}$ with a rhombohedral unit cell.

\begin{tabular}{|c|c|c|c|c|c|c|c|c|}
\hline \multirow[b]{2}{*}{$\mathrm{I} / \mathrm{I}_{\mathrm{o}}$} & \multicolumn{2}{|c|}{$1 / d^{2} \times 10^{4}$} & \multicolumn{3}{|c|}{ rhombohedral } & \multicolumn{3}{|c|}{ hexagonal } \\
\hline & obsd. & calc. & $\mathrm{h}$ & $\mathrm{k}$ & 1 & $\mathrm{~h}$ & $\mathrm{k}$ & 1 \\
\hline $\mathrm{s}$ & 425 & 424 & 1 & 0 & 0 & 1 & 0 & 1 \\
\hline$s$ & 717 & 717 & 1 & 0 & -1 & 1 & 1 & 0 \\
\hline w & 865 & & & & & & & \\
\hline $\mathrm{s}$ & 982 & 981 & 1 & 1 & 0 & 0 & 1 & 2 \\
\hline $\mathrm{m}$ & 1138 & 1142 & 1 & 1 & -1 & 0 & 2 & 1 \\
\hline w & 1219 & & & & & & & \\
\hline $\mathrm{m}$ & 1698 & 1698 & 2 & 0 & 0 & 2 & 0 & 2 \\
\hline vw & 1862 & 1859 & 2 & 0 & -1 & 2 & 1 & 1 \\
\hline $\mathrm{w}$ & 2080 & & & & & & & \\
\hline w & 2150 & 2152 & 2 & -1 & -1 & 3 & 0 & 0 \\
\hline vs & 2419 & 2415 & 2 & 1 & -1 & 1 & 2 & 2 \\
\hline $\mathrm{mw}$ & 2875 & 2869 & 2 & 0 & -2 & 2 & 2 & 0 \\
\hline w & 3205 & 3205 & 2 & 1 & 1 & 1 & 0 & 4 \\
\hline $\mathrm{mw}$ & 3292 & 3293 & 2 & 1 & -2 & 1 & 3 & 1 \\
\hline vvw & 3457 & & & & & & & \\
\hline $\mathrm{mb}$ & 3838 & 3850 & 3 & 0 & -1 & 3 & 1 & 2 \\
\hline w & 3922 & 3922 & 2 & 2 & 0 & 0 & 2 & 4 \\
\hline$w$ & 4568 & 4567 & 2 & 2 & -2 & 0 & 4 & 2 \\
\hline $\mathrm{m}$ & 4641 & 4639 & 3 & 1 & 0 & 2 & 1 & 4 \\
\hline vw & 4731 & 4728 & 3 & 0 & -2 & 3 & 2 & 1 \\
\hline vvw & 4852 & & & & & & & \\
\hline $\mathrm{mw}$ & 5023 & 5021 & 3 & -1 & -2 & 4 & 1 & 0 \\
\hline $\mathrm{w}$ & 5282 & 5284 & 3 & 1 & -2 & 2 & 3 & 2 \\
\hline
\end{tabular}


Table 3.5. X-ray powder data for $\mathrm{LiIrF}_{6}$ with a rhombohedral unit cell.

\begin{tabular}{|c|c|c|c|c|c|c|c|c|c|c|c|}
\hline \multirow[b]{2}{*}{$\mathrm{I} / \mathrm{I}_{\mathrm{o}}$} & \multicolumn{2}{|c|}{$1 / d^{2} \times 10^{4}$} & \multirow[b]{2}{*}{$\mathrm{h}$} & \multirow[b]{2}{*}{$\mathrm{k}$} & \multirow[b]{2}{*}{1} & \multirow[b]{2}{*}{$\mathrm{I} / \mathrm{I}_{\mathrm{o}}$} & \multicolumn{2}{|c|}{$1 / \mathrm{d}^{2} \times 10^{4}$} & \multirow[b]{2}{*}{$\mathrm{h}$} & \multirow[b]{2}{*}{$\mathrm{k}$} & \multirow[b]{2}{*}{1} \\
\hline & obsd. & calc. & & & & & obsd. & calc. & & & \\
\hline $\mathrm{sb}$ & 486 & 485 & 1 & 1 & 1 & $w$ & 9325 & 9330 & 5 & 4 & 3 \\
\hline $\mathrm{vsb}$ & 576 & 575 & 1 & 0 & 0 & vw & 9418 & 9419 & 4 & 3 & 0 \\
\hline vsb & 740 & 737 & 1 & 1 & 0 & $\mathrm{vw}$ & 9951 & 9958 & 3 & 0 & -2 \\
\hline $\mathrm{sb}$ & 1388 & 1384 & 2 & 1 & 1 & vw & 10123 & 10120 & 3 & 1 & -2 \\
\hline $\mathrm{s}$ & 1547 & & & & & vw & 10169 & 10174 & 5 & 4 & 2 \\
\hline vw & 1576 & 1564 & 1 & 0 & -1 & vw & 10232 & 10228 & 5 & 2 & 1 \\
\hline $\mathrm{mw}$ & 1713 & & & & & $\mathrm{w}$ & 10631 & 10623 & 5 & 3 & 1 \\
\hline $\mathrm{m}$ & 1876 & 1869 & 2 & 2 & 1 & vvw & 10647 & 10623 & 5 & 3 & 1 \\
\hline $\mathrm{sb}$ & 2055 & 2049 & 2 & 1 & 0 & vw & 10773 & 10767 & 4 & 1 & -1 \\
\hline $\mathrm{mw}$ & 2150 & 2139 & 1 & 1 & -1 & $\mathrm{w}$ & 11275 & 11252 & 4 & 2 & -1 \\
\hline $\mathrm{mw}$ & 2307 & 2301 & 2 & 0 & 0 & vw & 11269 & 11252 & 4 & 2 & -1 \\
\hline vvw & 2399 & & & & & $\mathrm{w}$ & 11446 & 11432 & 3 & 2 & -2 \\
\hline $\mathrm{msb}$ & 2946 & 2948 & 2 & 2 & 0 & vw & 11449 & 11432 & 3 & 2 & -2 \\
\hline $\mathrm{mw}$ & 3174 & 3164 & 3 & 2 & 2 & vw & 11815 & 11792 & 4 & 4 & 0 \\
\hline $\mathbf{w}$ & 3444 & 3433 & 3 & 1 & 1 & $\mathrm{w}$ & 12187 & 12169 & 5 & 4 & 1 \\
\hline $\mathrm{ms}$ & 3516 & 3505 & 3 & 2 & 1 & $\mathrm{vw}$ & 12179 & 12169 & 5 & 4 & 1 \\
\hline $\mathrm{m}$ & 3722 & 3703 & 2 & 0 & -1 & $\mathrm{w}$ & 12468 & 12457 & 6 & 3 & 3 \\
\hline $\mathrm{m}$ & 3888 & 3865 & 2 & 1 & -1 & $\mathrm{w}$ & 12555 & 12547 & 5 & 2 & 0 \\
\hline$w$ & 3983 & 3973 & 3 & 3 & 2 & vw & 12778 & 12763 & 6 & 4 & 3 \\
\hline $\mathrm{vw}$ & 4331 & & 3 & 3 & 3 & $\mathrm{mw}$ & 12894 & 12888 & 4 & 3 & -1 \\
\hline vw & 4465 & & & & & $\mathrm{w}$ & 12899 & 12888 & 4 & 3 & -1 \\
\hline $\mathrm{mw}$ & 4538 & 4512 & 3 & 1 & 0 & $w$ & 13313 & 13302 & 6 & 3 & 2 \\
\hline mwb & 4721 & 4691 & 2 & -1 & -1 & $\mathrm{mw}$ & 13300 & 13302 & 6 & 3 & 2 \\
\hline $\mathrm{mw}$ & 5013 & 4997 & 3 & 2 & 0 & $\mathrm{mw}$ & 13366 & 13356 & 5 & 3 & 0 \\
\hline $\mathrm{mw}$ & 5186 & 5177 & 3 & 0 & 0 & vw & 13362 & 13356 & 5 & 3 & 0 \\
\hline $\mathrm{w}$ & 5557 & 5537 & 4 & 2 & 2 & vw & 13711 & 13698 & 6 & 5 & 4 \\
\hline $\mathrm{mb}$ & 5952 & 5932 & 4 & 3 & 2 & vw & 13906 & 13895 & 3 & 3 & -2 \\
\hline$w b$ & 6307 & 6291 & 4 & 2 & 1 & $\mathbf{w}$ & 14224 & 14219 & 6 & 5 & 3 \\
\hline vvw & 6512 & & & & & w & 14560 & 14560 & 4 & 1 & -2 \\
\hline vvw & 6644 & 6633 & 4 & 1 & 1 & vw & 14556 & 14560 & 4 & 1 & -2 \\
\hline vw & 6747 & 6741 & 3 & 1 & -1 & w & 14804 & 14811 & 4 & 0 & -2 \\
\hline vw & 6851 & 6830 & 2 & 1 & -2 & $\mathrm{w}$ & 14799 & 14811 & 4 & 0 & -2 \\
\hline vw & 7001 & 6992 & 3 & 0 & -1 & $\mathrm{mb}$ & 15303 & 15297 & 6 & 3 & 1 \\
\hline vw & 7110 & 7100 & 4 & 3 & 1 & $\mathrm{w}$ & 15311 & 15297 & 6 & 3 & 1 \\
\hline vvw & 7494 & 7478 & 4 & 4 & 2 & $\mathbf{w}$ & 15456 & 15458 & 4 & 2 & -2 \\
\hline vw & 7651 & 7639 & 3 & 2 & -1 & vw & 15459 & 15458 & 4 & 2 & -2 \\
\hline vw & 8125 & 8125 & 4 & 1 & 0 & $\mathrm{~m}$ & 15883 & 15890 & 1 & 3 & 13 \\
\hline $\mathrm{vw}$ & 8201 & 8197 & 4 & 2 & 0 & & & 15891 & 6 & 6 & 4 \\
\hline$w b$ & 9068 & 9060 & 5 & 2 & 2 & & & & & & \\
\hline
\end{tabular}




\subsection{References}

' P. R. Rao, R. Burbank, and N. Bartlett, (1968) unpublished data.

${ }^{2}$ N. K. Jha, Ph.D. Thesis, (1965) Chapter 2, U. British Columbia.

${ }^{3}$ N. Bartlett and D. H. Lohmann, J. Chem. Soc., February (1964), pp 619-626.

${ }^{4}$ J. L. Boston, and D. W. A. Sharp, J. Chem. Soc., (1960), p 907. 


\section{Chapter 4}

\section{The Oxidizing Chemistry of Solvated Ag ${ }^{\text {III }}$}

\subsection{Introduction}

Synthesis of the binary fluoride $\mathrm{AgF}_{3}$ was first claimed by Bougon and Lance ${ }^{1}$ in 1983. The red-brown material they described was generated by the oxidation of the metal or the mono- or difluoride of silver with $\mathrm{KrF}_{2}$ in $\mathrm{aHF}$ at room temperature. The material was reported to be weakly paramagnetic, but the data could be fit to the Curie law. In 1988, Kiselev et al ${ }^{2}$ claimed that the interaction of $\mathrm{AgF}_{2}$ with $\mathrm{O}_{2} \mathrm{~F}_{2}$ in $\mathrm{ClF}_{5}$ generated $\mathrm{AgF}_{3}$. These two materials, however, were not the same. Furthermore, neither was isomorphous with $\mathrm{AuF}_{3}$, which raised suspicion because of the structural similarity ${ }^{3}$ of $\mathrm{AgF}_{4}^{-}$and $\mathrm{AuF}_{4}^{-}$. Both of these claims were shown to be incorrect when, in 1989, a novel synthetic route to thermodynamically unstable binary fluorides such as $\mathrm{AgF}_{3}$ was found in these laboratories. ${ }^{4}$ The $\mathrm{AgF}_{3}$ prepared by this route was bright red and diamagnetic. Its XRPP closely resembled that of $\mathrm{AuF}_{3}$. Furthermore, a neutron diffraction study performed in 1991 demonstrated $^{5}$ a close structural relationship of $\mathrm{AgF}_{3}$ with that of 
$\mathrm{AuF}_{3}$. Other chemical and magnetic studies ${ }^{5}$ established that the material reported by Bougon and Lance as the trifluoride was $\mathrm{Ag}^{\mathrm{II}} \mathrm{Ag}_{2}^{\mathrm{III}} \mathrm{F}_{8}$ \{probably $\mathrm{Ag}^{2+}\left(\mathrm{AgF}_{4}^{-}\right)_{2}$ \}, and the similarity of the Kiselev et al. X-ray powder diffraction data to that of the Bougon and Lance material indicates that they also had prepared $\mathrm{Ag}_{3} \mathrm{~F}_{8}$ and not $\mathrm{AgF}_{3}$.

The successful synthetic route to the trifluoride of silver involved the abstraction of a fluoride ion from $\mathrm{AgF}_{4}^{-}$by the fluoroacid $\mathrm{BF}_{3}$ in $\mathrm{aHF}$ at or below room temperature. This synthesis was successful, in part, because it could be performed at low temperature, where the decomposition of the thermally unstable $\mathrm{AgF}_{3}$ was kinetically unfavorable. Genuine $\mathrm{AgF}_{3}$ loses ${ }^{5}$ elemental fluorine slowly in aHF to yield $\mathrm{Ag}_{3} \mathrm{~F}_{8}$ :

$$
3 \mathrm{AgF}_{3} \stackrel{\mathrm{aHF}}{\longrightarrow} \mathrm{Ag}_{3} \mathrm{~F}_{8}+1 / 2 \mathrm{~F}_{2}
$$

$\mathrm{AgF}_{3}$ was found ${ }^{5}$ to oxidize xenon gas spontaneously at room temperature according to the following equation:

$$
2 \mathrm{AgF}_{3}+\mathrm{Xe} \rightarrow 2 \mathrm{AgF}_{2}+\mathrm{XeF}_{2}
$$

Simple electrostatics predict that the electron affinity of neutral $\mathrm{AgF}_{3}$ is likely to be significantly less than that of a cationic $\mathrm{Ag}(\mathrm{III})$ species in aHF solution $\left\{\right.$ e.g. $\mathrm{AgF}_{2}(\mathrm{HF})_{\mathrm{n}}^{+}$, $\mathrm{AgF}(\mathrm{HF})_{\mathrm{n}}^{2+}$, or $\mathrm{Ag}(\mathrm{HF})_{\mathrm{n}}^{3+}$, where $\mathrm{HF}$ is assumed to coordinate to the $\mathrm{Ag}(\mathrm{III})$ via the electron-rich $\mathrm{F}$ ligand, $\mathrm{n}$ perhaps providing a total $\mathrm{Ag}(\mathrm{III})$ ligation of four\}. Such cations were assumed to be generated by the action of a strong $\mathrm{F}^{-}$acceptor. So an investigation was undertaken to determine the scope of the oxidizing capabilities of cationic $\mathrm{Ag}(\mathrm{III})$. No cationic $\operatorname{Ag}(\mathrm{III})$ fluoride is yet known to exist in the solid state. In fact, in 1989, 
attempts in this laboratory ${ }^{5}$ to synthesize $\mathrm{AgF}_{2}^{+} \mathrm{AsF}_{6}^{-}$at low temperature led to the formation of $\mathrm{AgFAsF}_{6}$ as illustrated in Equation (3),

$$
\mathrm{AgF}_{3} \underset{\mathrm{AsF}_{5}}{\stackrel{\mathrm{aHF}}{\longrightarrow}} \mathrm{AgFAsF}_{6}+1 / 2 \mathrm{~F}_{2}
$$

and eventually to the discovery of the remarkable oxidizing powers of both $\mathrm{Ag}(\mathrm{II})$ and $\mathrm{Ag}(\mathrm{III})$. Although the $\mathrm{AgF}_{2}^{+}$salt was never isolated, the production of $\mathrm{F}_{2}$ gas as a decomposition product suggested the ready liberation of elemental fluorine, which, in turn, suggested formidable oxidizing potential within the solution. Since the $F_{2}$ evolution

was slow, it was suspected that a transient $\mathrm{Ag}_{\text {solv }}^{\mathrm{III}}$ species could be generated in situ by the extraction of $\mathrm{F}^{-}$ions from $\mathrm{AgF}_{3}$ with the help of a strong fluoride ion acceptor. The work reported in this chapter provides clear evidence for long-lived cationic $\mathrm{Ag}(\mathrm{III})$ species in the aHF and their remarkable oxidizing capabilities. The strong acids $\mathrm{AsF}_{5}, \mathrm{SbF}_{5}$, and $\mathrm{BiF}_{5}$ in aHF produce the potent cationic $\mathrm{Ag}(\mathrm{III})$ oxidizers from $\mathrm{AgF}_{3}$, but the relatively weak acids $\mathrm{BF}_{3}$ and $\mathrm{PF}_{5}$ do not.

\subsection{Experimental}

\subsubsection{Preparation of $\mathrm{AMF}_{6}$}

See Chapter 3 for the details of alkali hexafluorometallate(V) syntheses. $\mathrm{XeF}_{5} \mathrm{AuF}_{6}$ was provided through a continuing collaboration with the Institute Jozef Stefan, Ljubljana, Slovenia. 


\subsubsection{Preparation of $\mathrm{KAgF}_{4}$}

$\mathrm{KAgF}_{4}$ was prepared according to the synthesis provided by Hoppe ${ }^{6} . \mathrm{AgNO}_{3}$ (3325 mg, $19.57 \mathrm{mmol})$ and $\mathrm{KNO}_{3}(1958 \mathrm{mg}, 19.36 \mathrm{mmol})$ were ground together in a mortar and pestle. The mixture was then placed into a nickel insert made to fit into a monel, high-pressure fluorine reactor. The insert was heated to $150^{\circ} \mathrm{C}$ for $15 \mathrm{~min}$ prior to being placed into the high-pressure reactor, which was sealed with a copper gasket and fitted with an Autoclave Engineers valve. After evacuating the reactor, it was cooled to $-196^{\circ} \mathrm{C}$ and $\sim 38 \mathrm{mmol} \mathrm{F}_{2}$ was admitted, thus bringing the internal pressure to $\sim 27 \mathrm{~atm}$ at room temperature. A resistance heater placed in a sand bath was used to heat the reactor to $\sim 300^{\circ} \mathrm{C}$ for $15 \mathrm{~h}$. After cooling down, all volatile species were removed from the reactor under dynamic vacuum. A liquid nitrogen bath was used to cool the reactor down once again to $-196^{\circ} \mathrm{C}$ and condense in $\sim 22 \mathrm{mmol} \mathrm{F}_{2}(16 \mathrm{~atm}$, in the reactor, at room temperature). This mixture was heated to $\sim 300^{\circ} \mathrm{C}$ for $15 \mathrm{~h}$, then cooled to room temperature. After removing the volatile products and unreacted $\mathrm{F}_{2}$, the reactor was brought into the DRILAB to be opened.

Scraping the solid product out of the nickel insert provided a mixture of orange and brown powder. This powder was placed into a passivated T-shaped FEP reactor. After evacuating the argon, aHF was condensed onto the mixture. At room temperature, the $\mathrm{KAgF}_{4}$ dissolved to give an orange solution, and a significant amount of brown solid remained undissolved. A single decantation was performed, after which all the aHF was 
removed under dynamic vacuum and the yellow-orange solid dried for $3 \mathrm{~h} .2000 \mathrm{mg}$ of solid were obtained, which gave the XRPP of $\mathrm{KAgF}_{4}\left(\right.$ tetragonal ${ }^{3}, \mathrm{a}_{\mathrm{o}}=5.90 \AA, \mathrm{c}_{\mathrm{o}}=11.15$ A) with some $\mathrm{KHF}_{2}$ as impurity.

Because of the synthesis used for the production of $\mathrm{AgF}_{3}$ (see below), in most cases it was not necessary to have $\mathrm{KAgF}_{4}$ which was free of $\mathrm{KHF}_{2}$. However, it was found that high purity $\mathrm{KAgF}_{4}$ could be obtained by first generating a room-temperature aHF solution of the $\mathrm{KAgF}_{4} / \mathrm{KHF}_{2}$ mixture, into which $\mathrm{PF}_{5}$ was slowly introduced with brisk agitation of the solution. At first sign of a persistent red precipitate, the $\mathrm{PF}_{5}$ supply was shut off. The weak fluoroacid complexes with the KF present much more readily than it displaces $\mathrm{AgF}_{3}$. And because $\mathrm{KPF}_{6}$ is only sparingly soluble in aHF, decantation provides a very clean sample, having sacrificed only a small quantity of $\mathrm{KAgF}_{4}$.

\subsubsection{Preparation of $\mathrm{AgF}_{3}$}

A T-shaped FEP reactor tube was loaded with $\mathrm{KAgF}_{4}$ (740 mg, $3.32 \mathrm{mmol}$ pure) and evacuated on the vacuum line. About $3 \mathrm{~mL}$ aHF was condensed onto the orange solid and warmed to room temperature. A very small quantity of brown precipitate was seen to settle out of solution, so the solution was decanted from it to the other arm of the reactor. The reactor was pressurized to $2 \mathrm{~atm}$ with $\mathrm{BF}_{3}$, leading to the immediate precipitation of red $\mathrm{AgF}_{3}$ from the aHF solution. More $\mathrm{BF}_{3}$ was introduced to replace that which was consumed in reaction. Once all the color had gone from the aHF solution, it was decanted. Over the next $3 \mathrm{~h}$, approximately twenty washings of the red product were performed by back-condensing the aHF extracting the $\mathrm{KBF}_{4}$ and decanting at 
approximately $0{ }^{\circ} \mathrm{C}$. The washed product was dried under dynamic vacuum for $3 \mathrm{~h}$. An XRPP of the red product shows only the lines of $\mathrm{AgF}_{3}$ \{hexagonal ${ }^{5}, \mathrm{a}_{\mathrm{o}}=5.0782(2) \AA, \mathrm{c}_{\mathrm{o}}$ $=15.4524(8) \AA\} ;$ yield $449 \mathrm{mg}(\sim 82 \%$ yield $)$.

\subsubsection{Oxidation of $\mathrm{RuF}_{6}^{-}$}

The following reagents were all loaded into one arm of a T-shaped FEP reactor: $\mathrm{LiRuF}_{6}(141 \mathrm{mg}, 0.633 \mathrm{mmol}), \mathrm{AgF}_{3}(219 \mathrm{mg}, 1.33 \mathrm{mmol}$ ), and BiF 5 (1187 mg, 3.903 mmol). Approximately $2.0 \mathrm{~mL}$ of anhydrous $\mathrm{HF}$ was condensed onto the solids at -196 ${ }^{\circ} \mathrm{C}$, then allowed to warm to $-25^{\circ} \mathrm{C}$. At this temperature, the solution was slightly yellow in color with a red solid resting at the bottom. No reaction was evident at this temperature. As the temperature was increased to room temperature, the HF quickly became deep red. For $12 \mathrm{~min}$, the reaction mixture was intermittently agitated as the deeply-colored, volatile material, $\mathrm{RuF}_{6}$, was evolved, coloring the liquid $\mathrm{HF}$ as well as the vapor in the reactor tubes. Then all volatile material was condensed into the other arm of the reactor at $-196^{\circ} \mathrm{C}$, leaving behind a deep green powder. An excess of dry oxygen gas was introduced into the reactor at this point, then the frozen volatile material was thawed. As the $\mathrm{aHF} / \mathrm{RuF}_{6}$ mixture melted, it first gave a red solution, which very quickly gave an almost colorless solution as the gaseous oxygen dissolved and precipitated dark red solid out of solution. Any remaining volatile compounds were pumped away and the red powder product was dried under dynamic vacuum for $3 \mathrm{~h}$ at 0 
${ }^{\circ} \mathrm{C}$. Powder X-ray diffraction confirmed the material to be $\mathrm{O}_{2} \mathrm{RuF}_{6}$ \{cubic ${ }^{7}, \mathrm{a}_{\mathrm{o}}=$ 10.004(3) $\AA$; ; the yield (91 mg) was $58 \%$.

\subsubsection{Oxidation of $\mathrm{RhF}_{6}^{-}$}

In the DRILAB, $\mathrm{KRhF}_{6}(87 \mathrm{mg}, 0.34 \mathrm{mmol}), \mathrm{KAgF}_{4}(140 \mathrm{mg}, 0.626 \mathrm{mmol})$, and $\mathrm{BiF}_{5}(828 \mathrm{mg}, 2.73 \mathrm{mmol}$ ) were mixed as solids in one tube of a passivated FEP Treactor. After removing the argon atmosphere under dynamic vacuum, about $2.2 \mathrm{~mL}$ aHF was condensed onto the solids and warmed to room temperature. Not until the reaction mixture had warmed almost completely to room temperature was there evidence of reaction. A small amount of bubbling occurred as the orange solution turned blood red. After $\sim 15 \mathrm{~min}$, all volatile species were condensed into the other arm of the reactor. Dry $\mathrm{O}_{2}$ was introduced and consumed as the red $\mathrm{RhF}_{6}$ warmed, forming $\mathrm{O}_{2} \mathrm{RhF}_{6}$. After removing the aHF under vacuum and drying the product for $2.5 \mathrm{~h}$, a red-brown $\mathrm{O}_{2} \mathrm{RhF}_{6}$ $(16 \mathrm{mg}, 0.062 \mathrm{mmol}$, yield $=18 \%)$ was recovered, as confirmed by X-ray powder photography $\left\{\right.$ cubic, $\mathrm{a}_{\mathrm{o}}=10.18(2) \AA$, see Table 4.1\}.

Table 4.1. X-ray powder data for $\mathrm{O}_{2} \mathrm{RhF}_{6}$ with a cubic unit cell.

\begin{tabular}{|c|c|c|c|}
\hline \multirow[b]{2}{*}{$\mathrm{I} / \mathrm{I}_{\mathrm{o}}$} & \multicolumn{2}{|c|}{$1 / \mathrm{d}^{2} \times 10^{4}$} & \multirow[b]{2}{*}{$\mathrm{h} \mathrm{k}$} \\
\hline & observed & calculated & \\
\hline $\mathrm{s}$ & 389 & 386 & 20 \\
\hline vs & 782 & 772 & 22 \\
\hline $\mathrm{vw}$ & 1150 & 1158 & 222 \\
\hline vvw & 1539 & 1544 & 40 \\
\hline $\mathrm{w}$ & 1943 & 1930 & 42 \\
\hline vvw & 2129 & 2123 & 332 \\
\hline $\mathrm{m}$ & 2335 & 2316 & 422 \\
\hline vw & 3057 & 3088 & 44 \\
\hline
\end{tabular}




\subsubsection{Oxidation of $\mathrm{PtF}_{6}^{-}$}

\subsubsection{Using $\mathrm{KPtF}_{6}$ as a Reagent}

In the DRILAB, $\mathrm{KPtF}_{6}(112 \mathrm{mg}, 0.321 \mathrm{mmol})$ and $\mathrm{KAgF}_{4}(161 \mathrm{mg}, 0.724 \mathrm{mmol})$ were loaded into the same tube of a passivated FEP T-reactor fitted with a Teflon valve. After the reactor was pumped down under dynamic vacuum, aHF $(1.5 \mathrm{~mL})$ was condensed onto the solid mixture. Upon warming to room temperature, the solution turned bright orange with some orange solid remaining undissolved. (Presumably, the highly-soluble $\mathrm{KAgF}_{4}$ completely dissolved to give the colored solution while some of the less soluble $\mathrm{KPtF}_{6}$ remained in the solid phase.) A small portion of aHF was condensed into the other arm of the reactor; no colored phase was condensed over with it, so it was poured back. The reactor was then pressurized to 1500 torr with $\mathrm{BF}_{3}$, which immediately precipitated a deep red solid from the solution. Additional $\mathrm{BF}_{3}$ was added until the reaction consumed no more and the reactor remained at 1500 torr. The resulting HF solution was light yellow in color, but there were no transferable colored volatile species. In order to increase the solubility of the $\mathrm{BF}_{3}$ in the $\mathrm{HF}$, the reactor was cooled down below $0{ }^{\circ} \mathrm{C}$ and agitated with no visible change. Again, performing the volatility test for colored material gave a negative result. So the majority of the $\mathrm{BF}_{3}$ was removed from the reactor by evacuating the reactor down to 750 torr (at which point the HF boiled). Then enough $\mathrm{AsF}_{5}$ was introduced into the reactor to bring its pressure up to 1500 torr. Formation of a blood-red solution in the aHF was immediately apparent. 
After allowing this reaction to continue for $10 \mathrm{~min}$, a volatility test yielded a deep red material in the side arm. So all volatile material was condensed into the side arm and frozen down to $-196^{\circ} \mathrm{C}$. The reactor was opened to a pressure of $\mathrm{O}_{2}$ gas and then closed again before being allowed to warm. As the deep-red mixture melted, it immediately reacted to give a yellow solution with some deep red precipitate. Upon removal of the $\mathrm{HF}$ and other volatile gases under dynamic vacuum at room temperature, the yield of dark red solid precipitate increased. This solid gave an XRPP, all lines of which matched that of $\mathrm{O}_{2} \mathrm{PtF}_{6}\left\{\right.$ cubic $^{8}, \mathrm{a}_{\mathrm{o}}=10.032(2) \AA$. The overall yield of $\mathrm{O}_{2} \mathrm{PtF}_{6}$ was $19.4 \%$.

\subsubsection{Using $\mathrm{LiPtF}_{6}$ as a Reagent}

The previously-described reaction was repeated using $\mathrm{LiPtF}_{6}$ instead of the less soluble $\mathrm{KPtF}_{6}$. Reagent quantities were as follows: $\mathrm{LiPtF}_{6}(100 \mathrm{mg}, 0.316 \mathrm{mmol}), \mathrm{AgF}_{3}$ (103 $\mathrm{mg}, 0.625 \mathrm{mmol}), \mathrm{BiF}_{5}(791 \mathrm{mg}, 2.60 \mathrm{mmol})$, aHF $(2.1 \mathrm{~mL})$. The reaction was agitated for about $12 \mathrm{~min}$ at room temperature. $\mathrm{O}_{2} \mathrm{PtF}_{6}(78 \mathrm{mg})$ was isolated in $72 \%$ yield, and the light-green byproduct (probably $\mathrm{Ag}\left(\mathrm{BiF}_{6}\right)_{2}$ and excess $\mathrm{BiF}_{5}$, with perhaps some $\mathrm{Ag}\left(\mathrm{BiF}_{6}\right)\left(\mathrm{PtF}_{6}\right)$, as well $\}$ remaining in the other arm of the reactor was measured to be $907 \mathrm{mg}$.

In a similar reaction, $\mathrm{AsF}_{5}$ was first used to generate $\mathrm{AgF}_{3}$ in situ, and then to activate the cationic silver(III) in order to affect the oxidation of $\mathrm{PtF}_{6}^{-}$. For this reaction, $\mathrm{LiPtF}_{6}(101 \mathrm{mg}, 0.320 \mathrm{mmol})$ and $\mathrm{KAgF}_{4}(135 \mathrm{mg}, 0.608 \mathrm{mmol})$ were combined, as solids, in one arm of a passivated FEP T-reactor. About $1.6 \mathrm{~mL}$ aHF was condensed onto 
the solids at $-196^{\circ} \mathrm{C}$, generating an orange solution above a small amount of undissolved solid at room temperature. $\mathrm{AsF}_{5}$ was slowly introduced into the reactor, first forming a red suspension. As more $\mathrm{AsF}_{5}$ was added, the red $\mathrm{PtF}_{6}$ gas was produced in copious amounts. After approximately 20 min reaction time, a liquid $\mathrm{N}_{2}$ bath was used to condense all volatile compounds into the other arm of the reactor. Then $\sim 1 \mathrm{mmol}_{2}$ gas was introduced into the reactor, leading to the formation of a red precipitate in an orange solution upon melting of the aHF. The aHF was removed as the reactor was evacuated, leaving the deep red $\mathrm{O}_{2} \mathrm{PtF}_{6}(82 \mathrm{mg}, 75 \%$ yield $)$ in one arm and a dark green-blue solid $\left(\mathrm{KAsF}_{6}, \mathrm{LiAsF}_{6}\right.$, and $\mathrm{AgFAsF}_{6}$, with perhaps some $\left.\mathrm{AgFPtF}_{6}\right)$ in the other arm.

\subsubsection{Attempted Oxidation of $\mathrm{AuF}_{6}^{-}$}

The following three reagents were all loaded into the same arm of a passivated FEP T-reactor: $\mathrm{XeF}_{5} \mathrm{AuF}_{6}(256.6 \mathrm{mg}, 0.4776 \mathrm{mmol}), \mathrm{AgF}_{3}(153.7 \mathrm{mg}, 0.9323 \mathrm{mg})$, and $\mathrm{BiF}_{5}(785.1 \mathrm{mg}, 2.583 \mathrm{mmol})$. Approximately $2.2 \mathrm{~mL}$ of aHF were condensed onto the mixed solids at $-196^{\circ} \mathrm{C}$ and then allowed to warm to $-40^{\circ} \mathrm{C}$. The solution appeared faint yellow with a red solid at the bottom. Intermittent agitation was maintained for $25 \mathrm{~min}$, during which time no sign of reaction was visible other than the occasional release of a bubble of colorless gas. Liquid nitrogen was used to test for the presence of a volatile, colored compound by condensing a very small amount of volatile material in the other arm of the reactor. This test came up negative, so the $-40^{\circ} \mathrm{C}$ bath was removed. As the reaction mixture warmed, it began to bubble vigorously, and the yellow solution began to 
turn green. Another test for volatile, colored material came up negative. The reaction was left to go to completion at room temperature over the next $1.5 \mathrm{~h}$. Then the HF was pumped away and the green solid product dried for 3 hours.

\subsection{Results and Discussion}

Experimental evidence which established that the electron affinity of $\mathrm{Ag}_{\text {solv }}^{\text {II }}$ is just a few kcal·mole ${ }^{-1}$ below that estimated for $\mathrm{PtF}_{6}$ (see Chapter 7) suggests that the much more potent trivalent species $\mathrm{Ag}_{\text {solv }}^{\mathrm{III}}$ has more than the requisite electron affinity to affect the oxidation of $\mathrm{PtF}_{6}^{-}$. Indeed, the rapid and vigorous evolution of blood-red $\mathrm{PtF}_{6}$ from an acidified solution of $\mathrm{Ag}_{\text {solv }}^{\mathrm{III}}$ and $\mathrm{PtF}_{6}^{-}$at ordinary temperatures confirms this prediction. $\mathrm{A}$ similar reaction was performed to demonstrate that $\mathrm{RuF}_{6}$, whose electron affinity is estimated to be approximately equal to that of $\mathrm{PtF}_{6}$, could also be liberated from its anion. The yield of $\mathrm{PtF}_{6}$ (measured gravimetrically after combination with $\mathrm{O}_{2}$ to form $\mathrm{O}_{2} \mathrm{PtF}_{6}$ ) was $75 \%$, while that of $\mathrm{RuF}_{6}$ was nearly $60 \%$. For the platinum and ruthenium reactions, the conditions were fairly well matched; however, a greater yield of $\mathrm{O}_{2} \mathrm{PtF}_{6}$ was measured. This observation is in accord with published results indicating that $\mathrm{O}_{2} \mathrm{PtF}_{6}$ and $\mathrm{PtF}_{6}$ are more stable than their ruthenium counterparts. Edwards et al. ${ }^{7}$ found that, upon heating a sample of $\mathrm{O}_{2} \mathrm{RuF}_{6}$, decomposition began at approximately $60^{\circ} \mathrm{C}$; and yet $\mathrm{O}_{2} \mathrm{PtF}_{6}$ remained intact to at least $100^{\circ} \mathrm{C}$. Furthermore, ruthenium pentafluoride was observed as the thermal decomposition product of $\mathrm{O}_{2} \mathrm{RuF}_{6}$, indicating ready loss of fluorine from the $\mathrm{RuF}_{6}$ molecule. $\mathrm{O}_{2} \mathrm{PtF}_{6}$ merely falls apart into molecular oxygen and 
the hexafluoride of platinum, the two of which spontaneously recombine at lower temperatures. Therefore, it is possible that some of the $\mathrm{RuF}_{6}$ which was generated in the aHF decomposed to $\mathrm{RuF}_{5}$ and $\mathrm{F}_{2}$ in solution ${ }^{9}$ as $\mathrm{O}_{2} \mathrm{RuF}_{6}$. The overall reaction scheme for the oxidation of $\mathrm{MF}_{6}^{-}$to $\mathrm{MF}_{6}$ is as follows:

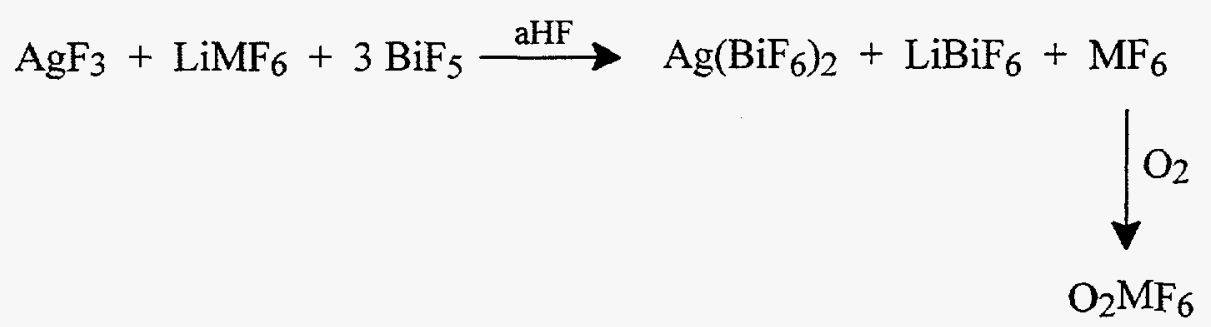

So the byproduct arm of the reactor should contain a mixture of $\mathrm{Ag}\left(\mathrm{BiF}_{6}\right)_{2}, \mathrm{LiBiF}_{6}$, and excess $\mathrm{BiF}_{5}$. Small quantities of metal pentafluoride, which are commonly poorly crystalline, should not be detected by XRPP in such a mixture of salts, and were not. Observations in this laboratory have determined that lithium hexafluorometallate(V) salts are generally significantly more soluble than their potassium counterparts in aHF solution. In addition, $\mathrm{LiRuF}_{6}$ has been qualitatively observed to be more soluble than $\mathrm{KPtF}_{6}$. These solubility considerations may account for the relatively low yield obtained in the oxidation of $\mathrm{KPtF}_{6}(-20 \%)$ as compared to those obtained in the oxidations of $\mathrm{LiPtF}_{6}(75 \%)$ and $\mathrm{LiRuF}_{6}(58 \%)$. It follows, then, that an extended reaction time could have increased the extent of oxidation of the $\mathrm{KPtF}_{6}$. However, the hexafluoride of platinum (as well of those of ruthenium, rhodium, and iridium) is seen to attack even well-passivated FEP and Teflon reactor parts within just a few minutes, so reaction time was kept to a minimum. 
The interaction of $\mathrm{AgF}_{3}$ and $\mathrm{XeF}_{5} \mathrm{AuF}_{6}$ in acidified aHF did not generate a colored, volatile species. At lower temperatures, in fact, there was no sign of reaction at all. This is unfortunate, since it is considered that the electron affinity of the Au center of the as yet unknown molecule $\mathrm{AuF}_{6}$ is sufficiently high that facile loss of a fluorine atom is likely to occur, even with moderate vibrational energy. All efforts to prepare $\mathrm{AuF}_{6}$ in hot reactions akin to those used for $\mathrm{MF}_{6}(\mathrm{M}=\mathrm{Pt}, \mathrm{Ru}, \mathrm{Rh})$ have failed. A lowtemperature electron oxidation of $\mathrm{AuF}_{6}^{-}$promised to be the best synthetic approach to $\mathrm{AuF}_{6}$. As the reaction mixture was warmed toward room temperature, all indications are that the $\mathrm{AgF}_{3}$ decomposed in the acid solution by the reaction expressed in Equation (4). The $\mathrm{XeF}_{5} \mathrm{AuF}_{6}$ merely provided $\mathrm{XeF}_{5}^{+}$and $\mathrm{AuF}_{6}^{-}$spectator ions to the system,

$$
\mathrm{AgF}_{3} \underset{\mathrm{BiF}_{5}}{\stackrel{\mathrm{aHF}}{\longrightarrow}} \mathrm{Ag}\left(\mathrm{BiF}_{6}\right)_{2}+1 / 2 \mathrm{~F}_{2}
$$

the oxidation of $\mathrm{AuF}_{6}^{-}$being out of reach of the cationic $\mathrm{Ag}_{\text {solv }}^{\mathrm{III}}$, the most powerfullyoxidizing silver system known. The green product recovered was probably a solid mixture of yellow $\mathrm{XeF}_{5} \mathrm{AuF}_{6}$ and blue-green $\mathrm{Ag}\left(\mathrm{BiF}_{6}\right)_{2}$, although XRPP showed a very faint pattern containing only those lines which belong to the silver salt.

It is pertinent that $\mathrm{KrF}^{+}$oxidizes ${ }^{10} \mathrm{O}_{2}$ to $\mathrm{O}_{2}^{+}$, and thus has an oxidizing potential comparable with $\mathrm{MF}_{6}(\mathrm{M}=\mathrm{Pt}, \mathrm{Ru}, \mathrm{Rh})$. However, because $\mathrm{KrFPtF}_{6}$ has been reported by Gillespie and Schrobilgen ${ }^{10}$ to decompose in solution with formation of $\mathrm{PtF}_{5}$, it appears that in aHF solution $\mathrm{KrF}^{+}$does not capture the electron from $\mathrm{PtF}_{6}^{-}$, but rather the $\mathrm{F}^{-}$ion. This result indicates that in aHF solution the $\mathrm{KrF}^{+}$is unable to electron oxidize $\mathrm{PtF}_{6}^{-}$, yet 
cationic $\mathrm{Ag}_{\text {solv }}^{\text {III }}$ is able to do so. Evidently, cationic $\mathrm{Ag}_{\text {solv }}^{\text {III }}$ is a more powerful oxidizer than $\mathrm{KrF}^{+}$in that same solvent. Indeed, the cationic $\mathrm{Ag}_{\text {solv }}^{\mathrm{III}}$, present in the aHF solutions containing strong $\mathrm{F}^{-}$acceptors such as $\mathrm{AsF}_{5}$ or $\mathrm{BiF}_{5}$, is the most potent chemical oxidizer so far discovered.

\subsection{Conclusions}

The red solid $\mathrm{AgF}_{3}$ is of very low solubility in aHF solution, although suspensions in aHF slowly lose $\mathrm{F}_{2}$. However, introduction of a strong fluoro-acid (e.g. $\mathrm{AsF}_{5}$ or $\mathrm{BiF}_{5}$ ) appears to capture fluoride from the $\mathrm{Ag}(\mathrm{III})$, permitting a cationic solution phase species, $\mathrm{Ag}_{\text {solv }}^{\text {III }}$, to be formed. With no appropriate reducing agent present, this cationic silver species eventually captures an electron from $\mathrm{F}^{-}$ion, or reductively eliminates fluorine, generating $\mathrm{F}_{2}$ gas and $\mathrm{Ag}(\mathrm{II})$. The lifetime of the cationic $\mathrm{Ag}_{\text {solv }}^{\mathrm{III}}$ is sufficiently long to provide for the production of $\mathrm{PtF}_{6}$ from $\mathrm{PtF}_{6}^{-}$in $75 \%$ yield, and the other platinum metal hexafluorides that are capable of oxidizing oxygen, $\mathrm{RuF}_{6}$ and $\mathrm{RhF}_{6}$, are also effectively liberated from their $\mathrm{MF}_{6}^{-}$anions. This cationic $\mathrm{Ag}_{\text {solv }}^{\mathrm{III}}$ species in $\mathrm{aHF}$ is a superior electron oxidizer to $\mathrm{KrF}^{+}$and therefore the most potent chemical oxidizer found so far. It does not liberate $\mathrm{AuF}_{6}$ from $\mathrm{AuF}_{6}^{-}$, however (see Chapter 1). 


\subsection{References}

${ }^{1}$ R. Bougon and M. Lance, C. R. Seances Acad. Sci., Ser. 2, 297 (1983), p 117.

${ }^{2}$ Y. M. Kiselev, A. I. Popov, A. A. Timakov, K. V. Bukharin, and V. F. Sukhoverkhov, Zh. Neorg. Khim., 33 (1988), p 1252.

${ }^{3}$ R. Hoppe and R. Homann, Z. Anorg. Allg. Chem., 379 (1970), p 193.

${ }^{4}$ B. Žemva, K. Lutar, A. Jesih, W. J. Casteel, Jr., and N. Bartlett, J. Chem. Soc., Chem. Comm., (1989), pp 346-347.

${ }^{5}$ B. Žemva, K. Lutar, A. Jesih, W. J. Casteel, Jr., A. P. Wilkinson, D. E. Cox, R. B. Von Dreele, H. Borrmann, and N. Bartlett, J. Am. Chem. Soc., 113 (1991), pp 4192-4198.

${ }^{6}$ R. Hoppe, Z. Anorg. Allg. Chem., 292 (1957), p 28.

${ }^{7}$ A. J. Edwards, W. E. Falconer, J. E. Griffiths, W. A. Sunder, and M. J. Vasile, J. Chem. Soc., Dalton Trans., (1974), pp 1129-1133.

${ }^{8}$ N. Bartlett and D. H. Lohmann, J. Chem. Soc., (1962), p 5253.

${ }^{9}$ In this laboratory, it has been observed that prolonged evacuation of $\mathrm{O}_{2} \mathrm{RuF}_{6}$ at room temperature generates a green solid which is very likely $\mathrm{RuF}_{5}$.

${ }^{10}$ R. J. Gillespie and G. J. Schrobilgen, J. Chem. Soc., Chem. Commun., (1974), p 90; and Inorg. Chem., 15 (1976), p 22. 


\section{Chapter 5}

\section{Synthesis and Oxidizing Properties of $\mathrm{AgF}^{+}$Salts}

\subsection{Introduction}

In 1982, Frlec et al. ${ }^{\mathrm{I}}$ reported the synthesis of $\mathrm{AgF}^{+} \mathrm{AsF}_{6}^{-}$, the first compound containing the silver(II) cation $\mathrm{AgF}^{+}$. Several years later, experimentation in this lab ${ }^{2}$ revealed that the interaction of $\mathrm{AgF}_{3}$ and $\mathrm{AsF}_{5}$ in aHF released elemental fluorine, leading to the formation of $\mathrm{AgFAsF}_{6}$ by way of the following reaction:

$$
\mathrm{AgF}_{3}+\mathrm{AsF}_{5} \rightarrow \mathrm{AgFAsF}_{6}+1 / 2 \mathrm{~F}_{2}
$$

An attempt to utilize the electron-withdrawing ability of the $\mathrm{Ag}(\mathrm{III})$ in this reaction to oxidize $\mathrm{Xe}$, however, did not result in the formation of the $\mathrm{Ag}(\mathrm{II})$ salt, but rather the $\mathrm{Ag}(\mathrm{I})$ salt, $\mathrm{AgAsF}_{6}$, and divalent Xe. Starting with $\mathrm{AgF}_{2}$ instead of $\mathrm{AgF}_{3}$, the following interaction was observed ${ }^{3}$ to occur:

$$
4 \mathrm{AgF}_{2}+5 \mathrm{AsF}_{5}+2 \mathrm{Xe} \rightarrow 4 \mathrm{AgAsF}_{6}+\mathrm{Xe}_{2} \mathrm{~F}_{3} \mathrm{AsF}_{6}
$$

Thus, it was concluded that, under acidic conditions in aHF at room temperature, $\mathrm{Ag}(\mathrm{II})$ was able to oxidize $\mathrm{Xe}(0)$ to $\mathrm{Xe}(\mathrm{II})$. A systematic search for other $\mathrm{AgF}^{+}$salts and an 
investigation into the oxidizing properties of this cation were undertaken and soon led to the discovery ${ }^{3}$ of the tetrafluoroborate analogue, and then the discovery ${ }^{4}$ of the tetrafluoroaurate(III) and the hexafluoroaurate(V) analogues.

In his studies, Casteel ${ }^{5}$ showed that the $(\mathrm{AgF})_{n}^{\mathrm{n}+}$ salts were less oxidizing than the blue solutions in aHF obtained by supplying additional acid to them. Since $(\mathrm{AgF})_{n}^{\mathrm{n}+}$ involves the addition of the electron-rich $\mathrm{F}^{-}$to $\mathrm{Ag}^{2+}$, the lower oxidizing potential of the complex cation relative to the $\mathrm{Ag}^{2+}$ is easily understood. It is less obvious that the solvated $\mathrm{Ag}^{2+}$ cation $\left(\mathrm{Ag}_{\text {solv }}^{\mathrm{II}}\right.$ ) would also be more potent than $(\mathrm{AgF})_{\mathrm{n}}^{\mathrm{n}+}$, but experimental evidence shows it to be so. ${ }^{6}$ However, the oxidizing power of the $(\mathrm{AgF})_{n}^{\mathrm{n}+}$ itself remained to be better defined.

In this chapter, the general question of which $\mathrm{AgF}^{+}$salts can be prepared is explored. A large part of this study was directed to $\mathrm{MF}_{6}^{-}$salts of third transition series elements because they provided a well-defined, nearly isodimensional set of anions, the oxidizability of which changes regularly across the series, and therefore responded to the question of the oxidizing power of $(\mathrm{AgF})_{\mathrm{n}}^{\mathrm{n}+}$. The synthetic work was accompanied always by X-ray powder diffraction studies supplemented wherever possible by single crystal investigations to discover the structures, or at least prove the purity, of the phases prepared. Magnetic measurements were also important in demonstrating that possible impurities such as $\mathrm{Ag}\left(\mathrm{MF}_{6}\right)_{2}$ salts (simple paramagnets ${ }^{7}$ ) or $\mathrm{AgF}_{2}$ (a ferromagnet ${ }^{9}, \mathrm{~T}_{\mathrm{c}}=$ 
$163 \mathrm{~K}$ ) were absent from the preparations, the magnetic susceptibility of $\mathrm{AgF}^{+}$salts characteristically exhibiting temperature-independence (see Experimental and Chapter 6).

\subsection{Experimental}

\subsubsection{Interaction of $\mathrm{LiOsF}_{6}$ with $\mathrm{F}_{2}$ in aHF}

A passivated FEP T-reactor was loaded with $\mathrm{LiOsF}_{6}(26 \mathrm{mg}, 0.084 \mathrm{mmol})$ and placed on the vacuum line. Approximately $1.2 \mathrm{~mL}$ aHF was condensed onto the colorless solid at $-196^{\circ} \mathrm{C}$, dissolving it completely upon warming to room temperature to give a colorless solution. The reactor was pressurized to $2 \mathrm{~atm}$ with $\mathrm{F}_{2}$ gas, following which the solution was cooled to near freezing (in order to increase the solubility of the gas in the $\mathrm{HF}$ ), then rewarmed with agitation. A liquid $\mathrm{N}_{2}$ bath was used to condense a small quantity of the $\mathrm{HF}$ into the other arm of the reactor. Because $\mathrm{OsF}_{6}$ is a volatile gas which appears bright yellow when condensed, the presence of any in the reactor would be made obvious by this test. In fact, no color was seen in the condensate. So the reaction mixture was agitated for $30 \mathrm{~min}$ and the test repeated. Again, a negative result indicated the absence of $\mathrm{OsF}_{6}$. The reactor was warmed to room temperature and all volatile compounds removed under vacuum; the $\mathrm{LiOsF}_{6}$ was recovered.

\subsubsection{Preparation of $\mathrm{AgF}_{2}$}

A passivated FEP T-reactor was loaded with AgF (566 mg, $4.46 \mathrm{mmol}$ ) which had been recrystallized from aHF. $1.5 \mathrm{~mL}$ aHF was condensed onto the orange solid, dissolving it at room temperature to give a slightly orange-tinted solution with a small 
quantity of brown solid left undissolved. This solution was decanted before $F_{2}$ was used to pressurize the reactor to $2 \mathrm{~atm}$ and continuous agitation was begun. Over the next few hours, more $\mathrm{F}_{2}$ was intermittently added as it was consumed in the reaction to give the brown, insoluble $\mathrm{AgF}_{2}$. After $6 \mathrm{~h}$ reaction time, the aHF was decanted and removed under vacuum. The brown powder was dried for more than $5 \mathrm{~h}$. An X-ray powder photograph of material prepared in this manner exhibits the XRPP of $\mathrm{AgF}_{2}$ $\left\{\right.$ orthorhombic $\left.{ }^{8}, a_{0}=5.568(1), b_{0}=5.831(1), c_{0}=5.101(1) \AA\right\}$ in a highly crystalline form ( $599 \mathrm{mg}, 92 \%$ yield). $\mathrm{AgF}_{2}$ displays a ferromagnetic susceptibility ${ }^{9}$, with a transition temperature of $163 \mathrm{~K}$ (see Figure 5.1 and Discussion below). $\mathrm{CaF}_{2}$ is used as a diamagnetic diluent in this sample because of the very large magnitude of the volume susceptibility of $\mathrm{AgF}_{2}$ below its transition temperature. 


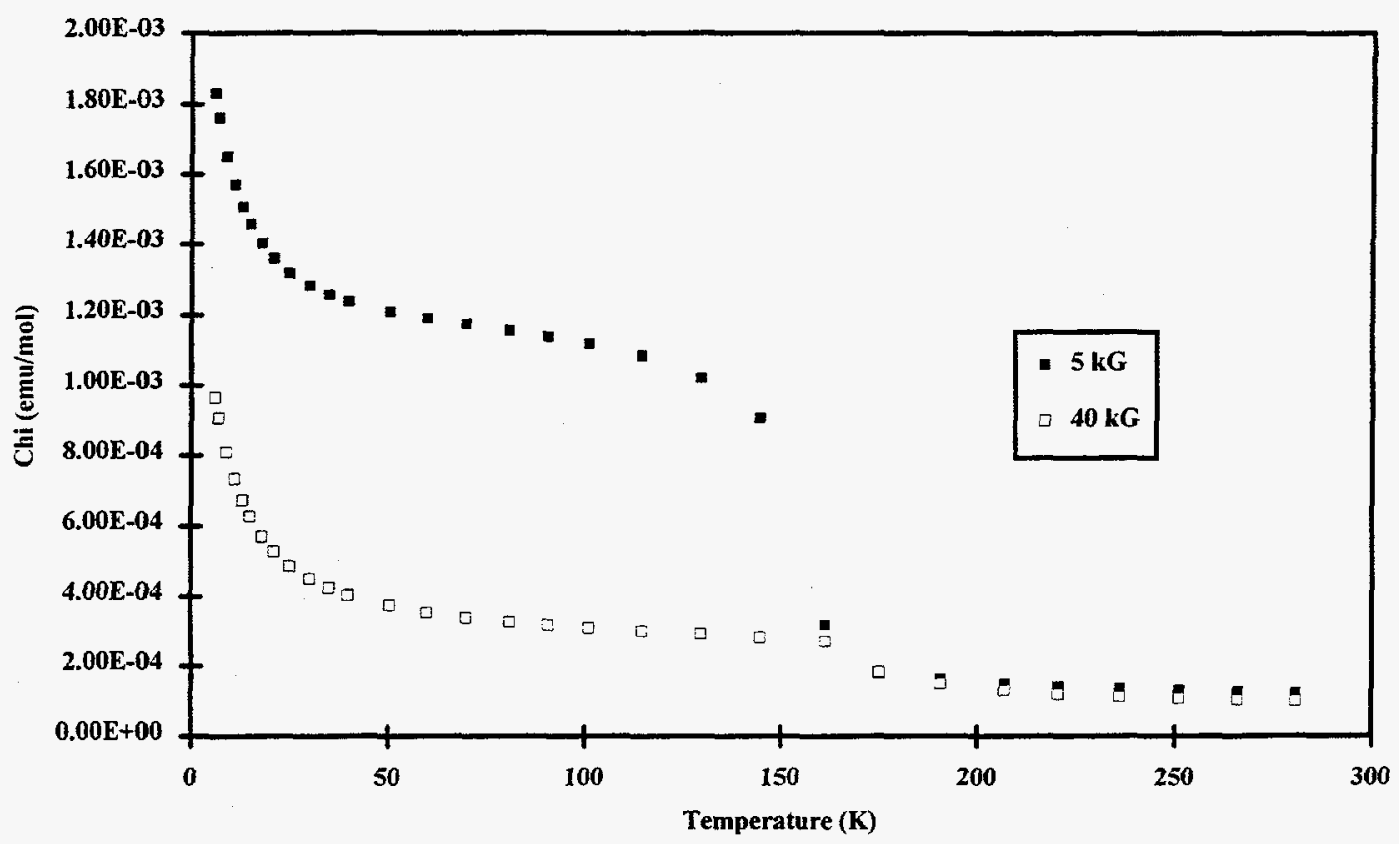

Figure 5.1. Magnetic data for mixture of $15 \% \mathrm{AgF}_{2}, 85 \% \mathrm{CaF}_{2}$.

\subsubsection{Preparation of $\operatorname{Ag}\left(\mathrm{BiF}_{6}\right)_{2}$}

Recrystallized $\mathrm{AgF}(141 \mathrm{mg}, 1.11 \mathrm{mmol})$ and $\mathrm{BiF}_{5}(748 \mathrm{mg}, 2.46 \mathrm{mmol})$ were loaded into separate tubes of a passivated FEP T-reactor inside the DRILAB. After evacuating the argon gas from the reactor, approximately $3 \mathrm{~mL}$ aHF was condensed onto the $\mathrm{AgF}$, dissolving it completely after warming to room temperature. Fluorine gas was repeatedly introduced into the reactor as it was consumed in the production of $\mathrm{AgF}_{2}$ (see $\mathrm{AgF}_{2}$ synthesis above). After all signs of reaction had ceased, the reactor was evacuated to 1000 torr. Then the solid $\mathrm{BiF}_{5}$ was shaken over into the reaction tube. Turquoise solid was seen to form immediately on the walls of the reactor tube. A small quantity of aHF was condensed over into the other tube and used to rinse over any remaining $\mathrm{BiF}_{5}$. The 
reaction mixture was agitated continuously for $15 \mathrm{~h}$. The colorless aHF solution was then decanted from the turquoise solid and all volatile species were pumped away under dynamic vacuum. An X-ray powder photograph of the product $(835 \mathrm{mg}, 99.7 \%$ yield) gave the XRPP of $\mathrm{Ag}\left(\mathrm{BiF}_{6}\right)_{2}$, as shown in Table 5.1. A single crystal X-ray structure solution was later performed in this laboratory, permitting extensive indexing of the powder pattern observed. Magnetic susceptibility measurements performed on this material (see Figure 6.8) show Curie-Weiss behavior above $\sim 50 \mathrm{~K}$, with a magnetic moment of approximately 2.2 B.M. This value is in exact agreement with that calculated ${ }^{10}$ for $\mathrm{Cu}^{2+}$ assuming free-ion values of spin-orbit coupling.

Table 5.1. X-ray powder data for $\mathrm{Ag}\left(\mathrm{BiF}_{6}\right)_{2}$ with a triclinic unit cell. $a_{0}=5.217(2) \AA, b_{0}=5.583(3) \AA, c_{0}=8.948(5) \AA$, $\alpha=76.01(3)^{\circ}, \beta=88.98(3)^{\circ}, \gamma=65.12(3)^{\circ}, V=228.4 \AA^{3}$.

\begin{tabular}{|c|c|c|c|c|c|c|c|}
\hline \multicolumn{2}{|c|}{$\mathrm{I} / \mathrm{I}_{\mathrm{o}}$} & \multicolumn{3}{|c|}{$1 / \mathrm{d}^{2} \times 10^{4}$} & \multirow[b]{2}{*}{$\mathrm{h}$} & \multirow[b]{2}{*}{$\mathrm{k}$} & \multirow[b]{2}{*}{1} \\
\hline observed & calculated $^{a}$ & observed & corrected $^{b}$ & calculated $^{c}$ & & & \\
\hline $\mathrm{m}$ & 55 & 425 & 420 & 418 & 0 & 1 & 0 \\
\hline $\mathrm{m}$ & 19 & 456 & 452 & 451 & 1 & 0 & 0 \\
\hline \multirow[t]{3}{*}{ vs } & 36 & 542 & 537 & 535 & 0 & 0 & 2 \\
\hline & 40 & & & 538 & 1 & 0 & -1 \\
\hline & 37 & & & 554 & 1 & 1 & 1 \\
\hline vs & 100 & 639 & 633 & 631 & 1 & 0 & 1 \\
\hline \multirow[t]{2}{*}{ vs } & 90 & 718 & 712 & 705 & 1 & 1 & -1 \\
\hline & 87 & & & 709 & 0 & 1 & 2 \\
\hline \multirow[t]{3}{*}{ vs } & 39 & 1216 & 1209 & 1197 & 0 & 1 & -2 \\
\hline & 11 & & & 1205 & 0 & 0 & 3 \\
\hline & 41 & & & 1205 & 1 & -1 & -1 \\
\hline $\mathrm{m}$ & 36 & 1321 & 1313 & 1313 & 1 & 2 & 1 \\
\hline \multirow[t]{2}{*}{$\mathrm{m}$} & 16 & 1479 & 1471 & 1475 & 2 & 1 & 0 \\
\hline & 20 & & & 1475 & 1 & 1 & 3 \\
\hline w & 17 & 1526 & 1518 & 1515 & 1 & 0 & -3 \\
\hline vvw & 7 & 1727 & 1718 & 1718 & 0 & 2 & 2 \\
\hline w & 17 & 1813 & 1804 & 1802 & 2 & 0 & 0 \\
\hline
\end{tabular}




\begin{tabular}{|c|c|c|c|c|c|c|c|}
\hline \multicolumn{2}{|c|}{$I / I_{0}$} & \multicolumn{3}{|c|}{$1 / d^{2} \times 10^{4}$} & \multirow[b]{2}{*}{$\mathrm{h}$} & \multirow[b]{2}{*}{$\mathrm{k}$} & \multirow[b]{2}{*}{1} \\
\hline observed & calculated $^{\mathrm{a}}$ & observed & corrected $^{b}$ & calculated $^{c}$ & & & \\
\hline$\overline{\mathrm{Vw}}$ & 16 & 1927 & 1919 & 1926 & 1 & 1 & -3 \\
\hline vw & 18 & 1995 & 1986 & 1989 & 1 & 2 & 3 \\
\hline vvw & 5 & 2072 & 2062 & 2067 & 2 & 1 & -2 \\
\hline $\mathrm{m}$ & 17 & 2161 & 2152 & 2150 & 2 & 0 & -2 \\
\hline $\mathbf{w}$ & 15 & 2224 & 2215 & 2217 & 2 & 2 & 2 \\
\hline vvw & & 2410 & 2400 & & & & \\
\hline $\mathrm{m}$ & 12 & 2534 & 2524 & 2525 & 2 & 0 & 2 \\
\hline vw & 6 & 2606 & 2596 & 2594 & 2 & 1 & 3 \\
\hline$v w$ & 5 & 2730 & 2720 & 2729 & 1 & 2 & 4 \\
\hline $\mathbf{w}$ & 13 & 2839 & 2829 & 2836 & 0 & 2 & 4 \\
\hline $\mathrm{m}$ & 18 & 2977 & 2966 & 2965 & 2 & -1 & 0 \\
\hline vvw & 4 & 3066 & 3056 & 3069 & -2 & 1 & 2 \\
\hline $\mathrm{m}$ & 13 & 3208 & 3197 & 3198 & -1 & 2 & 3 \\
\hline \multirow[t]{2}{*}{$\mathbf{m}$} & 14 & 3347 & 3336 & 3327 & 2 & 3 & 0 \\
\hline & 13 & & & 3339 & 1 & 3 & 3 \\
\hline w & 8 & 3517 & 3506 & 3508 & 3 & 1 & 1 \\
\hline \multirow[t]{2}{*}{$\mathrm{w}$} & 2 & 3594 & 3583 & 3570 & 2 & 0 & -4 \\
\hline & 5 & & & 3608 & 0 & -2 & 3 \\
\hline \multirow[t]{2}{*}{$\mathrm{m}$} & 7 & 3763 & 3752 & 3736 & 1 & 2 & 5 \\
\hline & 6 & & & 3743 & -1 & 1 & 5 \\
\hline vVw & 2 & 3955 & 3944 & 3932 & 2 & -1 & 2 \\
\hline $\mathbf{w}$ & & 4070 & 4059 & & & & \\
\hline vw & 2 & 4288 & 4277 & 4259 & 0 & -3 & 1 \\
\hline $\mathrm{w}$ & 8 & 4401 & 4390 & 4384 & 3 & 2 & 3 \\
\hline w & 11 & 4530 & 4519 & 4505 & 3 & 1 & -3 \\
\hline vvw & 2 & 4692 & 4681 & 4681 & 2 & 0 & -5 \\
\hline $\mathrm{vw}$ & 4 & 4867 & 4855 & 4863 & 1 & 1 & 6 \\
\hline $\mathrm{vw}$ & 5 & 5054 & 5042 & 5048 & -1 & 3 & 1 \\
\hline vvw & 1 & 5173 & 5161 & 5154 & -2 & 2 & 3 \\
\hline rvw & 5 & 5254 & 5242 & 5251 & 2 & 4 & 2 \\
\hline vw & 5 & 5363 & 5351 & 5336 & 1 & 4 & 1 \\
\hline vvw & 2 & 5450 & 5439 & 5434 & 2 & -2 & 1 \\
\hline vvw & 4 & 5610 & 5598 & 5617 & 2 & 0 & 5 \\
\hline vvw & 3 & 5916 & 5904 & 5900 & 4 & 2 & 0 \\
\hline vvw & 2 & 6073 & 6062 & 6060 & 2 & 0 & -6 \\
\hline Vvw & 3 & 6209 & 6197 & 6172 & 1 & -2 & 4 \\
\hline vVw & 1 & 6384 & 6373 & 6380 & 0 & 3 & 6 \\
\hline \multirow[t]{2}{*}{ w } & 2 & 6561 & 6550 & 6559 & 2 & 3 & -4 \\
\hline & 2 & & & 6559 & 0 & 0 & 7 \\
\hline
\end{tabular}




\begin{tabular}{|c|c|c|c|c|c|c|c|}
\hline \multicolumn{2}{|c|}{$\mathrm{I} / \mathrm{I}_{\mathrm{o}}$} & \multicolumn{3}{|c|}{$1 / d^{2} \times 10^{4}$} & \multirow[b]{2}{*}{ h } & \multirow[b]{2}{*}{$\mathrm{k}$} & \multirow[b]{2}{*}{1} \\
\hline observed & calculated $^{\mathrm{a}}$ & observed & corrected $^{b}$ & calculated $^{\mathrm{c}}$ & & & \\
\hline \multirow[t]{2}{*}{$w$} & 5 & 6699 & 6687 & 6683 & 0 & 4 & 0 \\
\hline & 2 & & & 6689 & 1 & -3 & 2 \\
\hline vw & 1 & 6802 & 6791 & 6794 & 3 & 1 & 5 \\
\hline vvw & 2 & 6923 & 6912 & 6934 & 4 & 2 & 3 \\
\hline vvw & 2 & 7091 & 7079 & 7074 & 1 & -1 & 6 \\
\hline vw & 2 & 7212 & 7201 & 7209 & 4 & 0 & 0 \\
\hline $\mathbf{w}$ & 2 & 7404 & 7393 & 7389 & -1 & 2 & 7 \\
\hline vvw & 1 & 7555 & 7544 & 7537 & 4 & 1 & 3 \\
\hline vvw & 2 & 7836 & 7825 & 7814 & 4 & 2 & 4 \\
\hline vw & 2 & 8292 & 8282 & 8270 & 4 & 2 & -4 \\
\hline vw & 2 & 8609 & 8598 & 8602 & 4 & 0 & -4 \\
\hline vvw & 2 & 8952 & 8941 & 8937 & 0 & -3 & 5 \\
\hline vvw & 2 & 9030 & 9020 & 9000 & 3 & 4 & 6 \\
\hline vvw & 1 & 9349 & 9339 & 9353 & 0 & -4 & 3 \\
\hline vvw & 2 & 9391 & 9381 & 9384 & -3 & 2 & 5 \\
\hline vvw & 2 & 9699 & 9689 & 9702 & 5 & 3 & -1 \\
\hline vvw & 1 & 9795 & 9786 & 9787 & 3 & 1 & -7 \\
\hline vvw & 1 & 10463 & 10454 & 10445 & 5 & 2 & -3 \\
\hline vvw & 3 & 10853 & 10844 & 10851 & -3 & 3 & 3 \\
\hline
\end{tabular}

${ }^{a}$ Calculated intensities are based on cell parameters and atom positions provided in the single crystal structure solution.

${ }^{b}$ An absorption correction has been applied to the line positions.

${ }^{c}$ Calculated line positions are based on cell parameters and atom positions provided by a refinement on the powder data presented here.

\subsubsection{Preparation of $\mathrm{AgBF}_{4}$}

A passivated FEP T-reactor was loaded with $\sim 1 \mathrm{gm} \mathrm{AgF}$ as received. After condensing $\sim 2 \mathrm{~mL}$ aHF onto the $\mathrm{AgF}$, the solution was warmed to room temperature. Probably due to the light sensitivity of AgF, there is always some brown solid which slowly settles out of solution, but everything else dissolves to give a slightly orangecolored solution. After allowing several hours for the brown solid to settle, the HF 
solution was decanted into the other arm of the reactor. $\mathrm{BF}_{3}$ was then introduced into the reactor, immediately forming colorless needles. As the $\mathrm{BF}_{3}$ was consumed, more was intermittently added until no more needles could be seen to form. Then the reactor was pressurized to 1500 torr with $\mathrm{BF}_{3}$ and agitated continuously for over $2 \mathrm{~h}$. The $\mathrm{HF} / \mathrm{BF}_{3}$ was then removed and the colorless product dried for approximately $3 \mathrm{~h}$. X-ray powder photography was used to confirm the identity and purity of the $\mathrm{AgBF}_{4}$ product (orthorhombic ${ }^{11}, a_{0}=8.157, b_{0}=5.345, c_{o}=6.792 \AA$ ).

\subsubsection{Preparation of $\mathrm{AgFBF}_{4}$}

\subsubsection{Violet Colored $\mathrm{AgFBF}_{4}$}

A passivated FEP T-reactor was loaded with $\mathrm{AgBF}_{4}$. Approximately 1 to $2 \mathrm{~mL}$ aHF was condensed onto the white solid, only partially dissolving it at room temperature. The reactor was pressurized to $\sim 900$ torr with $\mathrm{BF}_{3}$ and then to 1500 torr with $\mathrm{F}_{2}$ gas, and the solution was mechanically agitated or stirred. More $F_{2}$ gas was periodically introduced to replace that which was consumed in the reaction as the white solid slowly turned gray-blue. Mixing was continued for at least $12 \mathrm{~h}$, until no more $F_{2}$ uptake was observed. Most of the $F_{2}$ was then removed from the reactor by slowly pumping on the reactor until its internal pressure dropped to 800 torr at room temperature. At this point, the reactor was pressurized to 2 atm with $\mathrm{BF}_{3}$, which slightly darkened the blue tint of the solution. This mixture was left to sit for $15 \mathrm{~min}$. Then the product was dried as fully as possible by decantation. All volatile species present were pumped away and the grayblue product dried for over $3 \mathrm{~h}$ under dynamic vacuum. X-ray powder photography of 
material prepared in this manner gives the XRPP expected of pure $\mathrm{AgFBF}_{4}$ \{tetragonal ${ }^{4}$, $\left.a_{0}=6.7000(3), c_{0}=4.0113(2) \AA\right\}$. Magnetic susceptibility data for this material were obtained (Figure 5.2), showing it to be essentially free of $\mathrm{AgF}_{2}$, and to have a very small magnitude temperature-independent paramagnetism.

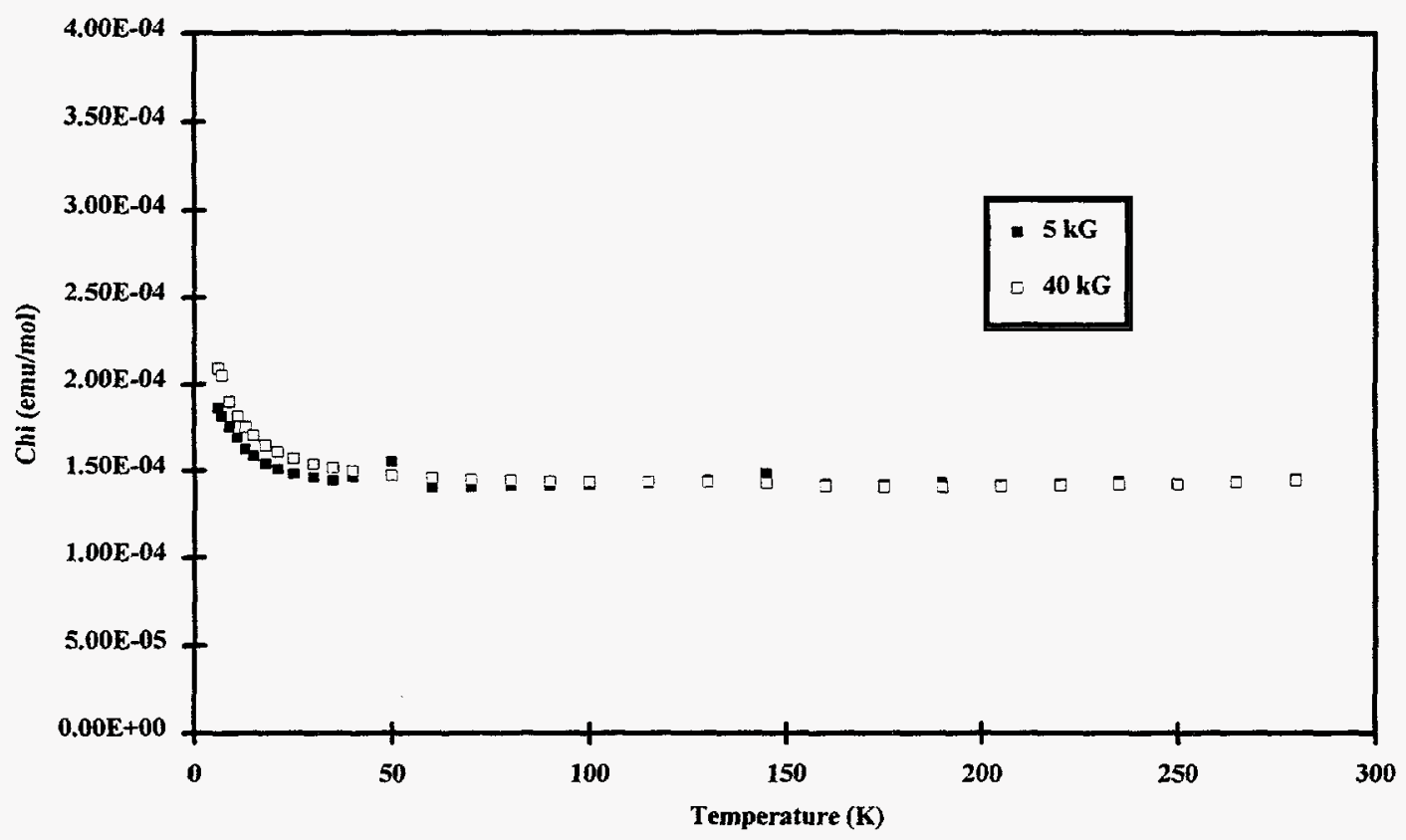

Figure 5.2. Magnetic data for $\mathrm{AgFBF}_{4}$.

\subsubsection{Bronze Colored AgFBF 4}

Several different proportions for the two solid reagents were tried, the most successful of which is described. A passivated FEP T-reactor was loaded with $\mathrm{AgBF}_{4}$ (149 $\mathrm{mg}, 0.765 \mathrm{mmol}$ ) and $\mathrm{AgF}$ ( $5 \mathrm{mg}, 0.04 \mathrm{mmol}$ ) in the same arm. After evacuating the reactor, $1 \mathrm{~mL}$ aHF was condensed onto the solids. The reactor was pressurized to 
1000 torr with $F_{2}$ and intermittently agitated. A bronze precipitate formed immediately and the $\mathrm{HF}$ turned very light blue. After just 15 min of intermittent agitation, the $\mathrm{HF} / \mathrm{F}_{2}$ was removed and the bronze crystalline material dried for $3 \mathrm{~h}$. An X-ray photograph reveals only the patterns of $\mathrm{AgFBF}_{4}$ and $\mathrm{AgF}_{2}$. Macroscopic crystals of this bronze material were visible, one of which was selected for single crystal X-ray structure determination (see Chapter 6).

Similar results were obtained when $\mathrm{AgF}_{2}$ was used in place of $\mathrm{AgF}$, although the crystallinity was noticeably worse.

\subsubsection{Preparation of $\mathrm{AgAsF}_{6}$}

A passivated FEP T-reactor was loaded with recrystallized AgF (385 mg, 3.04 mmol). $1.5 \mathrm{~mL}$ aHF was condensed onto the solid and warmed to room temperature. A very small quantity of undissolved, brown solid was visible in a slightly orange solution. After letting the brown solid settle, the solution was decanted into the other arm of the reactor. $\mathrm{AsF}_{5}$ was introduced into the reactor and immediately formed a colorless powder precipitate. Over the next $2 \mathrm{~h}$ of intermittent agitation, the $\mathrm{AsF}_{5}$ pressure was periodically restored to $\sim 1500$ torr as it was consumed in the reaction. The HF solution was decanted and the HF back-distilled onto the white solid. Two more such washings of the precipitate were performed prior to drying the $\mathrm{AgAsF}_{6}$ product $(861 \mathrm{mg}, 96 \%$ yield) under dynamic vacuum for 3 hours. Material prepared in this manner gives an XRPP indicative of pure $\mathrm{AgAsF}_{6}\left(\mathrm{cubic}^{12}, \mathrm{a}_{0}=7.74 \AA\right)$. 


\subsubsection{Preparation of AgFAsF $_{6}$}

A passivated FEP T-reactor fitted with $1 / 2$ " tubes was loaded with $\mathrm{AgAsF}_{6}$ (734 $\mathrm{mg}, 2.47 \mathrm{mmol}$ ). $3 \mathrm{~mL}$ aHF was condensed onto the white solid, dissolving very little of it. The reactor was pressurized to 1000 torr with $\mathrm{AsF}_{5}$ and then to 1500 torr with $\mathrm{F}_{2}$. Continuous mechanical agitation was begun, and as the reaction consumed $\mathrm{F}_{2}$, more was intermittently added. After $15 \mathrm{~h}$ of reaction time, $\mathrm{F}_{2}$ uptake had ceased; the solid was aqua blue and the solution a slightly deeper blue. After decanting the $\mathrm{HF}$, the volatile species were pumped out and the $\mathrm{AgFAsF}_{6}$ product ( $709 \mathrm{mg}, 91 \%$ yield) dried for over 3 h. X-ray powder photography of material prepared in this manner shows only the XRPP of AgFAsF 6 \{orthorhombic $\left.{ }^{13}, a_{o}=7.585(1), b_{o}=6.997(6), c_{o}=9.852(1) \AA\right\}$. The magnetic susceptibility of this material is shown in Figure 5.3, in which the signal is essentially temperature-independent above $\sim 100 \mathrm{~K}$. A paramagnetic impurity may be responsible for the low-temperature rise in susceptibility. 


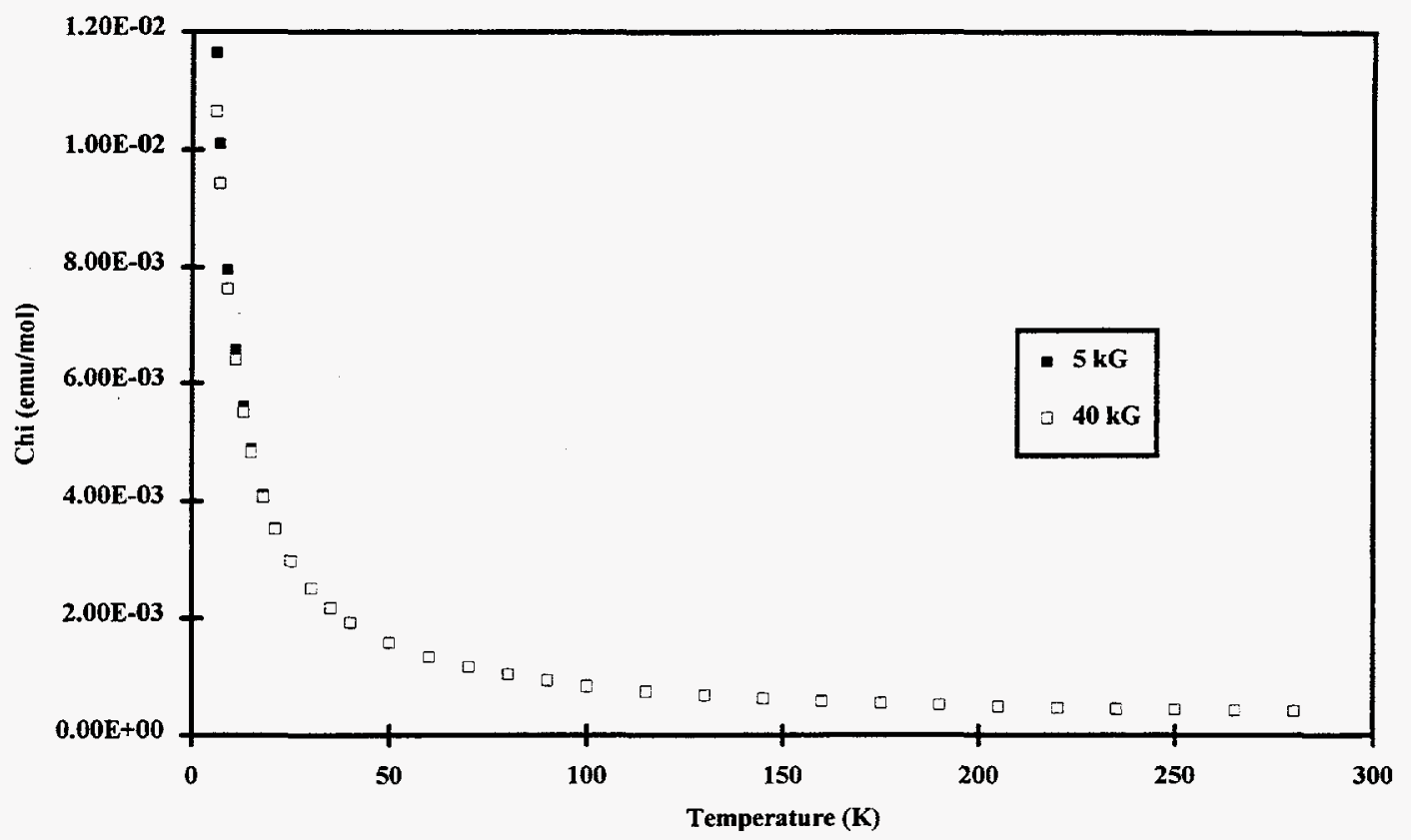

Figure 5.3. Magnetic data for $\mathrm{AgFAsF}_{6}$.

\subsubsection{Preparation of $\mathrm{AgBiF}_{6}$}

A passivated FEP T-reactor was loaded with $\mathrm{AgBF}_{4}(206 \mathrm{mg}, 1.06 \mathrm{mmol})$ in one tube and $\mathrm{BiF}_{5}(347 \mathrm{mg}, 1.14 \mathrm{mmol})$ in the other. Approximately $1.0 \mathrm{~mL}$ aHF was condensed onto each of the reagents, dissolving all of the $\mathrm{BiF}_{5}$, but only a small portion of the $\mathrm{AgBF}_{4}$. As the $\mathrm{BiF}_{5}$ solution was slowly poured into the $\mathrm{AgBF}_{4}$ solution, there was vigorous gas evolution and precipitation of a light yellow solid. The gas evolution quickly abated, so some of the $\mathrm{BF}_{3}$ pressure was released into the vacuum line before continuous agitation of the reaction mixture was begun. After $30 \mathrm{~min}$, the solution was cooled to $0{ }^{\circ} \mathrm{C}$ and decanted. The $\mathrm{HF}$ (containing some $\mathrm{BF}_{3}$ ) was then condensed back 
over at $-196^{\circ} \mathrm{C}$, warmed to $0^{\circ} \mathrm{C}$, and then decanted again. The volatile species were pumped away and the light yellow product dried for 3 hours. An X-ray powder photograph of the product shows an XRPP (see Table 5.2) related to those of $\mathrm{AgRuF}_{6}$, $\mathrm{AgOsF}_{6}, \mathrm{AgIrF}_{6}$, and $\mathrm{AgSbF}_{6}$, which $\mathrm{Kemmitt}_{\text {et }}$ al. ${ }^{14}$ report as the $\mathrm{KNbF}_{6}$ structure type. The indexing provided in Table 5.2 is based upon this tetragonal structure type and yields reasonable lattice cell parameters for the formulation $\mathrm{AgBiF}_{6}(467 \mathrm{mg}, 102 \%$ yield). 
Table 5.2. X-ray powder pattern of $\mathrm{AgBiF}_{6}$ with a tetragonal unit cell.

\begin{tabular}{|c|c|c|c|c|c|c|c|c|c|c|c|}
\hline \multirow[b]{2}{*}{$\mathrm{I} / \mathrm{I}_{\mathrm{o}}$} & \multicolumn{2}{|c|}{$1 / d^{2} \times 10^{4}$} & \multirow[b]{2}{*}{ h } & \multirow[b]{2}{*}{$\mathrm{k}$} & \multirow[b]{2}{*}{1} & \multirow[b]{2}{*}{$\mathrm{I} / \mathrm{I}_{\mathrm{O}}$} & \multicolumn{2}{|c|}{$1 / d^{2} \times 10^{4}$} & \multirow[b]{2}{*}{$\mathrm{h}$} & \multirow[b]{2}{*}{$\mathrm{k}$} & \multirow[b]{2}{*}{1} \\
\hline & obsd. & calc. & & & & & obsd. & calc. & & & \\
\hline $\mathrm{s}$ & 390 & 389 & 1 & $\overline{0}$ & 0 & $\mathrm{~m}$ & 5910 & 5898 & 2 & 1 & 6 \\
\hline $\mathrm{m}$ & 438 & 439 & 0 & 0 & 2 & $w$ & 6219 & 6217 & 4 & 0 & 0 \\
\hline vs & 780 & 777 & 1 & 1 & 0 & $\mathrm{~m}$ & 7049 & 7045 & 4 & 1 & 2 \\
\hline vs & 831 & 828 & 1 & 0 & 2 & vVw & 7456 & 7453 & 3 & 0 & 6 \\
\hline vvw & 957 & & & & & $\mathrm{vw}$ & 7794 & 7798 & 3 & 2 & 5 \\
\hline vrw & 1028 & & & & & vvw & 7981 & 7975 & 4 & 0 & 4 \\
\hline vrw & 1164 & & & & & vvw & 8579 & 8586 & 2 & 0 & 8 \\
\hline vw & 1218 & 1217 & 1 & 1 & 2 & vvw & 8772 & 8761 & 4 & 2 & 3 \\
\hline $\mathbf{s}$ & 1562 & 1554 & 2 & 0 & 0 & $\mathrm{vw}$ & 9000 & 9007 & 3 & 2 & 6 \\
\hline $\mathbf{S}$ & 1772 & 1766 & 1 & 1 & 3 & vw & 9525 & 9530 & 4 & 2 & 4 \\
\hline $\mathrm{vvw}$ & 1938 & 1943 & 2 & 1 & 0 & $\mathrm{w}$ & 10126 & 10103 & 5 & 1 & 0 \\
\hline vvw & 1999 & 1994 & 2 & 0 & 2 & $\mathrm{vw}$ & 10548 & 10543 & 5 & 1 & 2 \\
\hline vvw & 2149 & 2146 & 1 & 0 & 4 & vvw & 10920 & 10918 & 3 & 1 & 8 \\
\hline vvw & 2236 & & & & & vvw & 11396 & 11379 & 5 & 2 & 1 \\
\hline vs & 2390 & 2382 & 2 & 1 & 2 & $\mathrm{vw}$ & 11679 & 11708 & 5 & 2 & 2 \\
\hline $\mathrm{s}$ & 2546 & 2543 & 2 & 0 & 3 & vw & 11835 & 11861 & 5 & 1 & 4 \\
\hline$s$ & 3120 & 3109 & 2 & 2 & 0 & vvw & 12931 & 12930 & 2 & 1 & 10 \\
\hline s & 3323 & 3312 & 2 & 0 & 4 & vvw & 12946 & 12930 & 2 & 1 & 10 \\
\hline vvw & 3569 & 3548 & 2 & 2 & 2 & vvw & 13195 & 13212 & 5 & 3 & 0 \\
\hline vvw & 3704 & 3701 & 2 & 1 & 4 & vvw & 13207 & 13212 & 5 & 3 & 0 \\
\hline $\mathrm{s}$ & 3891 & 3886 & 3 & 1 & 0 & $\mathrm{vw}$ & 13640 & 13638 & 4 & 1 & 8 \\
\hline $\mathrm{s}$ & 3945 & 3937 & 3 & 0 & 2 & vvw & 13959 & 13951 & 3 & 2 & 9 \\
\hline $\mathrm{m}$ & 4354 & 4344 & 1 & 0 & 6 & vvw & 14452 & 14429 & 6 & 0 & 2 \\
\hline vvw & 4750 & 4732 & 1 & 1 & 6 & vw & 14802 & 14803 & 4 & 2 & 8 \\
\hline $\mathrm{m}$ & 4872 & 4867 & 2 & 2 & 4 & $\mathrm{vw}$ & 14808 & 14803 & 4 & 2 & 8 \\
\hline vvw & 5053 & 5052 & 3 & 2 & 0 & vvw & 14960 & 14970 & 5 & 3 & 4 \\
\hline vvw & 5275 & 5255 & 3 & 0 & 4 & vvw & 14981 & 14970 & 5 & 3 & 4 \\
\hline $\mathrm{m}$ & 5499 & 5491 & 3 & 2 & 2 & vvw & 15176 & 15181 & 4 & 4 & 5 \\
\hline $\mathrm{m}$ & 5654 & 5644 & 3 & 1 & 4 & & & & & & \\
\hline
\end{tabular}

\subsubsection{Preparation of $\mathrm{AgFBiF}_{6}$}

This material was synthesized in a multitude of ways: by oxidation of $\mathrm{AgBiF}_{6}$ with $F_{2}$, by metathesis between $\mathrm{AgFBF}_{4}$ and $\mathrm{KBiF}_{6}$, by an attempted oxidation of 
$\mathrm{AgFBF} 4$ using $\mathrm{O}_{2} \mathrm{BiF}_{6}$ as the oxidizer, and by an acid displacement reaction with $\mathrm{AgFBF}_{4}$. The acid displacement preparation yielded the most crystalline material, and so is described here. A passivated FEP T-reactor was loaded with $\mathrm{AgFBF}_{4}(97 \mathrm{mg}, 0.45$ $\mathrm{mmol})$ in one arm and $\mathrm{BiF}_{5}(128 \mathrm{mg}, 0.42 \mathrm{mmol})$ in the other. $\sim 2 \mathrm{~mL}$ aHF was condensed onto the $\mathrm{BiF}_{5}$ at $-196^{\circ} \mathrm{C}$ and then warmed to room temperature. All of the $\mathrm{BiF}_{5}$ dissolved to give a colorless solution, which was then poured over onto the dry $\mathrm{AgFBF}_{4}$. There was immediate effervescence as a dark blue-gray solid precipitated from the solution. After 30 min of agitation, the HF solution was decanted and removed, leaving behind a yellow-brown solid. A photograph of the blue-gray material (180 mg yield) showed an unidentified, complex pattern very similar to one generated here previously ${ }^{15}$ by the interaction of $\mathrm{F}_{2}$ on $\mathrm{AgSbF}_{6}$ in aHF. Magnetic measurements on this material reveal the same temperature-independent paramagnetism associated with all of the other $\mathrm{AgF}^{+}$salts (see Figure 5.4). Taking the product to be $\mathrm{AgFBiF}_{6}$, the yield was $97 \%$. 


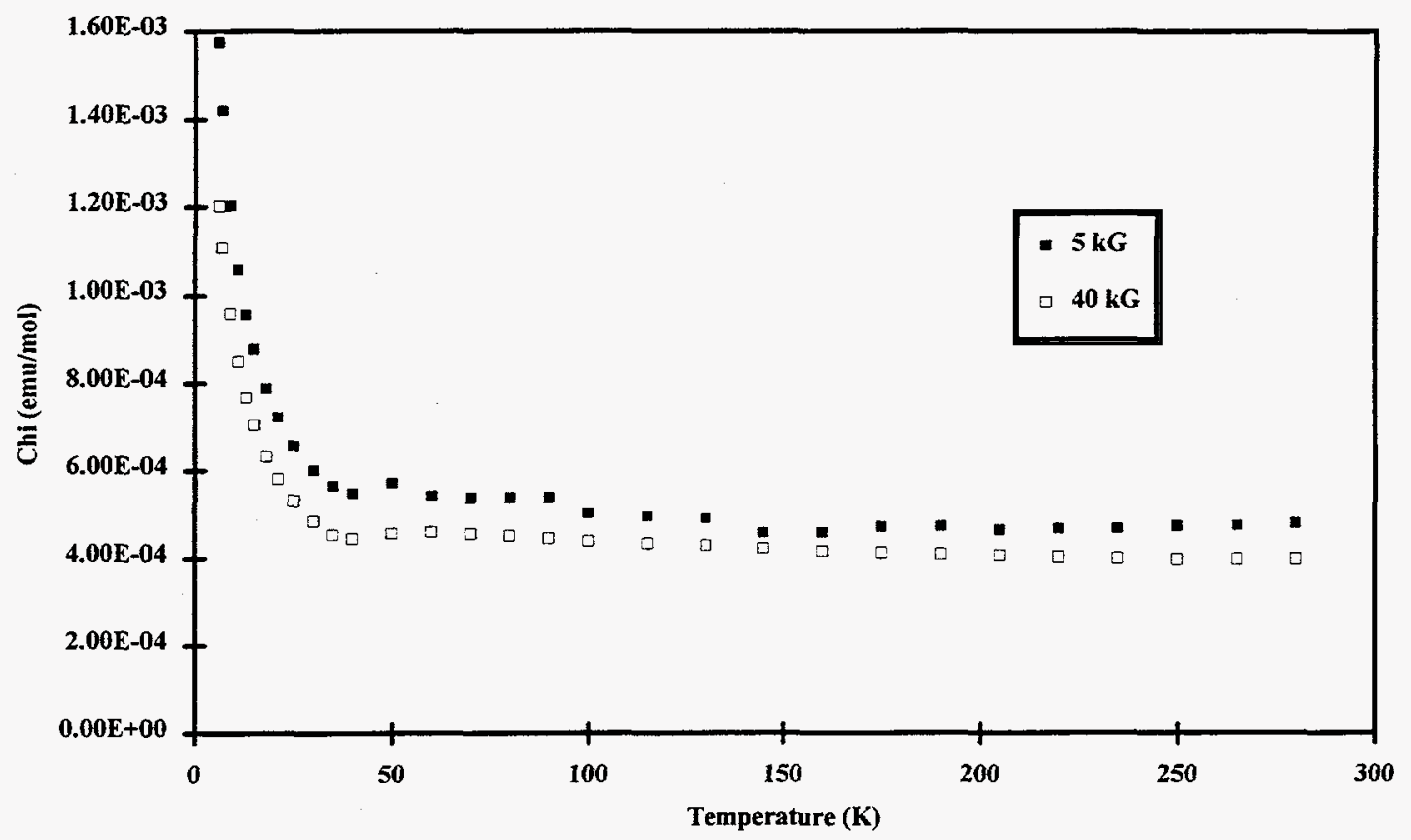

Figure 5.4. Magnetic data for $\mathrm{AgFBiF}_{6}$.

The field-dependence is likely an artifact of the instrumentation or due to a trace of a ferromagnetic impurity. 


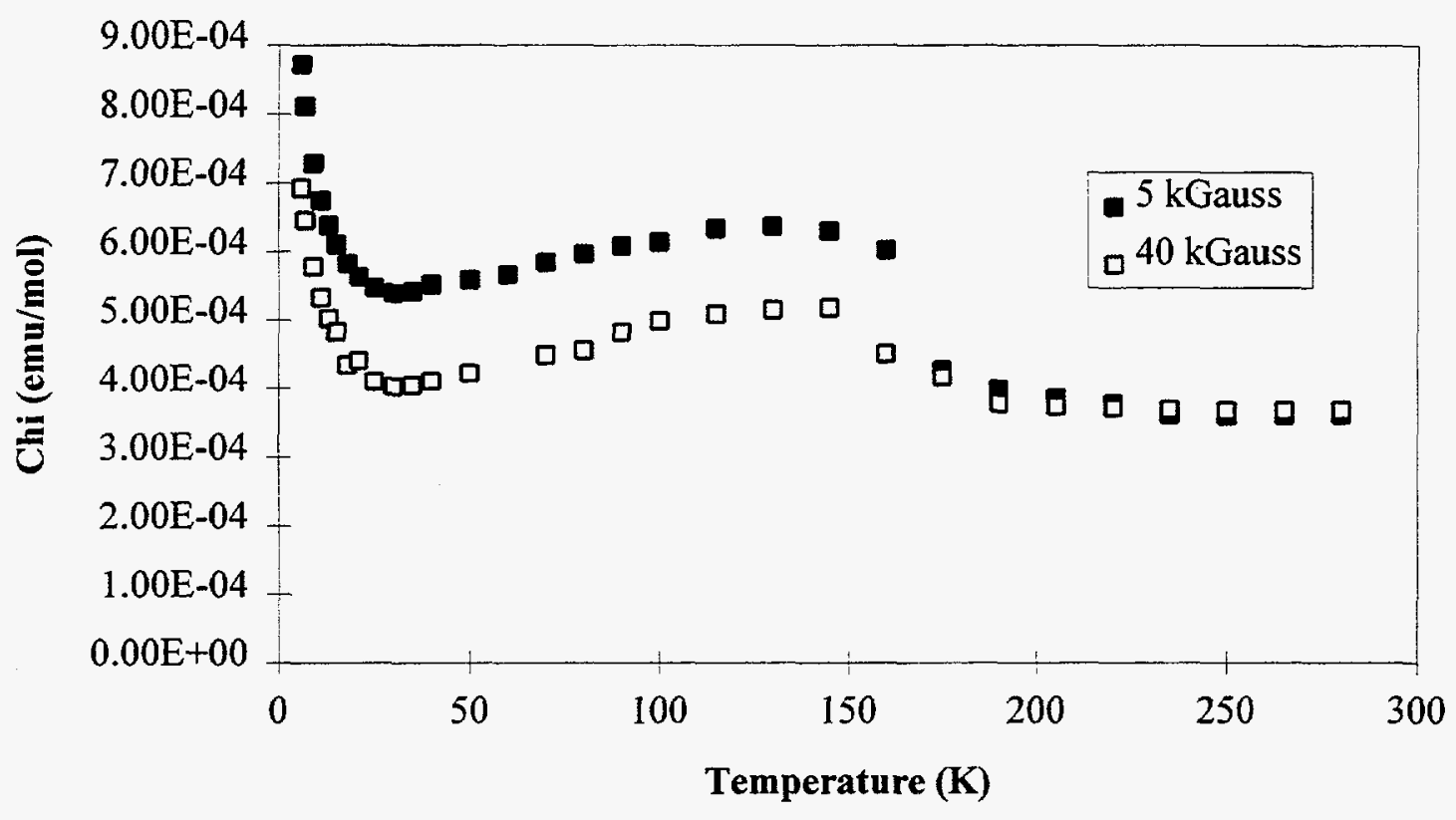

Figure 5.5. Magnetic data for $\mathrm{AgFSbF}_{6}$.

The field-dependence below $160 \mathrm{~K}$ is due to a small quantity of $\mathrm{AgF}_{2}$ present as impurity.

\subsubsection{Preparation of $\mathrm{AgRuF}_{6}$}

A passivated FEP T-reactor was loaded with AgF (311 mg, $2.45 \mathrm{mmol}$ ) in one arm and $\mathrm{RuF}_{5}(409 \mathrm{mg}, 2.08 \mathrm{mmol}$ ) in the other. Approximately $1.5 \mathrm{~mL}$ aHF was condensed onto the AgF, dissolving it completely at room temperature. . This solution was poured onto the dry $\mathrm{RuF}_{5}$. Reaction was immediately evident as the green $\mathrm{RuF}_{5}$ was replaced by an orange solid in less than a minute. Agitation of the reaction mixture was maintained for $5 \mathrm{~h}$, during which the product became a homogeneous orange solid. The solid was washed by decantation of the aHF solution, the aHF being back-distilled. 
Three more such washings were performed prior to removal of the HF. The orange product ( $650 \mathrm{mg}, 97 \%$ yield $\mathrm{AgRuF}_{6}$ ) was dried for $3 \mathrm{~h}$. An X-ray photograph of material prepared in this manner confirmed the identity of the product (tetragonal ${ }^{14}, a_{0}=$ $\left.4.85, c_{0}=9.54 \AA\right)$. The temperature dependence of the magnetic susceptibility of the orange solid is that of a simple paramagnet (see Figure 5.6). Because $\mathrm{AgRuF}_{6}$ has shown signs of photosensitivity, FEP storage tubes containing the solid were wrapped in aluminum foil.

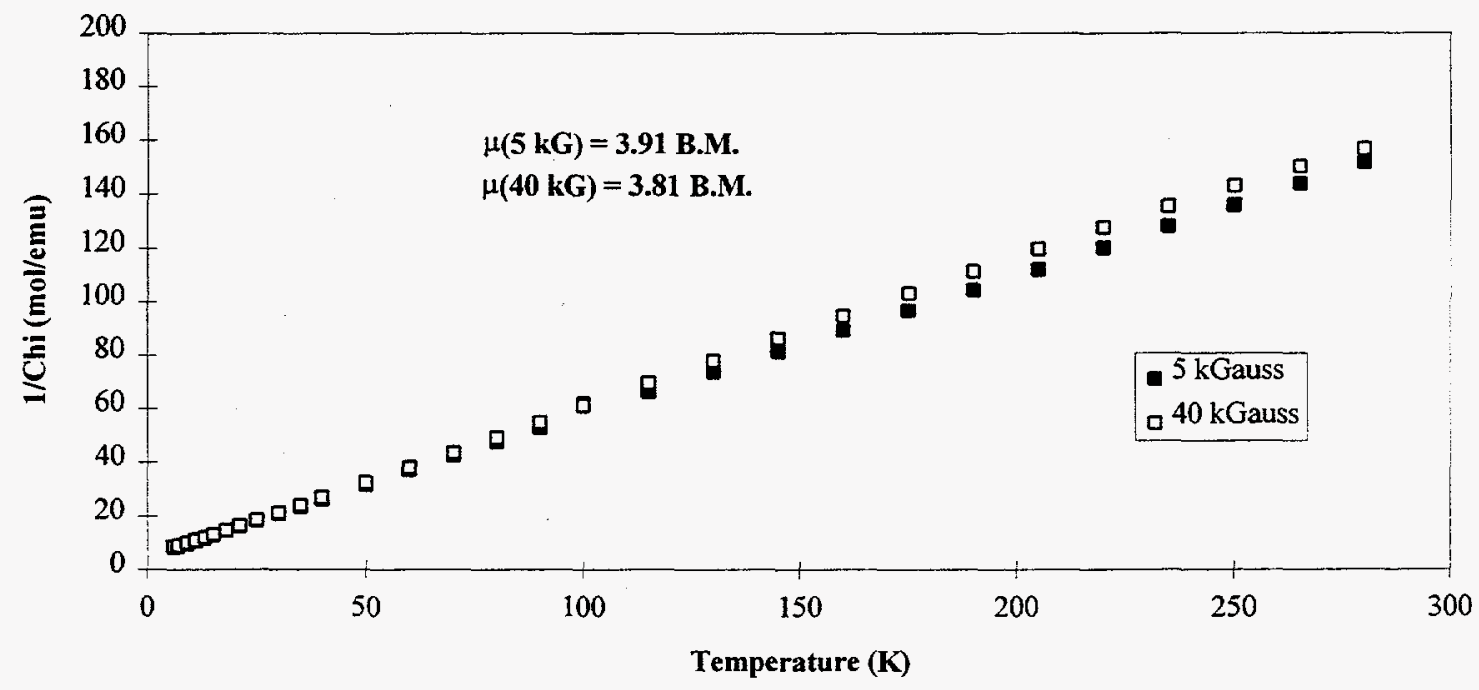

Figure 5.6. Magnetic data for $\mathrm{AgRuF}_{6}$, indicating a simple three-electron paramagnet.

\subsubsection{Preparation of $\mathrm{AgFRuF}_{6}$}

A passivated FEP T-reactor was loaded with $\mathrm{AgRuF}_{6}(247 \mathrm{mg}, 0.765 \mathrm{mmol})$.

After condensing $1.5 \mathrm{~mL}$ aHF onto the orange solid, the $\mathrm{HF}$ was warmed to room 
temperature. No color was visible in the solution as all the orange solid appeared to remain undissolved. The reactor was pressurized to 1500 torr with $\mathrm{F}_{2}$. Aluminum foil was used to wrap the arm of the reactor because of the suspected light sensitivity of $\mathrm{AgRuF}_{6}$. Continuous mechanical agitation was maintained for $19 \mathrm{~h}$, after which most of the $\mathrm{F}_{2}$ was removed by pumping the reactor down to 800 torr at room temperature. $\mathrm{BF}_{3}$ was then used to pressurize the reactor to 1400 torr. After less than a minute of agitation, the solution above the black product became very slightly blue. This solution was decanted and the product dried for $3 \mathrm{~h}$ under dynamic vacuum. An X-ray photo of the black product showed an XRPP unlike any other $\mathrm{AgF}^{+}$salt and indicated the presence of no known impurity. Gravimetry suggested that the material may be $\mathrm{AgFRuF}_{6}(257 \mathrm{mg}$, $98 \%$ yield). The magnetic susceptibility of the product is shown in Figure 5.7 in a CurieWeiss plot. Considering the susceptibility data from $50 \mathrm{~K}$ up to $280 \mathrm{~K}$, magnetic moments of 3.98 B.M. at $5 \mathrm{kGauss}$ and 3.87 B.M. at $40 \mathrm{kGauss}$ are calculated, corresponding very well with the spin-only value of 3.87 B.M. for a 3-electron paramagnet. These values are in good agreement with the $\mathrm{Ag}(\mathrm{I})$ salt $\mathrm{AgRuF}_{6}$, whose magnetic susceptibility data are shown in Figure 5.6. This result suggests that the $d^{3}$ $\mathrm{Ru}(\mathrm{V})$ anion dominates the magnetic signal, and the $d^{9} \mathrm{Ag}(\mathrm{II})$ lends an insignificant contribution to the overall susceptibility. 


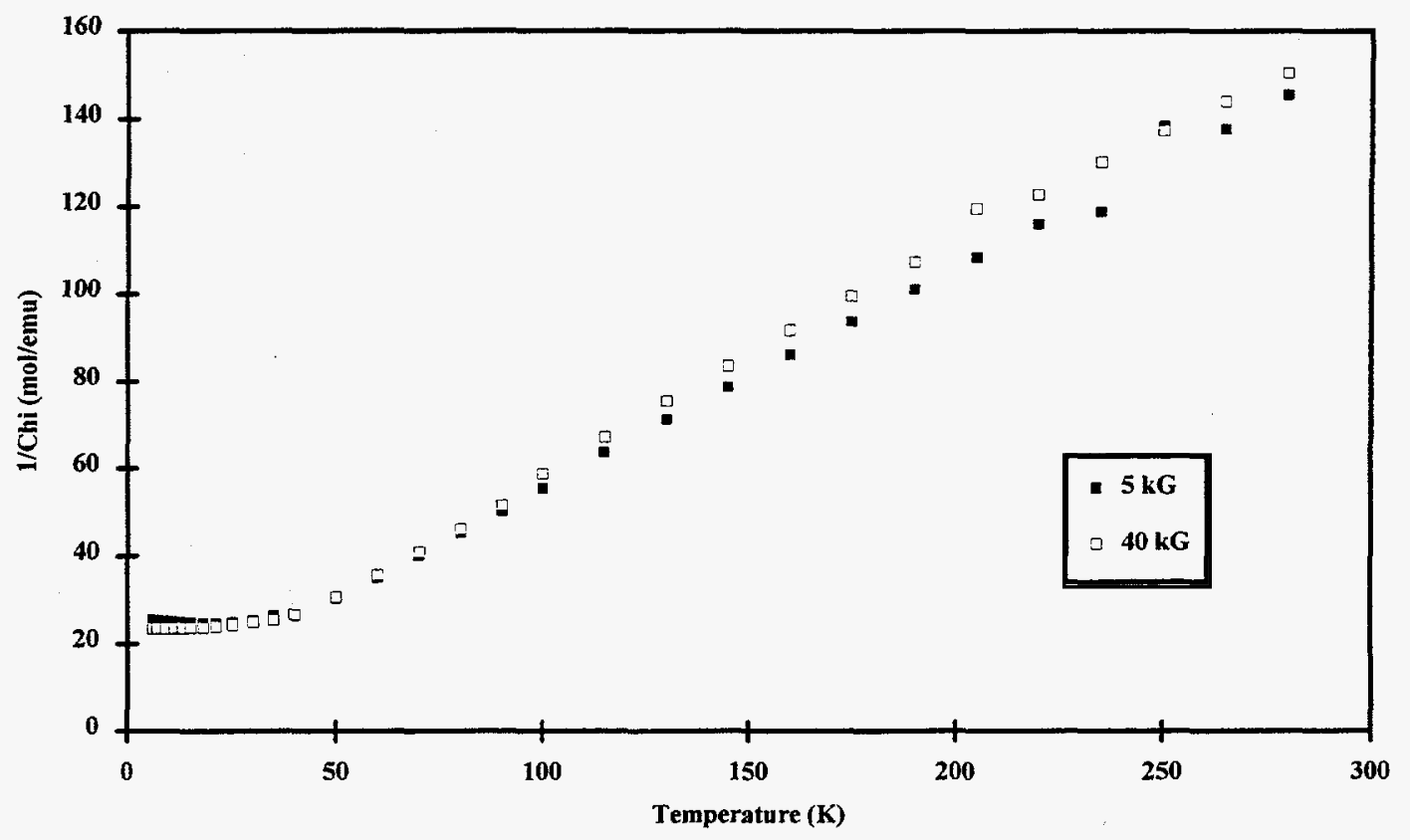

Figure 5.7. Magnetic data for $\mathrm{AgFRuF}_{6}$.

\subsubsection{Preparation of $\mathrm{AgOsF}_{6}$}

See the synthesis of $\mathrm{AgIrF}_{6}$ below in which reaction details are identical, the only exception being that $\mathrm{AgOsF}_{6}$ is a white solid. Representative weights are as follows: recrystallized $\mathrm{AgF}$ reagent $(104 \mathrm{mg}, 0.820 \mathrm{mmol}), \mathrm{SF}_{3} \mathrm{OsF}_{6}$ prepared as described in Chapter 3 (264 mg, $0.671 \mathrm{mmol}$ ), AgOsF 6 product ( $265 \mathrm{mg}, 96 \%$ yield). The tetragonal unit cell $\left(a_{0}=4.92, c_{0}=9.58 \AA\right)$ is described by Kemmitt et al. ${ }^{14}$

\subsubsection{Attempted Preparation of $\mathrm{AgFOsF}_{6}$}

Two synthetic routes were investigated for the production of $\mathrm{AgFOsF}_{6}$; the first was an oxidation reaction, and the second a metathetical attempt. 
A passivated FEP T-reactor containing $\mathrm{AgOsF}_{6}(118 \mathrm{mg}, 0.286 \mathrm{mmol})$ and about $2 \mathrm{~mL}$ aHF in one tube was pressurized with 2 atm $\mathrm{F}_{2}$ gas. Formation of the colorless gas $\mathrm{OsF}_{6}$ was not evident at room temperature. However, upon cooling the other arm of the reactor to $-196^{\circ} \mathrm{C}$, the $\mathrm{OsF}_{6}$ condensed over as a yellow solid. $\mathrm{SF}_{4}$ was introduced and slowly reacted to form $\mathrm{SF}_{3} \mathrm{OsF}_{6}$ by the reaction express in Equation (1). In this

$$
3 \mathrm{SF}_{4(\mathrm{~g})}+2 \mathrm{OsF}_{6(\mathrm{~g})} \stackrel{\mathrm{aHF}}{\longrightarrow} 2 \mathrm{SF}_{3} \mathrm{OsF}_{6(\text { solv })}+\mathrm{SF}_{6(\mathrm{~g})}
$$

experiment, the yield $\left(70 \mathrm{mg} \mathrm{SF}_{3} \mathrm{OsF}_{6}\right)$ based on Os metal was $62 \%$ and the formation of $\mathrm{AgF}_{2}$ as a byproduct was confirmed with $\mathrm{X}$-ray powder photography. Unreacted $\mathrm{AgOsF}_{6}$ also showed up as a minor component in the photograph.

The two powders $\mathrm{AgFBF}_{4}(110 \mathrm{mg}, 0.513 \mathrm{mmol})$ and $\mathrm{KOsF}_{6}(89 \mathrm{mg}, 0.26 \mathrm{mmol})$ were placed into the same tube of a passivated FEP T-reactor inside the DRILAB. After evacuation of the argon from the reactor, approximately $1.2 \mathrm{~mL}$ aHF was condensed onto the solid mixture at $-196^{\circ} \mathrm{C}$ and left to warm to room temperature. Reaction was evident even below room temperature as the blue $\mathrm{AgFBF}_{4}$ solid diminished in quantity, giving way to a brown precipitate. After $10 \mathrm{~min}$ of intermittent agitation at room temperature, no blue solid remained, so the volatile species were condensed into the other arm of the reactor at $-196^{\circ} \mathrm{C}$. Upon condensation, a deep yellow color was observed, indicative of $\mathrm{OsF}_{6}$. Approximately $1.5 \mathrm{mmol} \mathrm{SF}_{4}$ was condensed onto the frozen volatile species and then warmed to ca. $0^{\circ} \mathrm{C}$. Repeated thermal cycling of this mixture, followed by $1 \mathrm{~h} 10$ min at room temperature consumed all the $\mathrm{OsF}_{6}\{$ see Equation (1)\}, as revealed by the 
absence of yellow color upon freezing. The reactor was then evacuated, removing the aHF and any gases present, which resulted in the precipitation of a lavender solid. X-ray powder photography confirmed the identity of the product to be $\mathrm{SF}_{3} \mathrm{OsF}_{6}\left\{\right.$ cubic $^{16}, \mathrm{a}_{0}=$ $11.162(4) \AA$ \} , and the yield was $65 \mathrm{mg}, 64 \%$.

\subsubsection{Preparation of $\mathrm{AgIrF}_{6}$}

AgF (118 mg, $0.931 \mathrm{mmol}$ ) which had been recrystallized from anhydrous HF was loaded into one tube of a passivated FEP T-reactor inside the DRILAB. $\mathrm{SF}_{3} \operatorname{IrF}_{6}(241$ $\mathrm{mg}, 0.610 \mathrm{mmol}$ ), prepared as described in Chapter 3, was placed into the other tube of the reactor. After removing the argon from the reactor, about $3 / 4 \mathrm{~mL}$ aHF was condensed onto each of the reagents. Upon warming to room temperature, both of the powders dissolved completely to give colorless solutions. As the $\mathrm{AgF}$ solution was slowly poured onto the $\mathrm{SF}_{3} \mathrm{IFF}_{6}$ solution, the reaction mixture bubbled and an orange solid precipitated out of solution. The product was washed in the aHF three times then dried under dynamic vacuum for $3 \mathrm{~h}$. The XRPP of material prepared in this manner indicates the presence of pure $\mathrm{AgIrF}_{6}$ (tetragonal ${ }^{14}, a_{0}=4.85, c_{o}=9.70 \AA$ ). The yield is essentially quantitative ( $248 \mathrm{mg}, 98 \%$ yield).

\subsubsection{Preparation of $\mathrm{AgFIrF}_{6}$}

A passivated FEP T-reactor was loaded with $\operatorname{AgIrF}_{6}(244.1 \mathrm{mg}, 0.5895 \mathrm{mmol})$ inside the DRILAB. After removing the argon under dynamic vacuum, approximately 1 $\mathrm{mL}$ aHF was condensed onto the reagent. There were no signs of dissolution of the $\mathrm{AgIrF}_{6}$ as the $\mathrm{HF}$ melted and warmed to room temperature. The reactor was then 
pressurized to 1500 torr with $F_{2}$ gas and continuous agitation of the reaction mixture was begun. Within a few minutes, there was a noticeable darkening of the bright orange solid. After $3 \mathrm{~h}$ of continuous agitation, no orange solid remained; instead, there was a black powder beneath a slightly yellow HF solution. Agitation was continued for another $3 \mathrm{~h}$ before liquid nitrogen was used to freeze the reaction mixture solid at $-196^{\circ} \mathrm{C}$. Any remaining $F_{2}$, having a vapor pressure of about 300 torr at this temperature, was then pumped away under dynamic vacuum. Once again, the HF was melted and warmed up to room temperature. Then the reactor was pressurized to 1500 torr with $\mathrm{BF}_{3}$ gas. This turned the HF solution from pale yellow to pale green, presumably due to the formation of the slightly soluble species $\mathrm{AgFBF}_{4}$. After washing the black product six times in the $\mathrm{HF}$, the solution still appeared green, so the HF was decanted and pumped away, leaving a brown residue in the other arm of the reactor. Temperature- and field-dependent magnetic susceptibility measurements of this sample reveal the presence of only a minute $\mathrm{AgF}_{2}$ impurity (see Figure 5.8). X-ray powder photographs of material prepared in this manner show only the XRPP of $\operatorname{AgFIrF}_{6}$ \{orthorhombic ${ }^{17}, a_{0}=7.627(2), b_{0}=7.068(2), c_{0}$ $=10.253(4) \AA\}$.

A plot of the magnetic susceptibility is provided in Figure 5.8. Once again, temperature-independence is observed, except in the low-temperature realm. In this case, a dip in the susceptibility, which has been observed in other $\mathrm{AgF}^{+}$samples, appears to be the result of impurity. In many other $\mathrm{AgF}^{+}$compounds, this dip was apparent in some of 
the magnetic measurements, but not reproducible from one sample preparation to the next. In this case, careful attention to the purity of the product was not successful in eliminating the dip below $\sim 65 \mathrm{~K}$ from these data, only minimizing it.

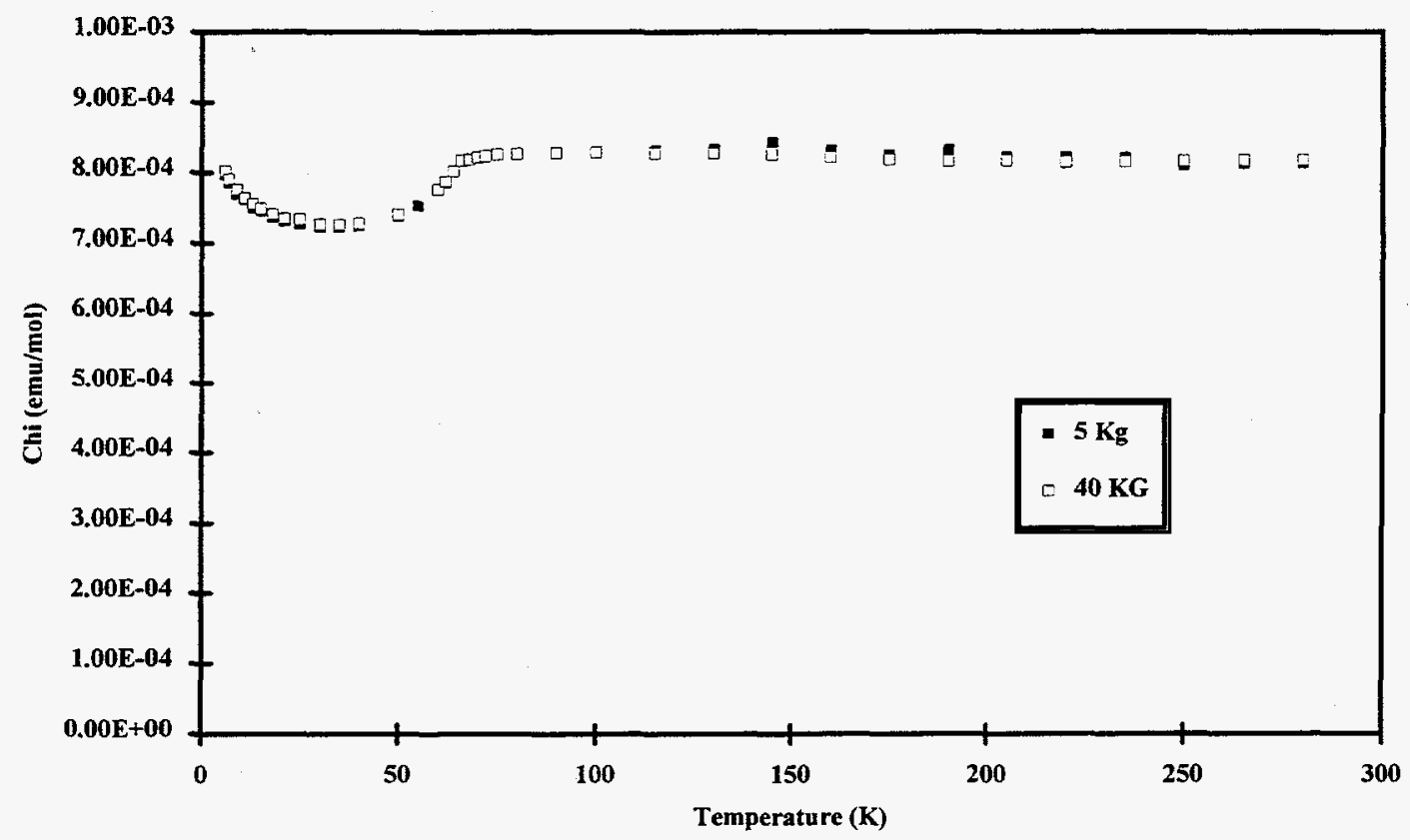

Figure 5.8. Magnetic data for $\mathrm{AgFIrF}_{6}$.

\subsection{Results and Discussion}

The early work on $\mathrm{AgF}^{+}$salts in these laboratories ${ }^{15}$ had included preparation of $\mathrm{AgF}^{+} \mathrm{BF}_{4}^{-}$and $\mathrm{AgF}^{+} \mathrm{SbF}_{6}^{-}$by fluorination of the $\mathrm{Ag}(\mathrm{I})$ salts in $\mathrm{aHF}$. These syntheses were so clean that they immediately invited extension of the approach to the general class. An additional advantage that this approach provided to the fluorination of the $\operatorname{Ag}(\mathrm{I})$ salt was the slowness of the oxidation since neither $\mathrm{Ag}^{+} \mathrm{A}_{\mathrm{L}} \mathrm{F}^{-}$salts (where $\mathrm{A}_{\mathrm{L}}$ is a Lewis acid) nor 
$F_{2}$ are very soluble in aHF. This proved to be fruitful in the production of highly crystalline $\mathrm{AgF}^{+}$salts.

To check on the chemical purity of the preparations, it was routine to fingerprint the material by its X-ray powder diffraction pattern, and these patterns were indexed by single crystal data wherever possible. In addition, the preparations were characterized by their magnetic properties, which, as revealed in the Experimental section above and discussed in Chapter 6, were highly characteristic for all $\mathrm{AgF}^{+}$salts. In all cases, the magnetic susceptibility of the cation exhibited temperature-independent paramagnetism at least above $50 \mathrm{~K}$. When field-dependent paramagnetism was observed, this was invariably associated with $\mathrm{AgF}_{2}$ impurity (ferromagnetic ${ }^{9} \mathrm{~T}_{\mathrm{c}}=163 \mathrm{~K}$ ). Except in the case of $\mathrm{AgFRuF}_{6}$, whose anion is a simple paramagnetic, simple paramagnetism would have indicated $\mathrm{Ag}\left(\mathrm{MF}_{6}\right)_{2}$ salts (see Chapter 6), but such impurities were not present in the preparations involving the fluorination of $\mathrm{Ag}^{+} \mathrm{A}_{\mathrm{L}} \mathrm{F}^{-}$salts.

The experimental findings indicate that there are two important limitations to the existence of $\mathrm{Ag}^{+} \mathrm{A}_{\mathrm{L}} \mathrm{F}^{-}$salts. One is due to the high fluoro-acidity of the cation, which can abstract $\mathrm{F}^{-}$from less strong acids, $\mathrm{A}_{\mathrm{L}}$. And the other (which is observed particularly with certain transition-element $\mathrm{A}_{\mathrm{L}}$ ) arises from the high electron affinity of $\mathrm{AgF}^{+}$, which can bring about electron oxidation of the anion.

The weak fluoro-acidity of $\mathrm{PF}_{5}$ is responsible for the failure to prepare $\mathrm{AgFPF}_{6}$ by fluorination of $\mathrm{AgPF}_{6}$, this reaction proceeding ${ }^{17}$ as follows: 


$$
\mathrm{AgPF}_{6}+1 / 2 \mathrm{~F}_{2} \stackrel{\mathrm{aHF}}{\longrightarrow} \mathrm{AgF}_{2}+\mathrm{PF}_{5}
$$

Here, $\mathrm{PF}_{6}^{-}$is seen to be an effective $\mathrm{F}^{-}$donor even to $\mathrm{AgF}^{+}$. Although the $\mathrm{F}^{-}$affinity of $\mathrm{BF}_{3(\mathrm{~g})}$ is approximately $10 \mathrm{kcal} \cdot \mathrm{mol}^{-1}$ less than that of $\mathrm{PF}_{5(\mathrm{~g})}$, the greater lattice energy of small-cation $\mathrm{BF}_{4}^{-}$salts more than compensates for that deficiency. This probably accounts for the existence of $\mathrm{AgFBF}_{4}$ and the failure to prepare $\mathrm{AgFPF}_{6}$.

Some of the acids (e.g. AsF 5 ) whose conjugate bases make up the anions (e.g. $\mathrm{AsF}_{6}^{-}$) of the $\mathrm{AgF}^{+}$salts have a considerable HF-affinity, as gauged by their solubility in it. The affinity of $\mathrm{A}_{\mathrm{L}}$ for aHF, working in concert with the high $\mathrm{F}^{-}$affinity of the $\mathrm{AgF}^{+}$ cation, probably explains the solvolysis of certain $\mathrm{AgF}^{+} \mathrm{A}_{\mathrm{L}} \mathrm{F}^{-}$salts when they are immersed in a large quantity of aHF. Then the reaction is essentially as expressed in Equation (4):

$$
\mathrm{AgF}^{+} \mathrm{A}_{\mathrm{L}} \mathrm{F}^{-} \stackrel{\mathrm{aHF}}{\rightleftharpoons} \mathrm{AgF}_{2}+\mathrm{A}_{\mathrm{L}}(\mathrm{HF})_{\mathrm{n}}
$$

This solvolysis is not observed when $A_{\mathrm{L}}$ has only a weak HF-affinity, as in the case of $\mathrm{AgFIrF}_{6}$, for which $\mathrm{A}_{\mathrm{L}}$ (i.e. $\operatorname{IrF}_{5}$ ) is insoluble in aHF. Because Equation (4) represents an equilibrium, additional acid may be introduced to avoid $\mathrm{AgF}_{2}$ formation and ensure pure $\mathrm{AgFA}_{\mathrm{L}} \mathrm{F}$ product. However, in such preparations, $\mathrm{Ag}(\mathrm{II})$ is always sacrificed to $\operatorname{Ag}\left(\mathrm{A}_{\mathrm{L}} \mathrm{F}\right)_{2}$ formation (at least in solution) by the following equilibrium:

$$
\mathrm{AgF}^{+} \mathrm{A}_{\mathrm{L}} \mathrm{F}^{-}+\mathrm{A}_{\mathrm{L}} \stackrel{\mathrm{aHF}}{\rightleftharpoons} \mathrm{Ag}_{\text {solv }}^{\mathrm{II}}+2 \mathrm{~A}_{\mathrm{L}} \mathrm{F}_{\text {solv }}^{-}
$$

Attempts to synthesize $\mathrm{AgFOsF}_{6}$ from the $\mathrm{Ag}(\mathrm{I})$ salt and molecular fluorine all met with failure, generating $\mathrm{OsF}_{6}$ instead. Treatment of $\mathrm{KOsF}_{6}$ or $\mathrm{LiOsF}_{6}$ in aHF with 
fluorine gas does not liberate $\mathrm{OsF}_{6}$. Consequently, it may be surmised that the mechanism which leads to the release of $\mathrm{OsF}_{6}$ from its anion involves the initial generation of a $\mathrm{Ag}(\mathrm{II})$ species, perhaps proceeding as follows:

$$
\begin{gathered}
\mathrm{Ag}^{+}+\mathrm{F}-\mathrm{F} \stackrel{\mathrm{aHF}}{\longrightarrow} \mathrm{AgF}^{+}+\mathrm{F}^{\cdot} \\
\mathrm{AgF}^{+}+\mathrm{OsF}_{6}^{-} \stackrel{\mathrm{aHF}}{\longrightarrow} \mathrm{AgF}+\mathrm{OsF}_{6}
\end{gathered}
$$

The $\mathrm{AgF}$ generated in this scenario is subsequently oxidized to $\mathrm{AgF}_{2}$ by the fluorine gas present, and the fluorine radical may oxidize yet another $\operatorname{Ag}(\mathrm{I})$. There is, however, another possible mechanism in which the silver only indirectly assists in the oxidation of the osmium(V):

$$
\begin{gathered}
\mathrm{Ag}^{+}+\mathrm{F}-\mathrm{F} \stackrel{\mathrm{aHF}}{\longrightarrow} \mathrm{AgF}^{+}+\mathrm{F}^{\cdot} \\
\mathrm{F}^{\cdot}+\mathrm{OsF}_{6}^{-} \stackrel{\mathrm{aHF}}{\longrightarrow}(\mathrm{HF})_{\mathrm{n}} \mathrm{F}^{-}+\mathrm{OsF}_{6}
\end{gathered}
$$

In this scenario, the solvated $\mathrm{AgF}^{+}$and $\mathrm{F}^{-}$quickly combine to form $\mathrm{AgF}_{2}$. Reaction via this pathway does not suggest that polymeric $(\mathrm{AgF})_{n}^{\mathrm{n}+}$ has sufficient electron affinity to affect the oxidation of $\mathrm{OsF}_{6}^{-}$. Indeed, the only way to truly test such a hypothesis is to avoid the use of molecular fluorine altogether. For this reason, the metathetical synthetic approach was attempted, and produced essentially the same results:

$$
\mathrm{KOsF}_{6}+\mathrm{AgFBF}_{4} \rightarrow \mathrm{KBF}_{4}+\mathrm{AgF}+\mathrm{OsF}_{6}
$$

thus confirming that the $(\mathrm{AgF})_{\mathrm{n}}^{\mathrm{n}+}$ cation does indeed have the electron-withdrawing potential required to generate $\mathrm{OsF}_{6}$ from its monoanion. 
In contrast to the osmium reaction, $\mathrm{AgFIrF}_{6}$ was readily prepared by the oxidation of $\mathrm{AgIrF}_{6}$ with molecular $\mathrm{F}_{2}$ in aHF.

$$
\mathrm{AgIrF}_{6}+1 / 2 \mathrm{~F}_{2} \stackrel{\mathrm{aHF}}{\longrightarrow} \mathrm{AgFIrF}_{6}
$$

This synthesis is particularly interesting because although the $(\mathrm{AgF})_{\mathrm{n}}^{\mathrm{n}+}$ cation forms a stable complex with the hexafluoroiridate $(\mathrm{V})$ anion, evidence is provided in Chapter 7 for the oxidation of this anion by solvated $\mathrm{Ag}(\mathrm{II})$. Indeed, it is interesting to note that $\mathrm{AgFIrF}_{6}$ can exist only in a narrow $\mathrm{pH}$ range in the aHF. Acidification of the solution with an appropriately strong Lewis acid $\left(A_{L}\right)$ removes the fluoride from the $\mathrm{AgF}^{+}$unit, freeing the $\mathrm{Ag}(\mathrm{II})$ into solution and permitting oxidation of $\operatorname{IrF}_{6}^{-}$to $\operatorname{IrF}_{6}$.

$$
\mathrm{AgFIFF}_{6}+\mathrm{A}_{\mathrm{L}} \stackrel{\mathrm{aHF}}{\longrightarrow} \mathrm{Ag}_{\text {(solv) }}^{\mathrm{II}}+\mathrm{IrF}_{6}^{-}+\mathrm{A}_{\mathrm{L}} \mathrm{F}^{-} \stackrel{\mathrm{aHF}}{\longrightarrow} \mathrm{AgA}_{\mathrm{L}} \mathrm{F}_{(\mathrm{s})}+\mathrm{IrF}_{6(\mathrm{~g})}
$$

Furthermore, addition of a fluoro-base to the aHF results in the formation of $\mathrm{AgF}_{2}$ and the dissolution of the $\mathrm{IrF}_{6}^{-}$counterion.

$$
\mathrm{AgFIrF}_{6}+\mathrm{F}^{-} \stackrel{\mathrm{aHF}}{\longrightarrow} \mathrm{AgF}_{2(\mathrm{~s})}+\mathrm{IrF}_{6(\text { (solv })}^{-}
$$

While any concentration of base in the aHF is sufficient to cause the decomposition of $\mathrm{AgFIrF}_{6}$, it was observed that a weak fluoro-acid such as $\mathrm{BF}_{3}$ does not have the fluoride ion affinity required to abstract the fluoride from $\mathrm{AgF}^{+}$to a significant extent. Consequently, it was possible to obtain $\mathrm{AgFIrF}_{6}$ essentially free of $\mathrm{AgF}_{2}$ by "washing" it in a solution of $\mathrm{aHF} / \mathrm{BF}_{3}$. This washing procedure first converts any $\mathrm{AgF}_{2}$ to $\mathrm{AgFBF}_{4}$ and then dissolves it, generating a slightly blue-tinted solution, probably $\mathrm{Ag}^{2+}\left(\mathrm{BF}_{4}^{-}\right)_{2}$ as 
fully solvated ions. Since the $\mathrm{BF}_{3}$ does not promote significant dissolution of $\mathrm{AgFIrF}_{6}$, the absence of $\mathrm{AgF}_{2}$ was manifest in a colorless solution above the black product.

\subsection{Conclusions}

Fluorination of $\mathrm{Ag}(\mathrm{I})$ salts in aHF has been found to be an appropriate method for the preparation of $\mathrm{AgF}^{+}$salts, in some cases yielding single crystals of sufficient quality for X-ray diffraction studies. Two considerations have been found to be important for the stability of a given $\mathrm{AgFA}_{L} F$ salt: the fluoride affinity of $A_{L}$ and, for transition-element

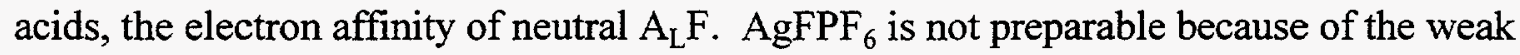
$\mathrm{F}^{-}$affinity of $\mathrm{PF}_{5}$. And yet, this compound must be very close to thermodynamic stability because $\mathrm{AgFBF}_{4}$ is preparable.

Oxidation of $\mathrm{OsF}_{6}^{-}$is easily achieved at room temperature by $(\mathrm{AgF})_{\mathrm{n}}^{\mathrm{n}+}$ because the electron affinity of $A_{L} F$ in this case (i.e. $\mathrm{OsF}_{6}$ ) is not high enough to take the electron from $\mathrm{Ag}(\mathrm{I})$. This has been demonstrated by oxidative fluorination reactions of $\mathrm{AgOsF}_{6}$, as well as by metathetical combination of an alkali $\mathrm{OsF}_{6}^{-}$salt with another $\mathrm{AgF}^{+}$salt in aHF solution. AgFIrF 6 , however, is readily prepared in high purity in aHF solution. Therefore, on the graded scale provided by the electron affinities of the third transition series metal hexafluorides, $(\mathrm{AgF})_{\mathrm{n}}^{\mathrm{n}+}$ lies somewhere between $\mathrm{OsF}_{6}$ and $\mathrm{IrF}_{6}$ in oxidizing potential. 


\subsection{References}

${ }^{1}$ B. Frlec, D. Gantar, and J. H. Holloway, J. Fluorine Chem., 20 (1982), pp 385-396.

${ }^{2}$ B. Žemva, K. Lutar, A. Jesih, W. J. Casteel, Jr., A. P. Wilkinson, D. E. Cox, R. B. Von Dreele, H. Borrmann, and N. Bartlett, J. Am. Chem. Soc., 113 (1991), pp 4192-4198.

${ }^{3}$ B. Žemva, R. Hagiwara, W. J. Casteel, Jr., K. Lutar, A. Jesih, and N. Bartlett, J. Am. Chem. Soc., 112 (1990), pp 4846-4849.

${ }^{4}$ W. J. Casteel, Jr., G. Lucier, R. Hagiwara, H. Borrmann, and N. Bartlett, J. Solid State Chem., 96 (1992), pp 84-96.

${ }^{5}$ W. J. Casteel, Jr., Ph. D. Thesis, (1992) Chapter 7, U.C. Berkeley.

${ }^{6}$ G. Lucier, C. Shen, W. J. Casteel, Jr., L. Chacón, and N. Bartlett, J. Fluorine Chem., (1995) in press.

${ }^{7}$ B. G. Müller, Angew. Chem., 99 (1987), p 685.

${ }^{8}$ A. Jesih, K. Lutar, B. Žemva, Z. Anorg. Allg. Chem., 588 (1990), pp 77-83.

${ }^{9}$ E. Gruner and W. Klemm, Naturwissenschaften, 25 (1937), p 59; and P. Charpin, A. J. Dianoux, H. Marquet-Ellis, and Nguyen-Nghi, C. R. Acad. Sci. Paris, 264 (1967), p 1108.

${ }^{10}$ B. N. Figgis, Introduction to Ligand Fields, (1966) New York: Interscience.

11 J. Clark, Z. Pistorius, Phys. Chem. Neue Folge. (Weisbaden), 88 (1974), p 242.

${ }^{12}$ D. E. Cox, J. Chem. Soc., (1956), p 876. 
${ }^{13}$ D. Gantar, B. Frlec, D. R. Russell, and J. H. Holloway, Acta Cryst., C43 (1987), pp 618-620.

${ }^{14}$ R. D. W. Kemmitt, D. R. Russell, and D. W. A. Sharp, J. Chem. Soc., 844 (1963), pp $4408-4413$.

${ }^{15}$ W. J. Casteel, Jr., Ph. D. Thesis, (1992) Chapter 6, U.C. Berkeley.

${ }^{16}$ N. K. Jha, Ph.D. Thesis, (1965) Chapter 2, U. British Columbia.

${ }^{17}$ G. Lucier, J. Munzenberg, W. J. Casteel, Jr., and N. Bartlett, Inorg. Chem., (1995) in press. 


\section{Chapter 6}

\section{Structure and Physical Properties of $\mathrm{AgF}^{+}$Salts}

\subsection{Introduction}

In 1987, Gantar et al. ${ }^{1}$ published the structure of $\mathrm{AgFAsF}_{6}$. Their single-crystal $\mathrm{X}$-ray study revealed that this $\mathrm{AgF}^{+}$salt contains a one-dimensional chain cation of formula $(\mathrm{AgF})_{\mathrm{n}}^{\mathrm{n+}}$ which is surrounded by nearly-octahedral $\mathrm{AsF}_{6}^{-}$species. Although this chain is kinked at the fluorine bridges, it is straight at the silver links, and the two Ag-F bond lengths are not significantly different. The tetrafluoroborate, which was first made in these laboratories ${ }^{2}$, was the next $\mathrm{AgF}^{+}$salt to be prepared. Its synthesis and $\mathrm{X}$-ray powder diffraction data were reported ${ }^{2}$ in 1990 . Analysis of the unit cell data suggested that, again, the $(\mathrm{AgF})_{\mathrm{n}}^{\mathrm{n}+}$ chain cation was present, this time constrained by the symmetry of the structure to be perfectly linear. A technique for the growth of single crystals of the $\mathrm{AgF}^{+}$salts was soon developed as part of the work described in this chapter. Single crystal X-ray data for $\mathrm{AgFBF}_{4}$ were collected, the resulting structure solution confirming ${ }^{3}$ the suspected structure. In this same publication, X-ray powder diffraction data for the 
tetrafluoroaurate(III) and hexafluoroaurate(V) salts were reported. $\mathrm{AgFAuF}_{4}$ was found to be isomorphous ${ }^{4}$ with $\mathrm{CuFAuF}_{4}$, and $\mathrm{AgFAuF}_{6}$ was seen to be isomorphous with $\mathrm{AgFAsF}_{6}$, both structures containing $(\mathrm{AgF})_{\mathrm{n}}^{\mathrm{n}+}$ chains kinked at the $\mathrm{F}$ bridge of the cation. With the size of the class of $\mathrm{AgF}^{+}$salts having increased to include four compounds of three distinct structures, it was interesting to note that all of these materials contained the chain cation with intrachain Ag-F bond lengths always very nearly $2.0 \AA$. This work has since expanded the $\mathrm{AgF}^{+}$class of compounds to include the hexafluoroiridate $(\mathrm{V})$ and hexafluororuthenate(V), whose structures have been solved and are discussed below, and the hexafluoroantimonate(V) and hexafluorobismuthate(V), single crystals of which have proven to be difficult to preserve.

It was recognized from the start of our investigations into the $\mathrm{AgF}^{+}$salts that the magnetic susceptibility data (provided in Chapter 5) are consistent with that to be expected of a metallic system. In fact, every known $\mathrm{AgF}^{+}$salt has exhibited the same temperature-independent, weak paramagnetism. Furthermore, polarized light transmission experiments performed on $\mathrm{AgFBF}_{4}$ are indicative of a one-dimensional electronic conductor. So it is reasonable to postulate that a partially-filled conduction band arises for the $(\mathrm{AgF})_{\mathrm{n}}^{\mathrm{n}+}$ chain cation, for which one-dimensional electronic conductivity is anticipated. Unfortunately, no electrical conductivity has been directly measured, perhaps owing to the extreme reactivity of the materials under investigation. 


\subsection{Structure of $\mathrm{AgFBF}_{4}$}

The preparation of bronze $\mathrm{AgFBF}_{4}$ almost unavoidably produced macroscopic crystals (see Experimental section of Chapter 5), one of which was selected for single crystal X-ray structure determination. Crystals of violet $\mathrm{AgFBF}_{4}$ were obtained by first placing a small amount ( $\sim 30-40 \mathrm{mg})$ of $\mathrm{AgBF}_{4}$ in a passivated FEP T-reactor constructed with $3 / 8$ " tubing. About $1 \mathrm{~mL}$ aHF was condensed onto the powder and then warmed to room temperature. The reactor was pressurized to $\sim 1000$ torr with $F_{2}$ gas and left to sit undisturbed. After $20 \mathrm{~h}$, there was no evidence of remaining $\mathrm{AgBF}_{4}$, and violet-blue crystals, mostly needles, were seen to exist in contact with a light blue solution. The aHF solution was decanted off and the aHF removed under dynamic vacuum prior to vacuumdrying the crystals for $3 \mathrm{~h}$.

Single crystal structure determinations of bronze and violet $\mathrm{AgFBF}_{4}$ showed no significant differences ${ }^{3}$, other than that the violet crystal provided a slightly higher precision. Details of the structure solution for the violet material are provided in Table 6.1. The structure was successfully refined in space group $P 4 / n$. The earlier supposition ${ }^{2}$ of a linear $(\mathrm{AgF})_{\mathrm{n}}^{\mathrm{n}+}$ chain, based on the tetragonal unit cell and tentative space group assignment, was confirmed. 
Table 6.1. Crystal Data and Details of the Structure Refinement of $\mathrm{AgFBF}_{4}$

\begin{tabular}{|c|c|}
\hline Formula, mol wt & $\mathrm{AgBF}_{5}, 213.67 \mathrm{amu}$ \\
\hline Temperature & $293 \mathrm{~K}$ \\
\hline$a_{o}$ & $6.7000(3) \AA$ \\
\hline$c_{o}$ & $4.0113(2) \AA$ \\
\hline$V$ & $180.07(1) \AA^{3}$ \\
\hline$Z$ & 2 \\
\hline Space group & P 4/n (No. 85) \\
\hline$d_{\text {calc }}$ & $3.940 \mathrm{~g} \cdot \mathrm{cm}^{-3}$ \\
\hline Crystal size & $0.08 \times 0.13 \times 0.35 \mathrm{~mm}$ \\
\hline Wavelength (Mo K $\alpha$ ) & $0.71073 \AA$ \\
\hline$\mu$ & $55.33 \mathrm{~cm}^{-1}$ \\
\hline Diffractometer & $\begin{array}{l}\text { Enraf-Nonius CAD4, } \\
\text { graphite monochromator }\end{array}$ \\
\hline Scan range & $3<2 \Theta<76^{\circ} ; \pm h, \pm k, l$ \\
\hline Scan angle & $1.1^{\circ}+0.35 \tan \Theta ; \omega / \Theta-\operatorname{scan}$ \\
\hline Scan speed & variable, $t_{\max }=80 \mathrm{sec}$ \\
\hline Data collected & 2166 \\
\hline Independent & $494 ; R_{\text {int }}=0.026$ \\
\hline Absorption correction & Empirical $\Psi$-scans, $\Delta \Psi=10^{\circ}, 8 \mathrm{hkl}$ \\
\hline Structure solution & $\begin{array}{l}\text { Based on packing considerations from } \\
\text { powder data }\end{array}$ \\
\hline Refinement & $\begin{array}{l}\text { Full matrix least squares, } \\
17 \text { parameters }\end{array}$ \\
\hline Weighting scheme & $1 / \sigma^{2}$ \\
\hline Extinction correction & $F_{\text {corr }}=F_{\text {obs }} \cdot(1+g \cdot I), g=1.60 \times 10^{-5}$ \\
\hline$R$ & 0.0193 \\
\hline$w R$ & 0.0250 \\
\hline Goodness-of-fit & 1.104 \\
\hline
\end{tabular}

The extended structure of $\mathrm{AgFBF}_{4}$ is illustrated in Figure 6.1. Four columns of linearly close-packed $\mathrm{BF}_{4}^{-}$are seen to surround each linear $(\mathrm{AgF})_{\mathrm{n}}^{\mathrm{n+}}$ chain, and vice versa. Each $\mathrm{Ag}(\mathrm{II})$ atom is square-coordinated by four ligands of the anions, each from one of the four surrounding $\mathrm{BF}_{4}^{-}$, and is almost in the same plane as these four $\mathrm{F}$ ligands. Interaction of the almost-ideally tetrahedral $\mathrm{BF}_{4}^{-}$with four nearest-neighbor $\mathrm{Ag}(\mathrm{II})$ 
species, via the $\mathrm{F}$ ligands, results in an alternation of the $\operatorname{Ag}(\mathrm{II}) z$ parameters in the four surrounding $(\mathrm{AgF})_{\mathrm{n}}^{\mathrm{n}+}$ columns, these $z$ parameters being in step with those of the $\mathrm{F}$ ligands of an anion. As illustrated in Table 6.4, the Ag--F interatomic distance between cation and each of the four nearest anion ligands is $2.330(2) \AA$. These are consistent with interionic contacts. The Ag--F distances within the chain, on the other hand, are short and must represent appreciable overlap of the valence-shell orbitals of the $\mathrm{Ag}(\mathrm{II})$ (e.g. $\left.4 d_{z} 2\right)$ and the $\mathrm{F}\left(\right.$ e.g. $\left.2 p_{z}\right)$. Evidently the antibonding influence of the $\operatorname{Ag}(\mathrm{II}) d^{9}$ configuration is minimal in the cationic chain; therefore, the unique, half-filled $d$ orbital is designated $d_{z}$. The crystal orbitals for the cationic chain must be dominated by the contributions from $4 d_{z}$ of $\mathrm{Ag}(\mathrm{II})$ and $2 p_{z}$ of $\mathrm{F}$. Since this signifies a half-filled conduction band, a Peierls distortion is anticipated. In this room-temperature structure, however, no such distortion is manifest. Indeed, each $\mathrm{Ag}(\mathrm{II})$ is in a highly polar electric field, since all four nearest boron atoms of each $\mathrm{Ag}(\mathrm{II})$ are either above or below it with respect to the chain direction. Nevertheless, the two intrachain Ag--F distances are remarkably close at $2.002(3)$ and 2.009 (3) $\AA$. (The difference can be readily reconciled by the way in which the anion packing dictates that the four additional anion $\mathrm{F}$ ligands directed toward each $\mathrm{Ag}(\mathrm{II})$ are not in exactly the same plane as it.)

The Peierls distortion to be expected for the $(\mathrm{AgF})_{n}^{\mathrm{n}^{+}}$chain is an alternation of linear, symmetrical $\left(\mathrm{F}-\mathrm{Ag}^{\mathrm{III}}-\mathrm{F}\right)^{+}$species and $\mathrm{Ag}^{+}$, interatomic distances in the chain becoming short about the $\mathrm{Ag}(\mathrm{III})$ and long about the $\mathrm{Ag}(\mathrm{I})$. But the polar field about each $\mathrm{Ag}(\mathrm{II})$ in the chain (see Figure 6.2) works against a centrosymmetric $\mathrm{F}$ ligand 
arrangement at each $\mathrm{Ag}$ atom. The $\mathrm{F}$ ligands of the cation do not experience such a polar field. It is perhaps for these reasons that the magnetic susceptibility data show no evidence of a Peierls transition between 6 and $280 \mathrm{~K}$.

Table 6.2. Positional Parameters for $\mathrm{AgFBF}_{4}$ at $293 \mathrm{~K}$

\begin{tabular}{cccc}
\hline & $x$ & $y$ & $z$ \\
\hline $\mathrm{Ag}$ & 0.25 & 0.25 & $0.19212(7)$ \\
$\mathrm{B}$ & 0.25 & 0.75 & 0 \\
$\mathrm{~F} 1$ & 0.25 & 0.25 & $0.6930(7)$ \\
$\mathrm{F} 2$ & $0.3136(3)$ & $0.5919(2)$ & $0.1988(5)$ \\
\hline
\end{tabular}

Table 6.3. Thermal Parameters for $\mathrm{AgFBF}_{4}$ at $293 \mathrm{~K}$

\begin{tabular}{ccccccc}
\hline & $U(1,1)$ & $U(2,2)$ & $U(3,3)$ & $U(1,2)$ & $U(1,3)$ & $U(2,3)$ \\
\hline $\mathrm{Ag}$ & $0.01496(6)$ & $\mathrm{U}(1,1)$ & $0.00654(9)$ & 0 & 0 & 0 \\
$\mathrm{~B}$ & $0.021(1)$ & $\mathrm{U}(1,1)$ & $0.017(2)$ & 0 & 0 & 0 \\
$\mathrm{~F} 1$ & $0.0341(9)$ & $\mathrm{U}(1,1)$ & $0.009(1)$ & 0 & 0 & 0 \\
$\mathrm{~F} 2$ & $0.047(1)$ & $0.0206(6)$ & $0.0315(8)$ & $0.0031(7)$ & $-0.0065(8)$ & $0.0013(7)$ \\
\hline
\end{tabular}

Table 6.4. Interatomic Distances $(\AA)$ and Angles $\left(^{\circ}\right)$ for $\mathrm{AgFBF}_{4}$

\begin{tabular}{cccccc}
\hline \multicolumn{2}{c}{ Distances } & \multicolumn{4}{c}{ Angles } \\
\hline Ag-F1 & $2.002(3)$ & F1-Ag-F1' & 180 & 2x F2-Ag-F2 & $178.68(9)$ \\
Ag-F1' & $2.009(3)$ & $4 \times$ F1-Ag-F2 & $90.66(5)$ & $4 \times$ F2-B-F2 & $109.14(8)$ \\
Ag-F2 & $2.330(2)$ & $4 \times$ F1'-Ag-F2 & $89.34(5)$ & $2 \times$ F2-B-F2 & $110.1(2)$ \\
B-F2 & $1.393(2)$ & 4x F2-Ag-F2 & $89.992(1)$ & & \\
\hline
\end{tabular}



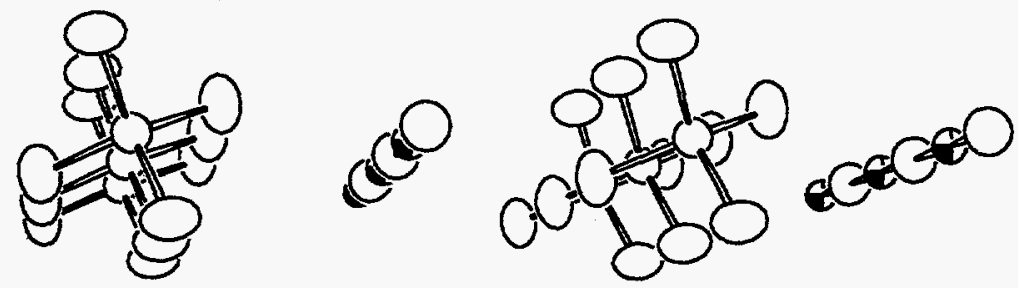

$\theta$
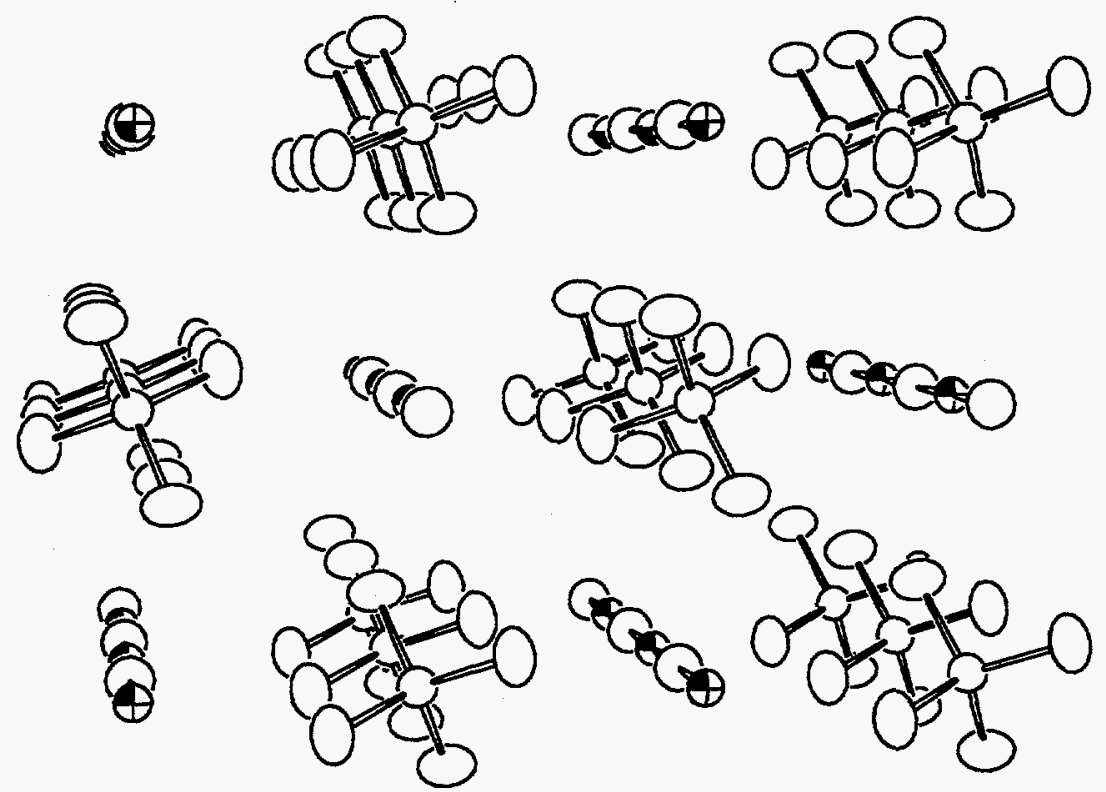

Figure 6.1. The extended structure of $\mathrm{AgFBF}_{4}$. Chains of Ag-F-Ag-F are surrounded by columns of $\mathrm{BF}_{4}^{-}$. 

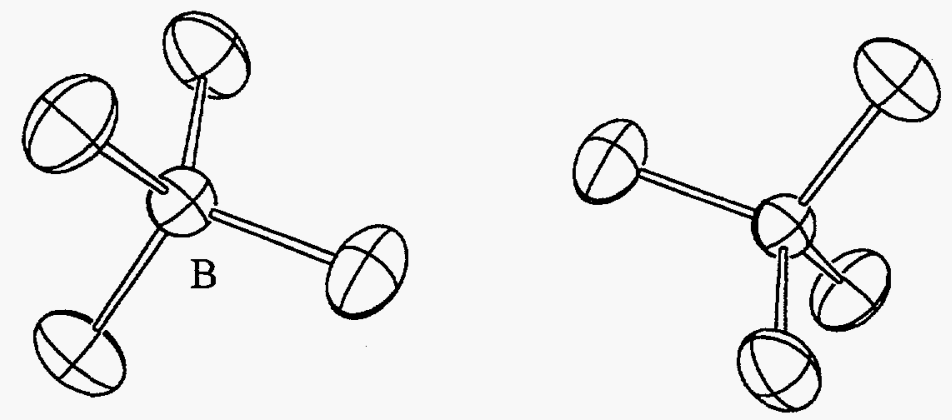

F2

F1
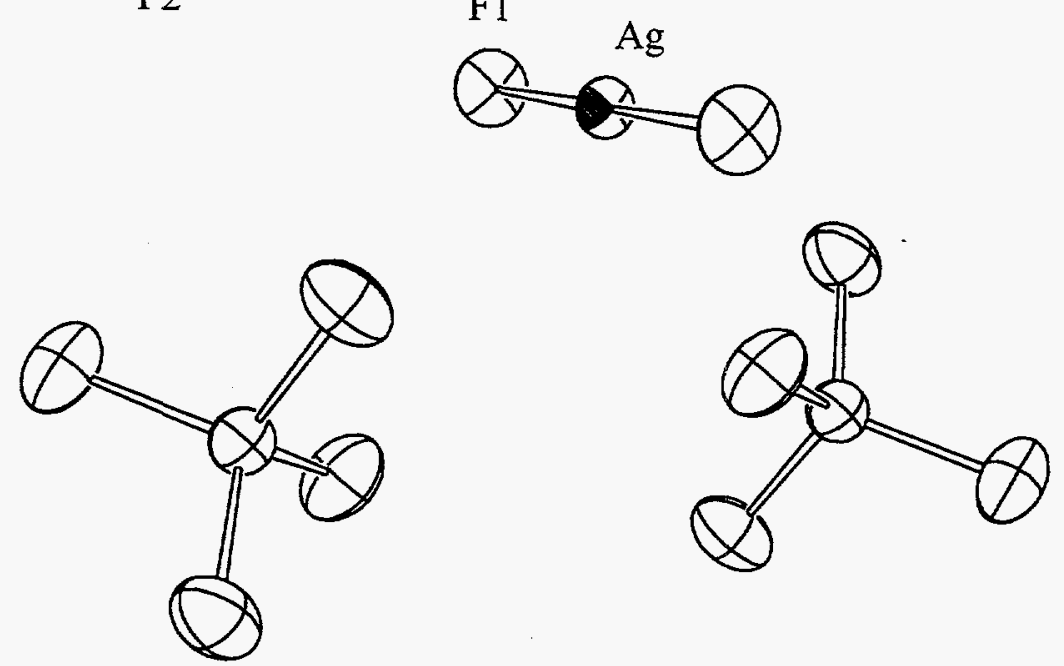

Figure 6.2. The tetragonally-distorted octahedral environment about the $\mathrm{Ag}(\mathrm{II})$ in $\mathrm{AgFBF}_{4}$.

\subsection{Structure of AgFMF $_{6}$}

\subsection{1. $M=A s, I r, A u$}

$\mathrm{X}$-ray powder diffraction data indicated that $\mathrm{AgFAuF}_{6}$ and $\mathrm{AgFIFF}_{6}$ would be isostructural with the previously determined ${ }^{1} \mathrm{AgFAsF}_{6}$. Although no attempt was made to grow crystals of the gold material, crystals of $\mathrm{AgFIrF}_{6}$ were grown from aHF solution in the same manner as that described for $\mathrm{AgFBF}_{4}$, the black material exhibiting a needle 
crystal habit. Details of the single crystal X-ray structure determination are given in Table 6.5.

Table 6.5. Crystal Data and Details of the Structure Refinement of $\mathrm{AgFIrF}_{6}$

\begin{tabular}{|c|c|}
\hline Formula, mol wt & $\mathrm{AgIrF}_{7}, 433.08$ a.u. \\
\hline Temperature & $298 \mathrm{~K}$ \\
\hline$a_{0}$ & $7.627(2) \AA$ \\
\hline$b_{0}$ & $7.068(2) \AA$ \\
\hline$c_{o}$ & $10.253(4) \AA$ \\
\hline$V$ & $552.7(3) \AA^{3}$ \\
\hline$Z$ & 4 \\
\hline Space group & P nma (No. 62) \\
\hline$d_{\text {calc }}$ & $5.23 \mathrm{~g} \cdot \mathrm{cm}^{-3}$ \\
\hline Crystal size & $0.16 \times 0.26 \times 0.23 \mathrm{~mm}$ \\
\hline Wavelength (Mo K $\alpha$ ) & $0.71073 \AA$ \\
\hline$\mu$ & $275.5 \mathrm{~cm}^{-1}$ \\
\hline Diffractometer & $\begin{array}{l}\text { Enraf-Nonius CAD4, } \\
\text { graphite monochromator }\end{array}$ \\
\hline Scan range & $3<2 \Theta<50^{\circ} ;-\mathrm{h},+\mathrm{k},-1$ \\
\hline Scan angle & $0.65^{\circ}+0.35 \tan \Theta$ \\
\hline Scan speed & $5.49 \% \mathrm{~min}$ \\
\hline Data collected & 608 \\
\hline Independent & $531 ; 451$ with $\mathrm{I}>3 \cdot \sigma(\mathrm{I})$ \\
\hline Absorption correction & $\begin{array}{l}\text { Analytical, } 10 \times 16 \times 14 \text { Gaussian Grid, } \\
\mathrm{T}_{\max }=0.079, \mathrm{~T}_{\min }=0.027\end{array}$ \\
\hline Structure solution & Based on known $\mathrm{AgFAsF}_{6}$ structure \\
\hline Refinement & $\begin{array}{l}\text { Full matrix least squares, } \\
50 \text { parameters }\end{array}$ \\
\hline Weighting scheme & $1 / \sigma^{2}$ \\
\hline Extinction correction & $F_{\text {corr }}=F_{\text {obs }} \cdot(1+g \cdot I), g=2.2 \times 10^{-7}$ \\
\hline$R, R^{\prime}$ & $0.026,0.033$ \\
\hline$w R$ & 0.030 \\
\hline Goodness-of-fit & 1.235 \\
\hline
\end{tabular}


Table 6.6. Positional Parameters for $\mathrm{AgFIrF}_{6}$ at $298 \mathrm{~K}$

\begin{tabular}{clll}
\hline & \multicolumn{1}{c}{$x$} & \multicolumn{1}{c}{$y$} & \multicolumn{1}{c}{$z$} \\
\hline $\mathrm{Ag}$ & $0.1368(1)$ & 0.25 & $0.2431(1)$ \\
$\mathrm{Ir}$ & $0.29858(8)$ & 0.25 & $0.55958(1)$ \\
$\mathrm{F} 1$ & $0.387(1)$ & 0.25 & $0.1931(9)$ \\
F2 & $0.523(1)$ & 0.25 & $0.4821(9)$ \\
F3 & $0.082(1)$ & 0.25 & $0.632(1)$ \\
F4 & $0.2256(9)$ & $0.433(1)$ & $0.4364(6)$ \\
F5 & $0.3766(9)$ & $0.063(1)$ & $0.6776(6)$ \\
\hline
\end{tabular}

Table 6.7. Thermal Parameters for $\mathrm{AgFIrF}_{6}$ at $298 \mathrm{~K}$

\begin{tabular}{ccccccc}
\hline & $U(1,1)$ & $U(2,2)$ & $U(3,3)$ & $U(1,2)$ & $U(1,3)$ & $U(2,3)$ \\
\hline Ag & $0.0103(4)$ & $0.0213(5)$ & $0.0164(5)$ & 0 & $-0.0026(4)$ & 0 \\
Ir & $0.0207(3)$ & $0.0225(3)$ & $0.0135(2)$ & 0 & $0.0004(3)$ & 0 \\
F1 & $0.019(4)$ & $0.048(6)$ & $0.028(5)$ & 0 & $-0.003(4)$ & 0 \\
F2 & $0.027(5)$ & $0.060(6)$ & $0.028(5)$ & 0 & $0.010(4)$ & 0 \\
F3 & $0.031(5)$ & $0.082(8)$ & $0.047(6)$ & 0 & $0.023(5)$ & 0 \\
F4 & $0.048(3)$ & $0.035(4)$ & $0.027(3)$ & $0.009(3)$ & $-0.015(3)$ & $0.003(3)$ \\
F5 & $0.057(4)$ & $0.029(3)$ & $0.028(3)$ & $-0.001(3)$ & $-0.007(3)$ & $0.006(3)$ \\
\hline
\end{tabular}


Table 6.8. Interatomic Distances $(\AA)$ and Angles $\left({ }^{\circ}\right)$ for $\mathrm{AgFIF}_{6}$ at $298 \mathrm{~K}$.

Roman numeral designations refer to those provided ${ }^{1}$ for $\mathrm{AgFAsF}_{6}$.

\begin{tabular}{|c|c|c|c|c|c|}
\hline \multicolumn{2}{|c|}{ Distances } & \multicolumn{4}{|c|}{ Angles } \\
\hline Ag-F1 & $1.977(9)$ & $F 1-A g-F 1^{i}$ & $176.1(4)$ & $\mathrm{Ag}-\mathrm{F} 1-\mathrm{AgV}$ & $146.0(5)$ \\
\hline $\mathrm{Ag}-\mathrm{F} 1^{\mathrm{i}}$ & $2.014(9)$ & $\mathrm{F} 1-\mathrm{Ag}-\mathrm{F} 2^{\mathrm{i}}$ & $95.6(4)$ & $\mathrm{F} 1^{\mathrm{i}}-\mathrm{Ag}-\mathrm{F} 2^{\mathrm{i}}$ & $88.4(4)$ \\
\hline $\mathrm{Ag}-\mathrm{F} 2^{\mathrm{i}}$ & $2.467(10)$ & F1-Ag-F4 & $86.7(3)$ & $F 1^{i}-A g-F 4^{i v}$ & $89.9(3)$ \\
\hline $\mathrm{Ag}-\mathrm{F} 4$ & $2.461(7)$ & F1-Ag-F5ii & $88.1(2)$ & $F 1^{i}-A g-F 5^{i i}$ & $93.0(2)$ \\
\hline $\mathrm{Ag}-\mathrm{F} 5^{i i}$ & $2.311(7)$ & $\mathrm{F} 2-\mathrm{Ag}-\mathrm{F} 4^{\mathrm{iv}}$ & $148.3(2)$ & $\mathrm{F}^{2} \mathrm{i}-\mathrm{Ag}-\mathrm{F} 5^{\mathrm{ii}}$ & $73.3(2)$ \\
\hline Ir-F2 & $1.886(9)$ & $\mathrm{F} 4-\mathrm{Ag}-\mathrm{F} 5^{\mathrm{iv}}$ & $138.4(2)$ & $\mathrm{F} 4^{\mathrm{i}} \mathrm{V}-\mathrm{Ag}-\mathrm{F} 5^{\mathrm{ii}}$ & $75.2(2)$ \\
\hline Ir-F3 & $1.812(10)$ & F4-Ag-F4 & $63.3(2)$ & $\mathrm{F}^{2}{ }^{\mathrm{iii}}-\mathrm{Ag}-\mathrm{F} 5^{\mathrm{ii}}$ & $145.8(2)$ \\
\hline Ir-F4 & $1.890(7)$ & F4-Ir-F5 & $92.4(3)$ & F4iv-Ir-F5 & $177.8(3)$ \\
\hline \multirow[t]{5}{*}{ Ir-F5 } & $1.891(7)$ & F4-Ir-F4 & $86.2(3)$ & F5-Ir-F5iv & $89.0(3)$ \\
\hline & & F2-Ir-F3 & $179.3(5)$ & F3-Ir-F4 & $90.3(3)$ \\
\hline & & $\mathrm{F} 2-\mathrm{Ir}-\mathrm{F} 4$ & $89.2(3)$ & F3-Ir-F5 & $91.4(3)$ \\
\hline & & $\mathrm{F} 2-\mathrm{Ir}-\mathrm{F} 5$ & 89.1(3) & $\mathrm{Ag}^{\mathrm{V}}-\mathrm{F} 2-\mathrm{Ir}$ & $135.5(5)$ \\
\hline & & $\mathrm{Ag}-\mathrm{F} 4-\mathrm{Ir}$ & 105.1(3) & $A g{ }{ }^{-F 5}-I r$ & $147.4(4)$ \\
\hline
\end{tabular}




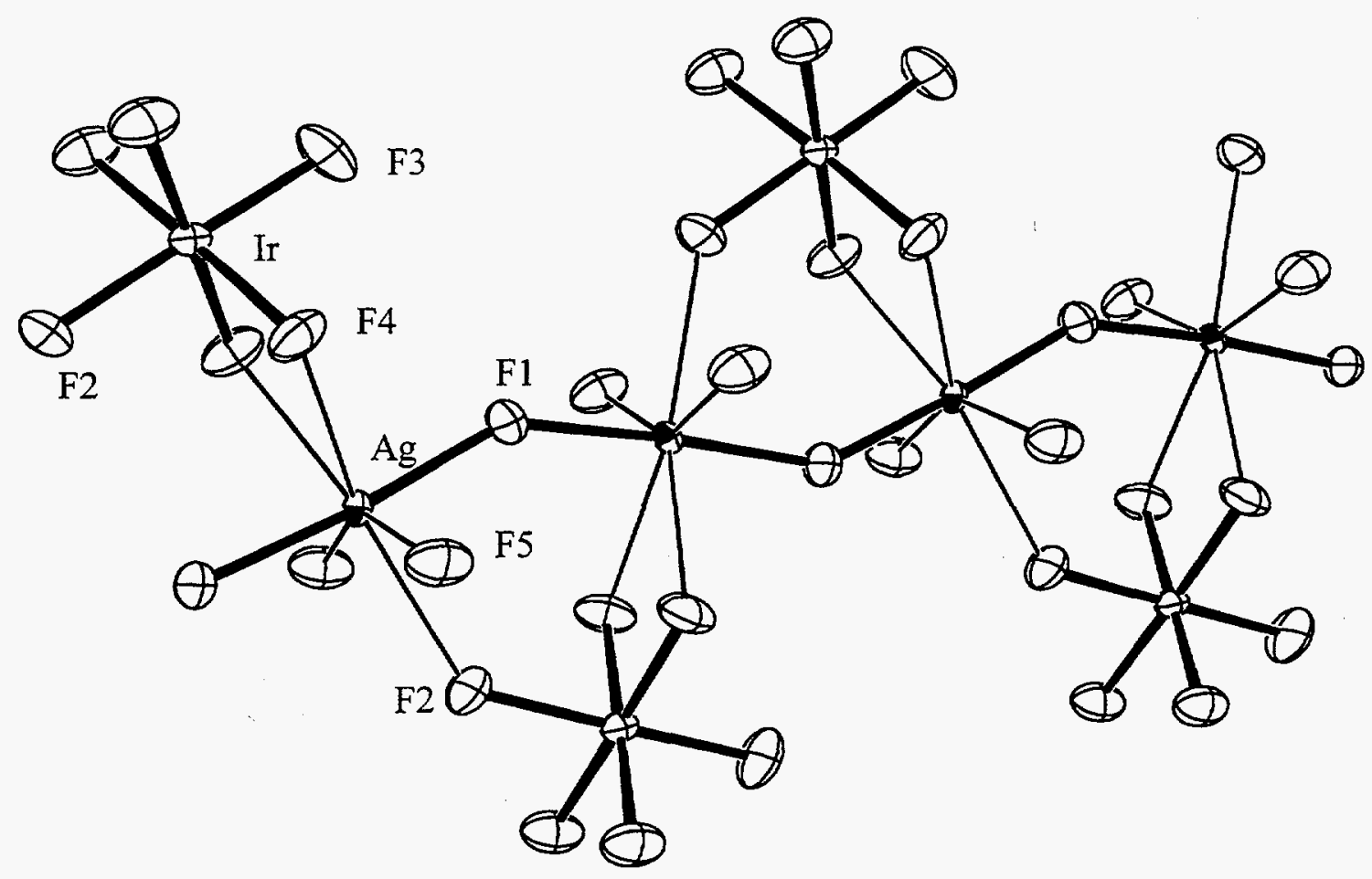

Figure 6.3. The Ag-F-Ag-F chain and its environment in $\mathrm{AgFIrF}_{6}$. 


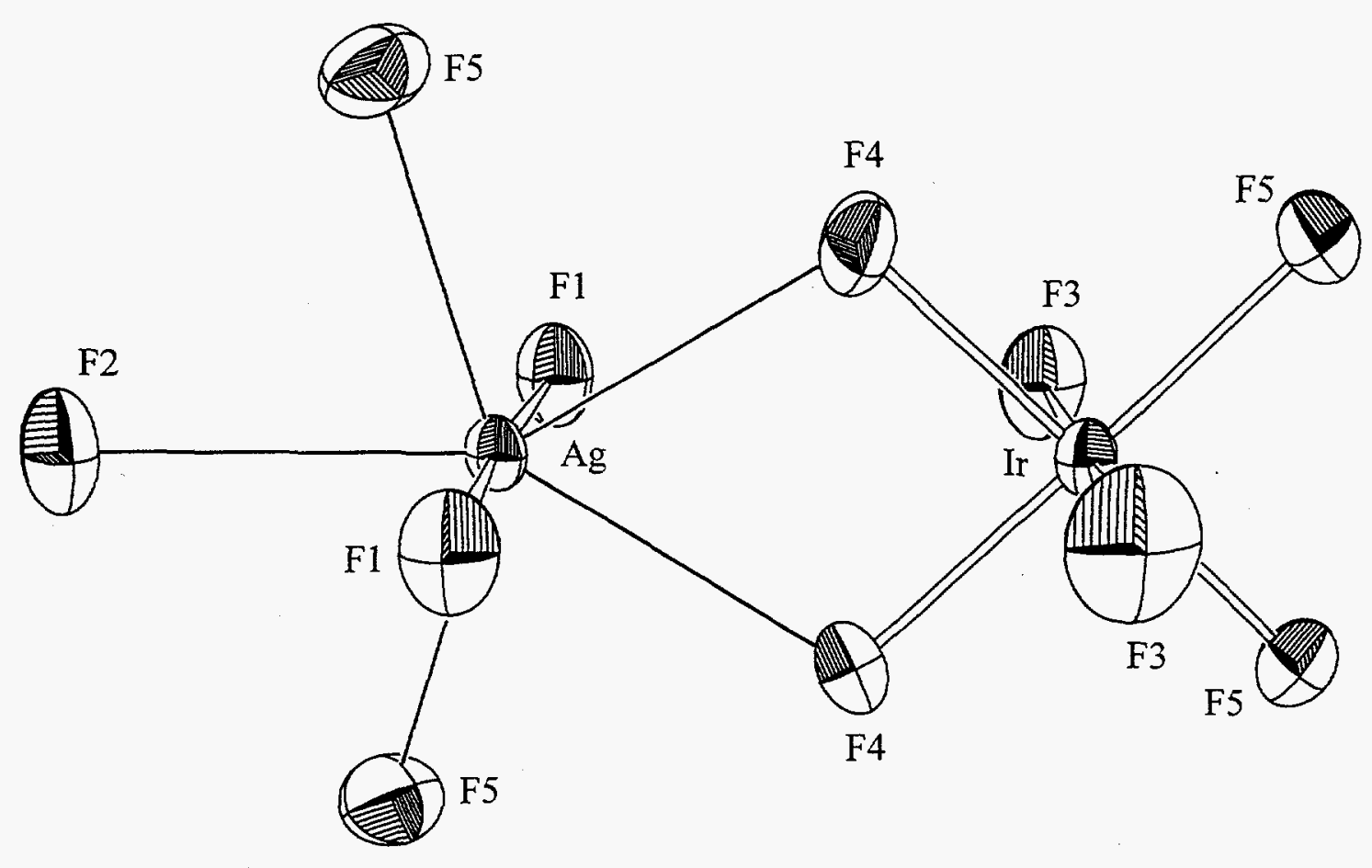

Figure 6.4. The $\mathrm{Ag}(\mathrm{II})$ and $\operatorname{Ir}(\mathrm{V})$ structural environments in $\mathrm{AgFIrF}_{6}$.

\subsection{2. $M=S b, B i$}

Several synthetic routes to $\mathrm{AgFBiF}_{6}$ have been investigated. The $\mathrm{X}$-ray powder photographs of these products show an XRPP very similar to that obtained by Casteel ${ }^{5}$ upon fluorination of $\mathrm{AgSbF}_{6}$ in an attempt to generate $\mathrm{AgFSbF}_{6}$. In the aHF, these solids appear blue and highly crystalline. However, all attempts to remove the solvent led to the crumbling of macroscopic crystals into a gray-blue powder. The XRPP of the gray-blue powder is highly complex, indicative of material whose symmetry is at least as low as 
monoclinic and possibly triclinic. No attempt to assign Miller indices to the lines of the patterns has been attempted due to their poor quality (presumably resulting from poorly crystalline material due to rapid removal of $\mathrm{HF}$ from the solvated crystals).

Consequently, very little is known of the structures of $\mathrm{AgFSbF}_{6}$ and $\mathrm{AgFBiF}_{6}$, although the magnetic data (provided in Chapter 5) indicate that polymeric $(\mathrm{AgF})_{\mathrm{n}}^{\mathrm{n}+}$ cations are present in these salts also.

\subsection{3. $M=R u$}

The X-ray powder diffraction data of $\mathrm{AgFRuF}_{6}$ exhibits a much more complicated pattern than that for the other known structures of $\mathrm{AgF}^{+}$salts. For this reason, there was great interest in obtaining a single crystal structure solution. Starting with $\mathrm{AgRuF}_{6}$ powder, crystals of $\mathrm{AgFRuF}_{6}$ were grown from aHF solution using the same technique as that used for $\mathrm{AgFBF}_{4}$. This black material exhibited a very thin rectangular plate crystal habit. Details of the structure solution are provided in Table 6.9. 
Table 6.9. Crystal Data and Details of the Structure Refinement of $\mathrm{AgFRuF}_{6}$

\begin{tabular}{|c|c|}
\hline Formula, mol wt & $\mathrm{AgRuF}_{7}, 341.93 \mathrm{amu}$ \\
\hline Temperature & $293 \mathrm{~K}$ \\
\hline$a_{o}$ & $8.3432(13) \AA$ \\
\hline$b_{o}$ & $5.4933(8) \AA$ \\
\hline$c_{o}$ & $11.9286(22) \AA$ \\
\hline$\beta$ & $108.36(1)^{\circ}$ \\
\hline$V$ & $518.9(3) \AA^{3}$ \\
\hline$Z$ & 4 \\
\hline Space group & P 2 1 /n (No. 14) \\
\hline$d_{\text {calc }}$ & $4.38 \mathrm{~g} \cdot \mathrm{cm}^{-3}$ \\
\hline Crystal size & $0.05 \times 0.13 \times 0.18 \mathrm{~mm}$ \\
\hline Wavelength (Mo K $\alpha$ ) & $0.71073 \AA$ \\
\hline$\mu$ & $66.7 \mathrm{~cm}^{-1}$ \\
\hline Diffractometer & $\begin{array}{l}\text { Enraf-Nonius CAD4, } \\
\text { graphite monochromator }\end{array}$ \\
\hline Scan range & $3<2 \Theta<60^{\circ} ; h, \pm k, \pm l$ \\
\hline Scan angle & $0.70^{\circ}+0.35 \tan \Theta$ \\
\hline Scan speed & $5.49 \% \mathrm{~min}$ \\
\hline Data collected & 3216 \\
\hline Independent & 1511,1112 with $\mathrm{I}>3 \cdot \sigma(\mathrm{I})$ \\
\hline Absorption correction & $\begin{array}{l}\text { Empirical } \Psi \text {-scans, } \\
\mathrm{T}_{\max }=1.21, \mathrm{~T}_{\min }=0.85\end{array}$ \\
\hline Structure solution & $\mathrm{Ag}$ and $\mathrm{Ru}$ atoms with Patterson map \\
\hline Refinement & $\begin{array}{l}\text { Full matrix least squares, } \\
83 \text { parameters }\end{array}$ \\
\hline Weighting scheme & $1 / \sigma^{2}$ \\
\hline Extinction correction & $F_{\text {corr }}=F_{\text {obs }} \cdot(1+g \cdot I), g=9.737 \times 10^{-7}$ \\
\hline$R, R^{\prime}$ & $0.027,0.039$ \\
\hline$w R$ & 0.028 \\
\hline Goodness-of-fit & 0.972 \\
\hline
\end{tabular}

Despite the fact that $\mathrm{RuF}_{6}^{-}$is only $\sim 0.3 \AA^{3}$ smaller ${ }^{6}$ that $\mathrm{IrF}_{6}^{-}$, the indication of the XRPP that $\mathrm{AgFRuF}_{6}$ was of a different symmetry class was confirmed by the single crystal data. As may be seen in Figure 6.6 and Table 6.12, the $\mathrm{Ag}$ (II) species in this salt is approximately square-coordinated by $4 \mathrm{~F}$ ligands. Neighboring $\mathrm{Ag}(\mathrm{II})$ are brought into 
close proximity with one another by two cis-related bridging F ligands, each nearly equidistant from two $\mathrm{Ag}(\mathrm{II})$. The nearly square $\mathrm{F}$ ligand array involves another two ligands, one from each of two $\mathrm{RuF}_{6}^{-}$. Each $\mathrm{RuF}_{6}^{-}$bridges two $\mathrm{Ag}(\mathrm{II})$, and as can be seen from Figure 6.5, all connected $\mathrm{Ag}(\mathrm{II})$ with their four near-neighbor $\mathrm{F}$ ligands lie in a common plane. The $(\mathrm{AgF})_{\mathrm{n}}^{\mathrm{n}+}$ cation is, therefore, in this case a one-dimensional ribbon.

Table 6.10. Positional Parameters for $\mathrm{AgFRuF}_{6}$ at $298 \mathrm{~K}$

\begin{tabular}{lccc}
\hline & $x$ & $y$ & $z$ \\
\hline $\mathrm{Ag}$ & $0.07248(1)$ & $0.21192(7)$ & $0.22002(1)$ \\
$\mathrm{Ru}$ & $0.22722(1)$ & $0.28784(7)$ & $-0.06154(1)$ \\
F1 & $0.0755(3)$ & $0.4622(5)$ & $-0.1900(2)$ \\
F2 & $0.1559(3)$ & $-0.0026(5)$ & $-0.1487(2)$ \\
F3 & $0.0544(3)$ & $0.2482(6)$ & $0.0034(2)$ \\
F4 & $0.3904(3)$ & $0.3208(6)$ & $-0.1335(2)$ \\
F5 & $0.2867(3)$ & $0.5730(6)$ & $0.0181(2)$ \\
F6 & $0.3696(3)$ & $0.1148(7)$ & $0.0581(2)$ \\
F7 & $0.2124(3)$ & $-0.0913(5)$ & $0.2374(2)$ \\
\hline
\end{tabular}

Table 6.11. Thermal Parameters for $\mathrm{AgFRuF}_{6}$ at $298 \mathrm{~K}$

\begin{tabular}{ccccccc}
\hline & $U(1,1)$ & $U(2,2)$ & $U(3,3)$ & $U(1,2)$ & $U(1,3)$ & $U(2,3)$ \\
\hline Ag & $0.0175(1)$ & $0.0167(1)$ & $0.0210(1)$ & $0.0004(1)$ & $0.00669(9)$ & $0.0009(1)$ \\
Ru & $0.0171(1)$ & $0.0174(1)$ & $0.0151(1)$ & $-0.0002(1)$ & $0.00541(9)$ & $-0.0004(1)$ \\
F1 & $0.028(1)$ & $0.025(1)$ & $0.021(1)$ & $0.006(1)$ & $0.0067(9)$ & $0.004(1)$ \\
F2 & $0.025(1)$ & $0.020(1)$ & $0.034(1)$ & $-0.003(1)$ & $0.0103(9)$ & $-0.009(1)$ \\
F3 & $0.030(1)$ & $0.036(1)$ & $0.028(1)$ & $-0.001(1)$ & $0.0171(8)$ & $0.000(1)$ \\
F4 & $0.0224(9)$ & $0.047(1)$ & $0.031(1)$ & $-0.002(1)$ & $0.0153(8)$ & $0.003(1)$ \\
F5 & $0.036(1)$ & $0.028(1)$ & $0.036(1)$ & $-0.008(1)$ & $0.006(1)$ & $-0.014(1)$ \\
F6 & $0.037(1)$ & $0.042(1)$ & $0.024(1)$ & $0.013(1)$ & $0.003(1)$ & $0.011(1)$ \\
F7 & $0.024(1)$ & $0.022(1)$ & $0.041(1)$ & $0.006(1)$ & $0.0085(9)$ & $0.004(1)$ \\
\hline
\end{tabular}


Table 6.12. Interatomic Distances $(\AA)$ and Angles $\left({ }^{\circ}\right)$ for $\operatorname{AgFRuF}_{6}$ at $298 \mathrm{~K}$

\begin{tabular}{lccccc}
\hline \multicolumn{2}{c}{ Distances } & \multicolumn{5}{c}{ Angles } \\
\hline Ag-F7 & $2.007(3)$ & F7-Ag-F7 & $88.64(3)$ & F1-Ru-F3 & $89.31(12)$ \\
Ag-F7 & $2.018(2)$ & F7-Ag-F1 & $176.21(11)$ & F1-Ru-F4 & $88.71(12)$ \\
Ag-F1 & $2.140(3)$ & F7-Ag-F1 & $90.82(11)$ & F1-Ru-F5 & $89.41(13)$ \\
Ag-F2 & $2.158(3)$ & F7-Ag-F2 & $90.65(11)$ & F1-Ru-F6 & $178.45(15)$ \\
Ag-F3 & $2.548(3)$ & F7-Ag-F2 & $171.68(12)$ & F2-Ru-F3 & $88.81(12)$ \\
Ag-F4 & $2.659(3)$ & F1-Ag-F2 & $89.34(11)$ & F2-Ru-F4 & $88.71(13)$ \\
Ag-F5 & $3.068(3)$ & F3-Ag-F1 & $85.29(10)$ & F2-Ru-F5 & $177.39(13)$ \\
Ru-F1 & $1.909(3)$ & F3-Ag-F2 & $83.04(10)$ & F2-Ru-F6 & $90.28(14)$ \\
Ru-F2 & $1.895(3)$ & F3-Ag-F7 & $90.95(11)$ & F3-Ru-F4 & $176.87(13)$ \\
Ru-F3 & $1.853(3)$ & F3-Ag-F7 & $88.68(11)$ & F3-Ru-F5 & $90.18(13)$ \\
Ru-F4 & $1.832(3)$ & F3-Ag-F4 & $143.93(9)$ & F3-Ru-F6 & $90.71(13)$ \\
Ru-F5 & $1.818(3)$ & F3-Ag-F5 & $158.98(8)$ & F4-Ru-F5 & $92.22(14)$ \\
Ru-F6 & $1.812(3)$ & F5-Ag-F7 & $70.39(10)$ & F4-Ru-F6 & $91.21(13)$ \\
& & F5-Ag-F7 & $81.50(10)$ & F5-Ru-F6 & $92.14(15)$ \\
& & F4-Ag-F1 & $75.39(10)$ & Ru-F1-Ag & $139.11(14)$ \\
& & F4-Ag-F2 & $66.84(10)$ & Ru-F2-Ag & $138.65(14)$ \\
& & F4-Ag-F5 & $55.15(8)$ & Ru-F3-Ag & $128.92(13)$ \\
& & Ag-F7-Ag & $155.90(16)$ & Ru-F4-Ag & $164.54(16)$ \\
& & F1-Ru-F2 & $88.17(13)$ & Ru-F5-Ag & $134.45(15)$ \\
\hline
\end{tabular}




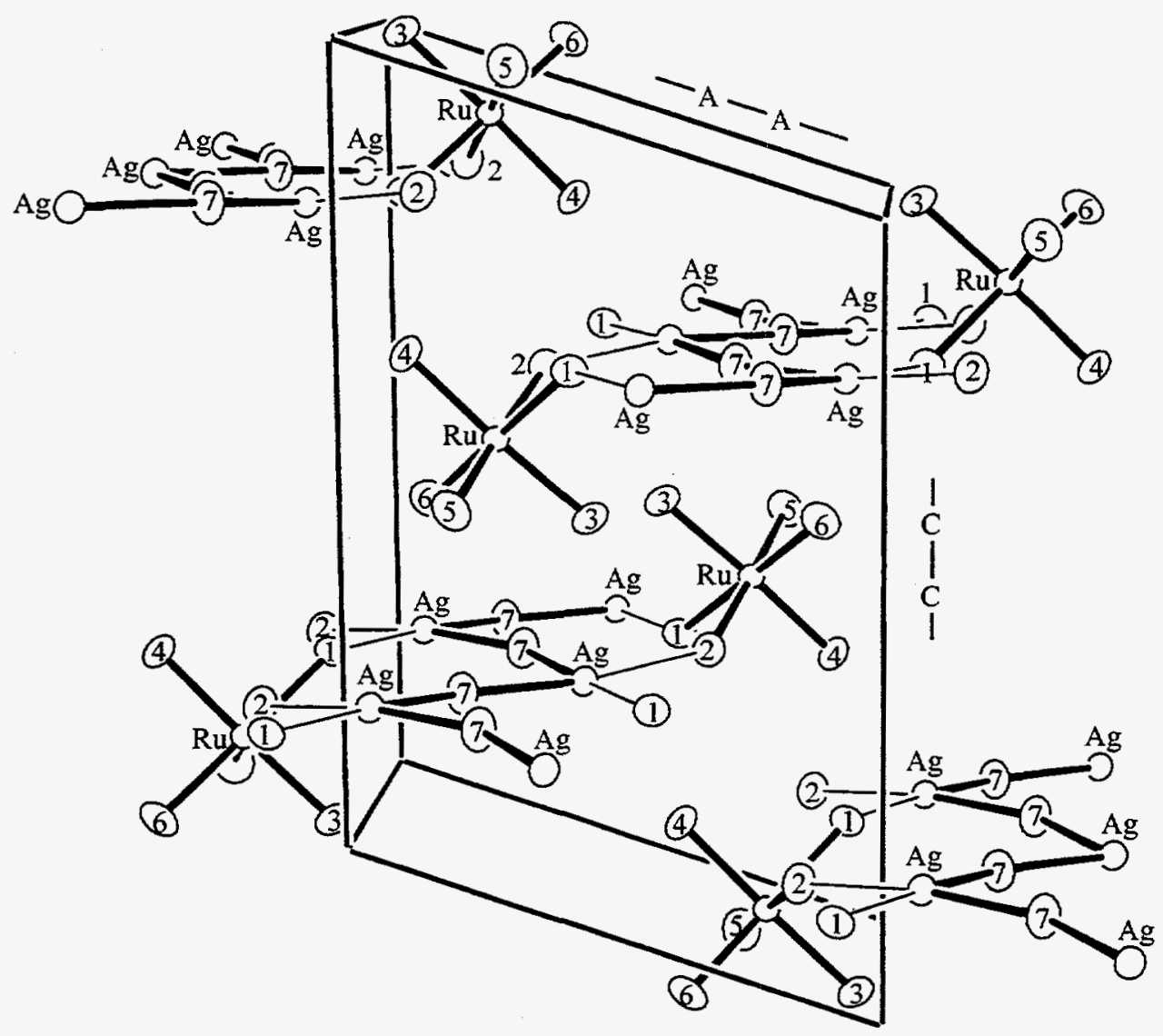

Figure 6.5. The structure of $\mathrm{AgFRuF}_{6}$, showing the one-dimensional ribbon cationic chains. 


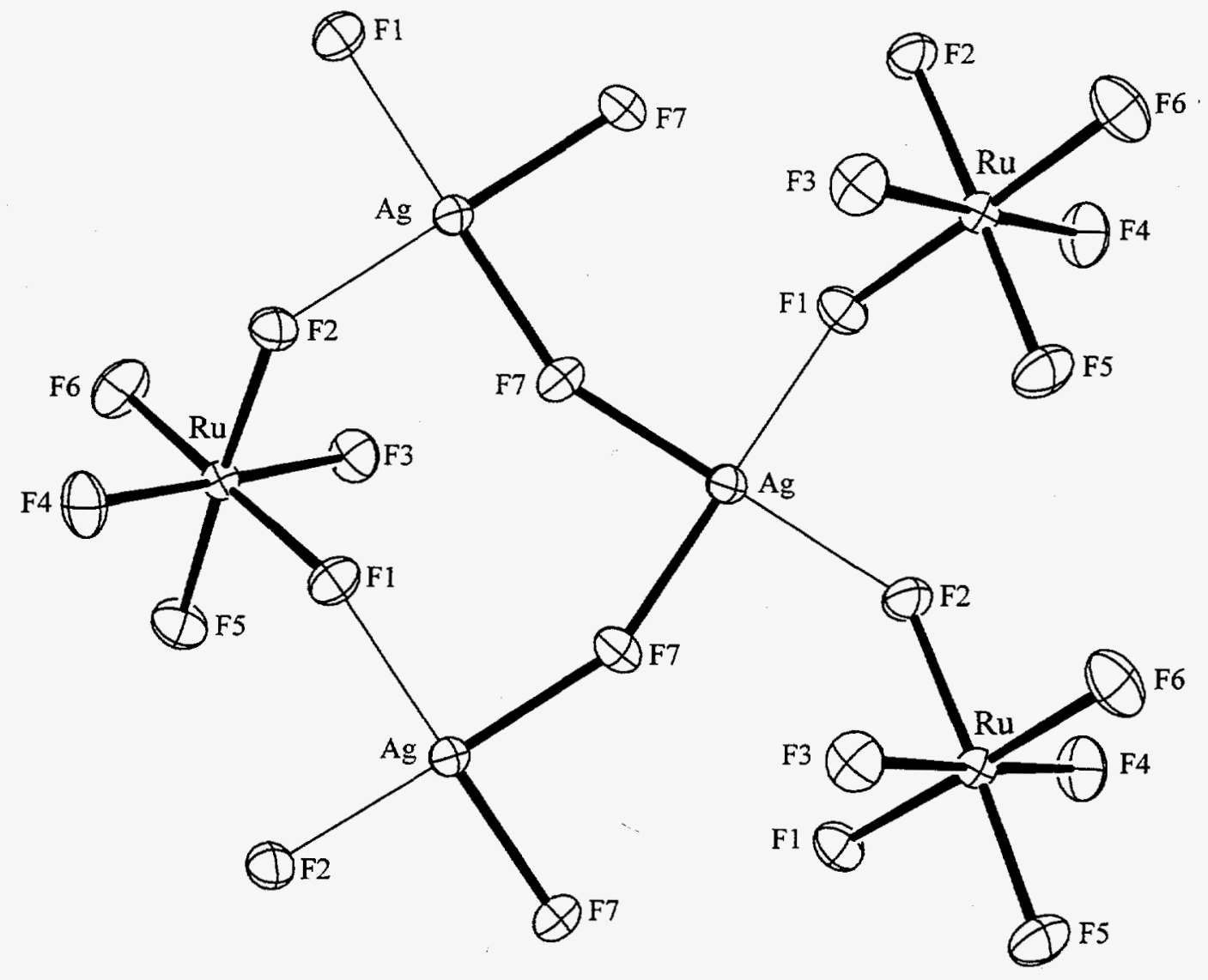

Figure 6.6. An ORTEP displaying the square-planar bonding to silver in $\mathrm{AgFRuF}_{6}$. 


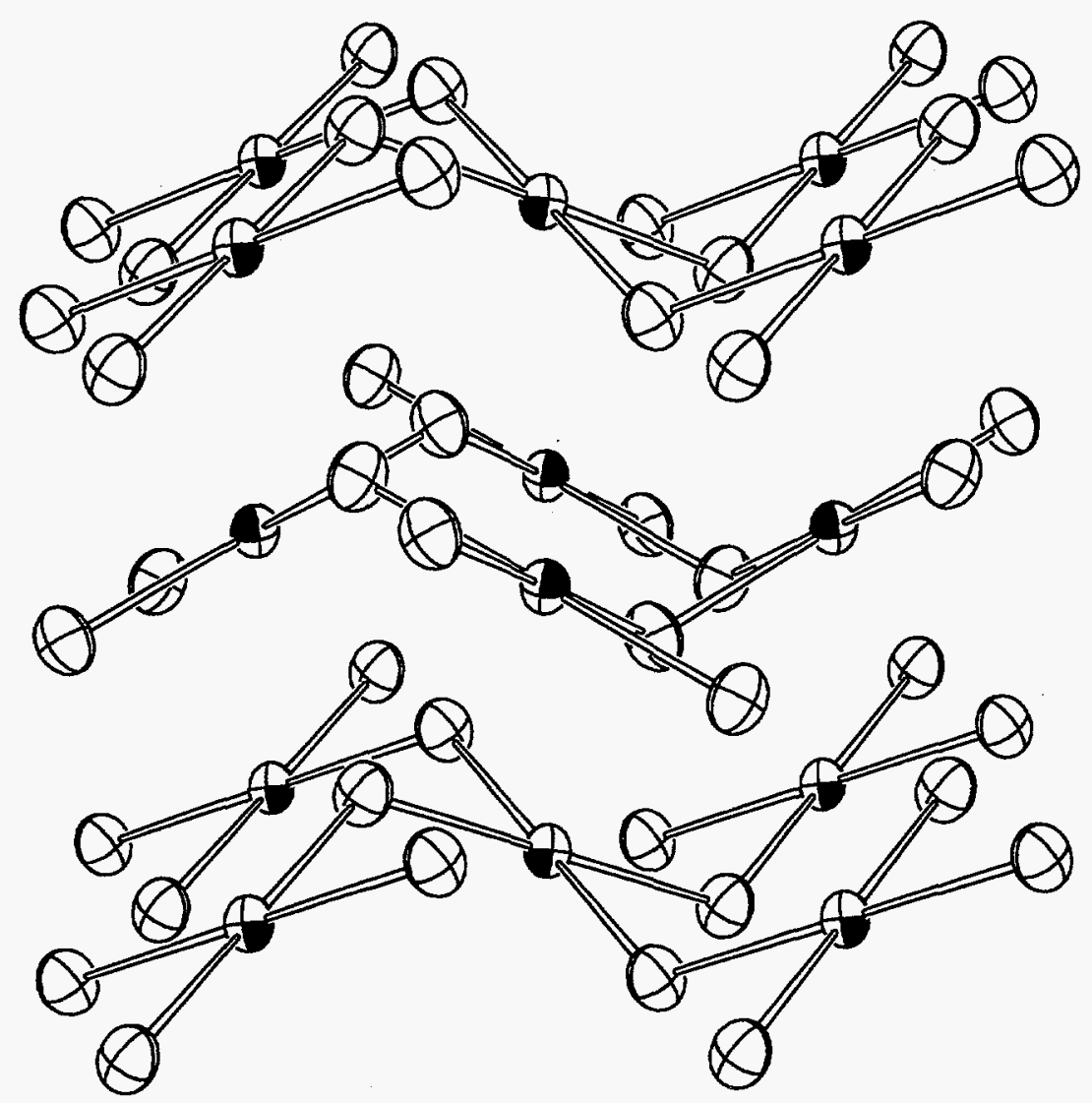

Figure 6.7. An ORTEP depicting the puckered planes of $\mathrm{AgF}_{2}$.

Figure reconstructed from data provided by Jesih et $a l^{7}$

\subsection{Optical Anisotropy in $\mathrm{AgFBF}_{4}$}

A needle of the violet $\mathrm{AgFBF}_{4}$, measuring $\sim 2.0 \mathrm{~mm}$ in length and $\sim 0.1 \mathrm{~mm}$

across, was mounted inside a carefully dried quartz capillary. The crystal was observed by transmitted light under a light microscope which was fitted with a plane polarizer in its stage. A crystal structure determination of this compound had established that its crystal habit is such that the $(\mathrm{AgF})_{\mathrm{n}}^{\mathrm{n}+}$ chain direction is coincident with the needle axis. With the axis of the needle in the plane of polarization of the light, the crystal extinguished visible 
light, appearing black through the microscope. With the plane of polarization perpendicular to the axis of the needle, the crystal transmitted the visible light, appearing violet-blue when viewed through the microscope.

\subsection{Results and Discussion}

Three general crystal symmetries have been determined to exist in the $\mathrm{AgF}^{+}$salts that are currently known, with a fourth symmetry indicated by X-ray powder diffraction data, but not yet confirmed by single-crystal studies. The highest symmetry observed is that of the tetragonal material $\mathrm{AgFBF}_{4}$. In this compound, the $\mathrm{Ag}(\mathrm{II})$ are linearly coordinated to two $\mathrm{F}$ atoms along the $(\mathrm{AgF})_{\mathrm{n}}^{\mathrm{n}+}$ chain direction, four long bonds to $\mathrm{F}$ atoms of $\mathrm{BF}_{4}^{-}$ligands completing the pseudo-octahedral environment about the silver. The $\mathrm{BF}_{4}^{-}$ anions are of such a size that packing them in a strait column provides a repeat length along $z$ that, with very little distortion of the tetrahedral ions, exactly matches the repeat length of the $(\mathrm{AgF})_{n}^{\mathrm{n}+}$ chain.

By contrast, the larger anions present in $\mathrm{AgFAsF}_{6}, \mathrm{AgFAuF}_{6}$, and $\mathrm{AgFIrF}_{6}$ are accommodated in the structure by an entirely different packing arrangement. The greater volume requirements for these anions forces the $(\mathrm{AgF})_{n}^{n+}$ chain to become kinked in order maintain the proper stoichiometry and still minimize repulsive electrostatic interactions, thus imposing a lower, orthorhombic symmetry upon the structure. Nevertheless, the kinks in the chain occur at the $\mathrm{F}$ ligand bridges while the $\mathrm{Ag}(\mathrm{II})$ still sits in a nearly linearly-coordinated $\mathrm{F}$ environment $\left\{\angle(\mathrm{F} 1-\mathrm{Ag}-\mathrm{F} 1)=176.1(4)^{\circ}\right.$ for $\left.\mathrm{AgFIrF}_{6}\right\}$ with five 
long-bonded fluorine atoms (see Figure 6.4) once again contributed by the anions, creating a compressed pseudo-pentagonal bipyramid of $\mathrm{F}$ ligands about the $\mathrm{Ag}(\mathrm{II})$.

Even though the formula unit volume of $\mathrm{AgFAsF}_{6}$ is $7.5 \AA^{3}$ less than that of its iridium relative, the structures are the same. Therefore, since $\mathrm{RuF}_{6}^{-}$is $0.3 \AA^{3}$ smaller than $\operatorname{IrF}_{6}^{-}$, it was anticipated that $\mathrm{AgF}^{+} \mathrm{RuF}_{6}^{-}$would adopt the same structure. It does not. Perhaps the most interesting feature of the monoclinic structure that is observed is that, while the $(\mathrm{AgF})_{\mathrm{n}}^{\mathrm{n}+}$ chain is still observed to exist, and the chain is bent at the $\mathrm{F}$ links $\left\{\angle(\mathrm{Ag}-\mathrm{F} 1-\mathrm{Ag})=146.0(5)^{\circ}\right.$ for $\mathrm{Ir}, \angle(\mathrm{Ag}-\mathrm{F} 1-\mathrm{Ag})=155.9(2)^{\circ}$ for $\left.\mathrm{Ru}\right\}$, we now also see a bending of the chain at the silver links. In fact, this angle $\left\{88.6(3)^{\circ}\right\}$ provides for the $\mathrm{Ag}(\mathrm{II})$ to exist in a square-coordinated $\mathrm{F}$ environment. The other two $\mathrm{F}$ ligands (each from a different anion) which lie in the plane defined by the ribbon chain are relatively short-bonded at 2.140(3) and 2.158(3) $\AA$, while the remaining two F atoms of the pseudooctahedral environment are at 2.548(3) and 2.659(3) $\AA$ (see Table 6.12 and Figure 6.5).

Such nearly square coordination of the $\mathrm{Ag}(\mathrm{II})$ is similar to that seen in the simple binary fluoride $\mathrm{AgF}_{2}$. In this compound, each $\mathrm{Ag}(\mathrm{II})$ is square-coordinated by $\mathrm{F}$ ligands at 2.086(5) $\AA$, which are equidistant from the two linked $\mathrm{Ag}(\mathrm{II})$, with $\angle(\mathrm{F}-\mathrm{Ag}-\mathrm{F})=$ 129.6(1) ${ }^{\circ}$. The resulting structure (see Figure 6.7) contains puckered sheets which can only be weakly linked to one another since the closest intersheet Ag-F distance (two per silver atom, normal to the $\mathrm{AgF}_{4}$ unit) is $2.588(6) \AA$. This intersheet distance is similar to the long-bonded contact that the $\mathrm{Ag}(\mathrm{II})$ in $\mathrm{AgFRuF}_{6}$ makes to the anions, with $\mathrm{Ag} \cdots \mathrm{F} 3=$ 
2.548(3) and $\mathrm{Ag} \cdots \mathrm{F} 4=2.659(3) \AA$. The zig-zag-Ag-F-Ag-F chain in this salt has a shorter bridging fluorine distance than in $\mathrm{AgF}_{2}\{\mathrm{Ag}-\mathrm{F} 7=2.007(3)$ and $2.018(2) \AA, \mathrm{Ag}-$ F7- $\left.\mathrm{Ag}=155.9(2)^{\circ}\right\}$, but the form of the chain is very like a section of the puckered $\mathrm{AgF}_{2}$ sheet, defined by tracing adjacent $\mathrm{Ag}(\mathrm{II})$ through cis $\mathrm{F}$ at each $\mathrm{Ag}$. The short $\mathrm{Ag}-\mathrm{F}$ distances between $\mathrm{Ag}(\mathrm{II})$ and the bridging $\operatorname{RuF}_{6}^{-}$of $\mathrm{AgFRuF}_{6}\{2.158(3)$ and $2.140(3) \AA\}$ complete the square coordination; the Ag-F distances therefore average to $2.081 \AA$, a value similar to that in $\mathrm{AgF}_{2}$. Evidently the $\mathrm{Ru}(\mathrm{V})$ centers attract $\mathrm{F}$ ligands more strongly than $\mathrm{Ag}(\mathrm{II})$. Withdrawal of 2 cis $\mathrm{F}$ ligands of the $\mathrm{AgF}_{4}$ unit, by two $\mathrm{Ru}(\mathrm{V})$, shortens the trans Ag-F linkages, which are more cationic than in $\mathrm{AgF}_{2}$ itself.

For a $d^{9}$ configuration, a Jahn-Teller distortion from octahedral symmetry results in the singly-occupied orbital being the highest in energy. In $\mathrm{AgFRuF}_{6}$, the primary distortion from octahedral symmetry about the silver atom consists of an increase in interatomic distances along the $z$-direction; in the other $\mathrm{AgF}^{+}$salts, there is a shortening of the distances in the $z$-direction. Thus, in $\mathrm{AgFRuF}_{6}$ the Jahn-Teller distortion is manifest in a lowering of the $d_{z^{2}}$ orbital energy with single occupation of the higher energy $d_{x^{2}-y^{2}}$ orbital, the occupation being reversed for the other salts of known structure.

The explanation for the four-coordination of $\mathrm{Ag}(\mathrm{II})$ in $\mathrm{AgFRuF}_{6}$ and for the twocoordination in the other known $\mathrm{AgF}^{+}$salts is uncertain. As discussed above, it does not appear likely that differing anion sizes are responsible for the appearance of two distinct geometric arrangements about the $\mathrm{Ag}(\mathrm{II})$ ion. Rather, it is more likely that the reason lies 
in the fluoro-basicity of the complexing anion. $\mathrm{RuF}_{6}^{-}$is a superior base to $\operatorname{IrF}_{6}^{-}$(i.e. $\mathrm{RuF}_{5}$ is a weaker $\mathrm{F}^{-}$acceptor than $\operatorname{IrF} \mathrm{F}_{5}$ ), so the former anion will tend to donate $\mathrm{F}^{-}$more than the latter (note the short $\mathrm{Ag}-\mathrm{F}$ interatomic distances involving $\mathrm{RuF}_{6}^{-}$ligands). Because $\mathrm{PF}_{5}$ is a relatively weak fluoro-acid and its fluoro-anion is octahedral, if $\mathrm{AgFPF}_{6}$ could ever be made, it seems likely that it would adopt the $\mathrm{AgFRuF}_{6}$ structure type. However, attempts in this laboratory by J. Münzenberg ${ }^{8}$ to synthesize $\mathrm{AgFPF}_{6}$ have failed, the reaction proceeding as depicted in Equation (1).

$$
\mathrm{AgPF}_{6} \underset{\mathrm{F}_{2}}{\stackrel{\mathrm{aHF}}{\longrightarrow}} \mathrm{AgF}_{2}+\mathrm{PF}_{5}
$$

In each of the three known structure types, there exists a one-dimensional chain cation in which adjacent $\mathrm{Ag}$ (II) centers are bridged by $\mathrm{F}$ ligands bound only to the silver atoms. The Ag-F-Ag angles vary from linear to nearly $90^{\circ}$, but the two Ag-F interatomic distances are always close to $2.0 \AA$ and not significantly different from one another. Furthermore, each of the chains is isolated from its nearest neighbor chain cations by the assembly of anions. This feature is most easily seen in $\mathrm{AgFBF}_{4}$ (see Figure 6.1), where the cation chains are linear and each one is surrounded on four sides by columns of $\mathrm{BF}_{4}^{-}$ anions. For such an $(\mathrm{AgF})_{\mathrm{n}}^{\mathrm{n}+}$ chain, the crystal orbitals will be constructed from the highest energy valence $p$ orbital of the fluorine atom in the chain $\left(p_{z}\right.$ in the case of $\mathrm{AgFBF}_{4}$ ) and from the highest energy, singly-occupied valence $d$ orbital of the $\mathrm{Ag}$ atom $\left(d_{z^{2}}\right.$ in the case of $\left.\mathrm{AgFBF}_{4}\right)$. Such a construction gives a half-filled conduction band. Magnetic susceptibility measurements have been made on many of the $\mathrm{AgF}^{+}$materials 
over a range of temperatures and fields. The data for $\mathrm{AgFBF}_{4}$ (see Chapter 5) most strikingly illustrates a trait which is shared by all of the compounds containing diamagnetic anions: an essential temperature- and field- independence over a very large temperature range. This temperature-independence is in contrast to the $1 / T$ Curie law behavior observed for localized unpaired electrons; however, it is predicted by FermiDirac statistics for partially filled bands. ${ }^{9}$ Other $\mathrm{Ag}(\mathrm{II})$ materials $\left\{\right.$ e.g. $\mathrm{Ag}\left(\mathrm{SbF}_{6}\right)_{2}$ and $\left.\mathrm{Ag}\left(\mathrm{BiF}_{6}\right)_{2}\right\}$, whose structural data indicate electronically isolated silver atoms ${ }^{10,11}$, exhibit one-electron paramagnetism (see Figure 6.8 and Figure 6.9), consistent with weakly interacting $d^{9} \mathrm{Ag}(\mathrm{II})$ cations.

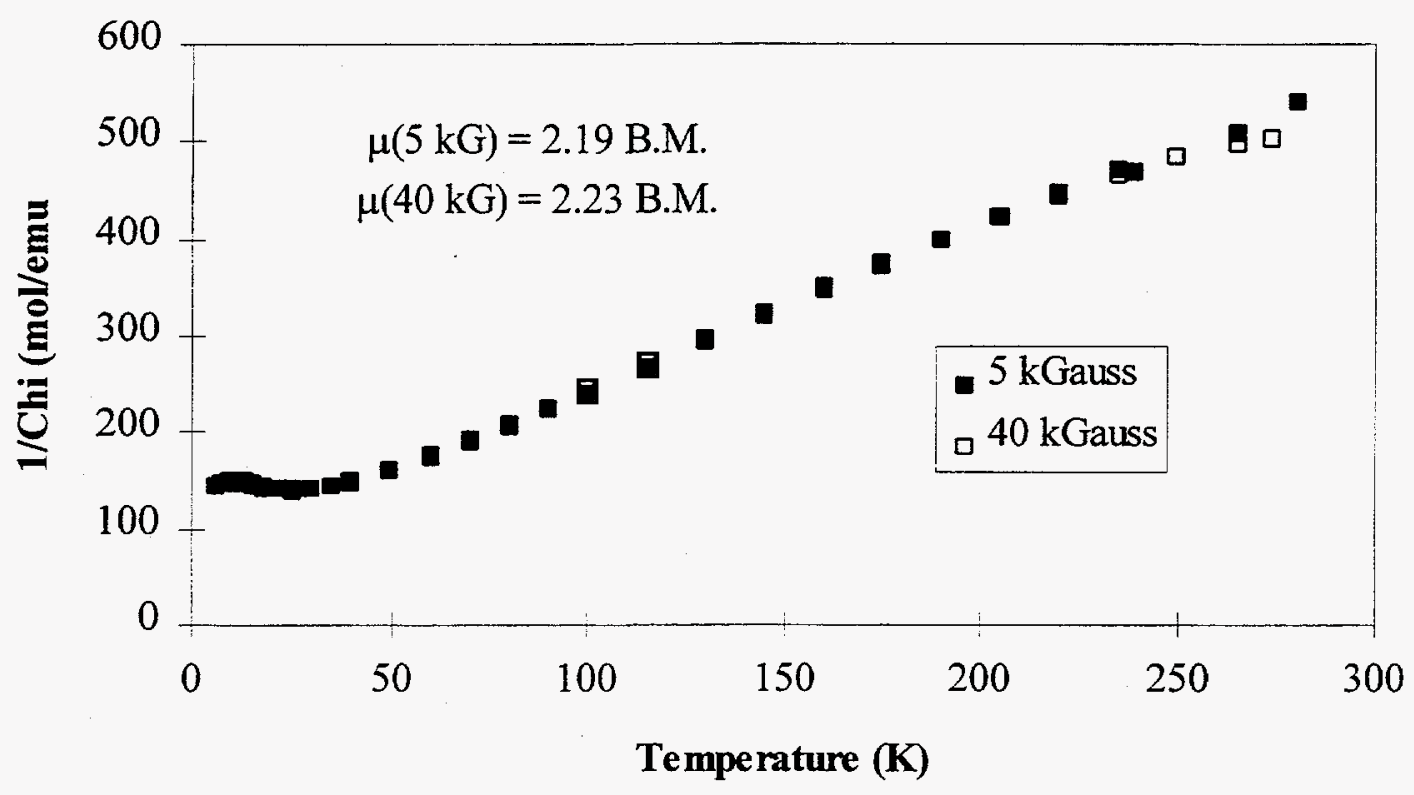

Figure 6.8. Magnetic data for $\mathrm{Ag}\left(\mathrm{BiF}_{6}\right)_{2}$. 


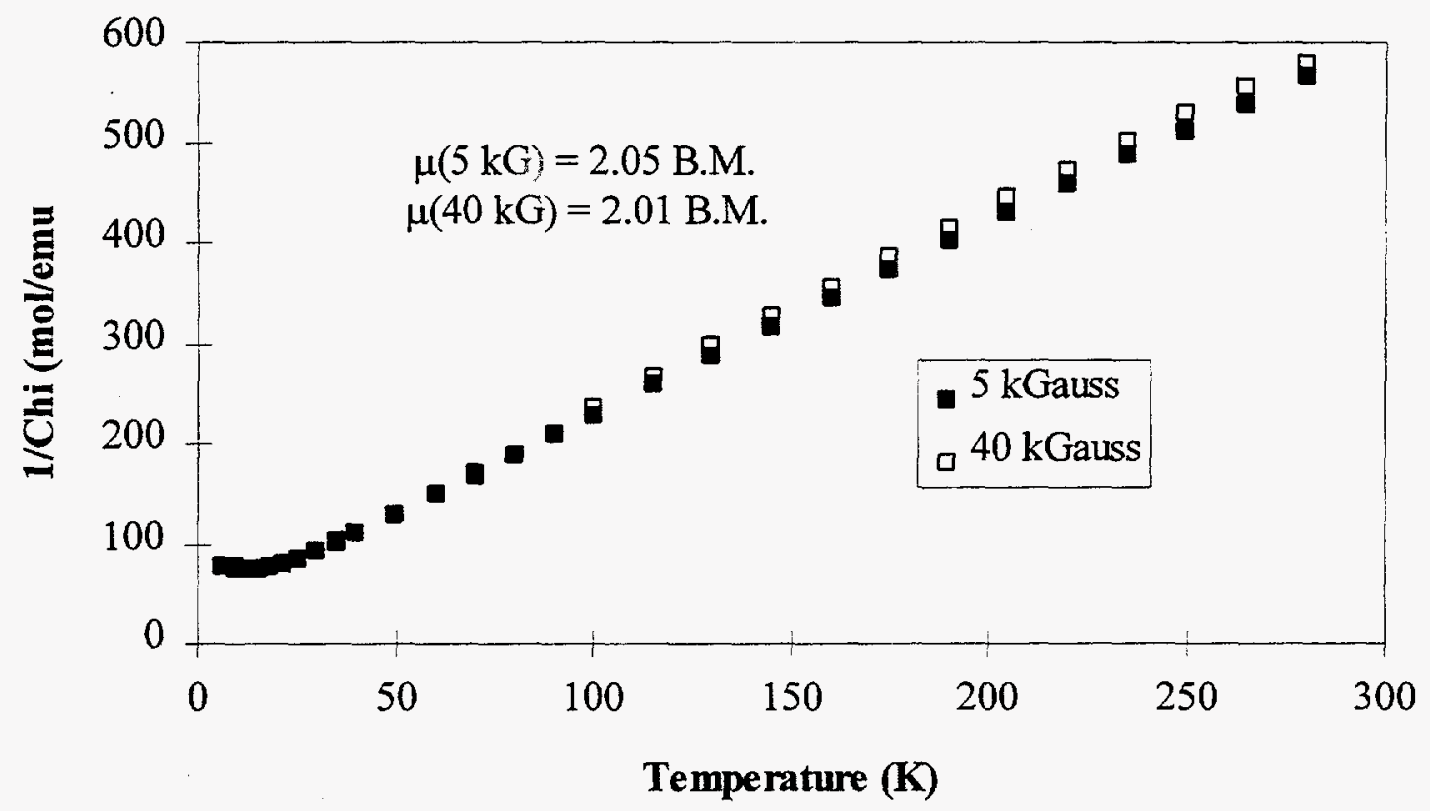

Figure 6.9. Magnetic data for $\mathrm{Ag}\left(\mathrm{SbF}_{6}\right)_{2}$.

Polarized light transmission is commonly used to identify one-dimensional electronic conductors. With the plane of polarization (i.e. the plane defined by the electric field vector and the direction of propagation vector) aligned perpendicular to the direction of conduction in a one-dimensional metal, the conduction electrons do not interact with the electro-magnetic field; the oscillating electric field is orthogonal to the direction in which the electrons can move. Consequently, the polychromatic light is both scattered and absorbed as it passes through the material and colored light may exit the 
other side of the crystal. However, with the plane of polarization aligned parallel to the direction of conduction, the oscillating electric field interacts strongly with the free electrons of the conductor, resulting in efficient reflection of visible wavelengths of light. Thus, the crystal, in this case, appears opaque. Precisely these results were obtained in a study performed with a crystal of $\mathrm{AgFBF}_{4}$. Unfortunately, attempts to directly measure a finite electrical resistance in packed powder samples of $\mathrm{AgF}^{+}$materials and along the chain direction of single crystals have all met with failure. These negative results do not preclude the existence of the delocalized electron feature, because the reactivity of the $\mathrm{AgF}^{+}$salts has prevented a conventional study of conductivity in which electrodes are painted onto the crystalline faces. However, the failure to detect a metallic conductance by this pressure contact with a gold or platinum wire does point to the possibility of these salts being sizable band gap materials, since other strong oxidizers known to be metallic are seen to be conductive in this kind of test.

Stoichiometric and structural information on the material referred to as $\mathrm{AgFBiF}_{6}$ are lacking, making a definite assignment of this formula to the material obtained impossible. Casteel synthesized an analogous material ${ }^{5}$ in a similar manner using $\mathrm{AgSbF}_{6}$ as a starting reagent. $\mathrm{X}$-ray powder diffraction data obtained by him show an XRPP almost identical to that seen for the suspected $\mathrm{AgFBiF}_{6}$. More compelling evidence for the assignment of this formula, however, is the temperature-independent magnetic susceptibility (see Chapter 5) observed for the bismuth compound, which has 
been found to be characteristic of $\mathrm{AgF}^{+}$materials. No other silver(II) system is known to produce such magnetic results.

Slow fluorination of each of $\mathrm{AgSbF}_{6}$ and $\mathrm{AgBiF}_{6}$ was found to produce lilac crystals of sufficient size for single crystal diffractometry. However, within several hours of removal of the aHF solvent, the crystals were always seen to crumble. The resulting powder gave the same poorly-crystalline, complex $\mathrm{XRPP}$ as that seen for the $\mathrm{AgFBiF}_{6}$ which was used for the acquisition of magnetic susceptibility data. It is highly probable that $\mathrm{AgFSbF}_{6}$ and $\mathrm{AgFBiF}_{6}$ crystallize with one or more solvent molecules (HF) incorporated into the lattice. When cooled to $-20^{\circ} \mathrm{C}$, the suspected $\mathrm{AgFMF}_{6} \times \mathrm{xHF}$ crystals show signs of persistence upon removal of the aHF, but the dried product then crumbles within hours under vacuum or in an inert atmosphere at room temperature. The distinctive XRPP of these salts could be a consequence of some HF involvement in the coordination of the $\operatorname{Ag}(\mathrm{II})$ species, but the magnetic susceptibility data hint that the cation is still a F-ligand bridged polymer.

\subsection{Conclusions}

Three different structure types have been unequivocally identified for the $\mathrm{AgF}^{+}$ class of materials. A fourth structure type is suggested by X-ray powder diffraction data. The three known types range in crystal symmetry from tetragonal to monoclinic, and all share the common feature of a one-dimensional, cationic $(\mathrm{AgF})_{\mathrm{n}}^{\mathrm{n}+}$ chain isolated from neighboring chains by counterions. All materials studied also exhibit temperature- 
independent magnetic susceptibility of the cation over a range from $280 \mathrm{~K}$ down to at least as low as $70 \mathrm{~K}$. These structural and magnetic features suggest the delocalization of electrons along the cationic chain, resulting in one-dimensional electronic conductivity. Polarized light transmission experiments performed on $\mathrm{AgFBF}_{4}$ support this hypothesis. Despite efforts to measure resistance in powders and single crystals, no direct evidence for such conductivity has been obtained.

$\mathrm{AgFSbF}_{6}$ and $\mathrm{AgFBiF}_{6}$ appear to be preparable and isostructural, however there is some uncertainty as to whether or not they contain aHF of crystallization. Lilac single crystals grown under aHF fell to a powder on removal of the aHF. 


\subsection{References}

${ }^{1}$ D. Gantar, B. Frlec, D. R. Russell, and J. H. Holloway, Acta Cryst., C43 (1987), pp 618-620.

${ }^{2}$ B. Žemva, R. Hagiwara, W. J. Casteel, Jr., K. Lutar, A. Jesih, and N. Bartlett, J. Am. Chem. Soc., 112 (1990), p 4846.

${ }^{3}$ W. J. Casteel, Jr., G. Lucier, R. Hagiwara, H. Borrmann, and N. Bartlett, J. Solid State Chem. 96 (1992), pp 84-96.

${ }^{4}$ B. G. Müller, Angew. Chem. Int. Ed. Engl., 26 (1987), p 688.

${ }^{5}$ W. J. Casteel, Jr., Ph. D. Thesis, (1992) Chapter 6, U.C. Berkeley.

${ }^{6} \operatorname{In} \operatorname{LiRuF}_{6}, \mathrm{R} \overline{3}, \mathrm{a}_{\mathrm{o}}=5.369(1) \AA, \alpha=56.34(1)^{\circ}, \mathrm{V} / \mathrm{Z}=100.2 \AA^{3}$, and $\operatorname{LiIrF} 6, \mathrm{R} \overline{3}, \mathrm{a}_{0}=$ $5.397(1) \AA, \alpha=55.88(1)^{\circ}, \mathrm{V} / Z=100.5 \AA^{3}$, (P. Botkovitz and G. Lucier, unpublished data) the $\mathrm{Li}^{+}$is in an octahedral hole site and the formula unit volume must closely represent the anion size in these $\mathrm{A}^{+} \mathrm{MF}_{6}^{-}$salts.

${ }^{7}$ A. Jesih, K. Lutar, B. Žemva, B. Bachmann, St. Becker, B. G. Müller, and R. Hoppe, Z. Anorg. Allg. Chem., 588 (1990), pp 77-83.

${ }^{8}$ J. Münzenberg, unpublished results, (1993), U.C. Berkeley

${ }^{9}$ P. A. Cox, The Electronic Structure and Chemistry of Solids, (1987), Oxford: Oxford University Press, pp 66-69.

${ }^{10}$ D. Gantar, I. Leban, and B. Frlec, J. Chem. Soc. Dalton Trans., (1987), pp 2379-2383. 
${ }^{11}$ G. Lucier, J. Münzenberg, W. J. Casteel, Jr., and N. Bartlett, Inorg. Chem., (1995) in press. 


\section{Chapter 7}

\section{The Oxidizing Chemistry of Solvated $\mathrm{Ag}^{\text {II }}$}

\subsection{Introduction}

Earlier work in these laboratories ${ }^{1}$ had already shown that complete dissolution of $\mathrm{AgFAsF}_{6}$ in aHF occurred when one equivalent of $\mathrm{AsF}_{5}$ was added to yield a deep blue solution:

$$
\mathrm{AgFAsF}_{6}+\mathrm{AsF}_{5} \stackrel{\mathrm{aHF}}{\longrightarrow} \mathrm{Ag}_{\text {solv }}^{\text {II }}+2 \mathrm{AsF}_{6}^{-}
$$

With the stronger fluoro-acids, $\mathrm{SbF}_{5}$ and $\mathrm{BiF}_{5}$, two equivalents of acid will bring one equivalent of $\mathrm{AgF}_{2}$ into solution, and in those cases, the $\mathrm{Ag}\left(\mathrm{MF}_{6}\right)_{2}$ salts are recovered on removal of aHF. Therefore, $\mathrm{Ag}_{\text {solv }}^{\text {II }}$ can be easily and reproducibly generated. The work described in this chapter has focused on the oxidizing power of $\mathrm{Ag}_{\text {solv }}$.

Casteel and Shen had already shown ${ }^{2}$ that $\mathrm{Ag}_{\text {solv }}^{\text {II }}$ was able, at lower temperatures $\left(<-60^{\circ} \mathrm{C}\right)$, to oxidize $\mathrm{O}_{2}$ to $\mathrm{O}_{2}^{+}$, but it remained to be demonstrated how much superior to $\mathrm{AgF}^{+}$and how much inferior to $\mathrm{Ag}_{\text {solv }}^{\text {III }}$ these oxidizing capabilities were. Again, to this 
end, the hexafluoro-anions of the third transition series (see Chapter 1) provided a convenient graded set of species to be oxidized.

\subsection{Experimental}

\subsubsection{Preparation of $\mathrm{AMF}_{6}$}

See Chapter 3 for details of the syntheses of alkali hexafluorometallate(V) salts.

\subsubsection{Interaction of $\mathrm{KIrF}_{6}$ with $\mathrm{F}_{2}$ in Acidic aHF}

One tube of an FEP T-reactor was loaded with $\mathrm{KIrF}_{6}(82 \mathrm{mg}, 0.24 \mathrm{mmol})$.

Cooling the reactor tube to $-196^{\circ} \mathrm{C}, \sim 2 \mathrm{~mL}$ aHF was condensed onto the solid, and then it was warmed to room temperature. The reactor was pressurized to $\sim 1000$ torr with $\mathrm{BF}_{3}$, whereupon no sign of reaction was visible. So the reactor was further pressurized to $\sim 1500$ torr with $F_{2}$ and continuous agitation was begun. Although the solution became slightly yellow in color, this color failed a test for volatility (it did not condense in the other tube of the reactor upon cooling in a liquid $\mathrm{N}_{2}$ bath). After 1.5 days with no evidence of $\mathrm{IrF}_{6}$ generation, the $\mathrm{KIrF}_{6}$ was recovered.

\subsubsection{Oxidation of $\mathrm{IrF}_{6}^{-}$with $\mathrm{AgFAsF}_{6}$ in acidic $\mathrm{aHF}$}

$\mathrm{KIrF}_{6}$ (234 $\mathrm{mg}, 0.679 \mathrm{mmol}$ ) was loaded into one tube of a passivated FEP Treactor, and $\mathrm{AgFAsF}_{6}(257 \mathrm{mg}, 0.813 \mathrm{mmol})$ was loaded into the other. Approximately 1 $\mathrm{mL}$ aHF was condensed onto each of the solids at $-196^{\circ} \mathrm{C}$ and then warmed to room temperature. $\mathrm{AsF}_{5}(\sim 1.3 \mathrm{mmol})$ was introduced into the reactor, resulting in the dissolution of the $\mathrm{AgFAsF}_{6}$ to give a blue solution. This was poured into the other arm of 
the reactor (where most of the $\mathrm{KIrF}_{6}$ remained as undissolved solid). There was some effervescence as a yellow precipitate was formed and the solution turned blue-green. A small aliquot of aHF was back-condensed and then poured over to flush any remaining $\mathrm{AgFAsF}_{6}$ solution from the walls of the tube; this back-distillation was repeated once more. All volatile compounds were condensed over into the clean tube of the reactor, this condensate appearing bright yellow in color. An abundance of $\mathrm{SF}_{4}$ was let into the reactor at $-196^{\circ} \mathrm{C}$ and yielded a colorless solution as the mixture melted, the surplus $\mathrm{AsF}_{5}$ combining with the $\mathrm{SF}_{4}$ in a simple acid base reaction \{see Equation (3)\} and the yellow $\mathrm{IrF}_{6}$ combining in a redox reaction as shown in Equation (4).

$$
\begin{gathered}
\mathrm{SF}_{4(\mathrm{~g})}+\mathrm{AsF}_{5(\mathrm{~g})} \stackrel{\mathrm{aHF}}{\longrightarrow} \mathrm{SF}_{3} \mathrm{AsF}_{6 \text { (solv) }} \\
3 \mathrm{SF}_{4(\mathrm{~g})}+2 \mathrm{IrF}_{6(\mathrm{~g})} \stackrel{\mathrm{aHF}}{\longrightarrow} 2 \mathrm{SF}_{3} \mathrm{IrF}_{6 \text { (solv })}+\mathrm{SF}_{6(\mathrm{~g})}
\end{gathered}
$$

An off-white powder was obtained upon removal of the volatile gases and aHF under dynamic vacuum. Because an imprecisely measured quantity of $\mathrm{AsF}_{5}$ had been introduced into the reactor, no yield could be computed by gravimetry. However, the $\mathrm{XRPP}$ of the off-white powder showed patterns belonging to both $\mathrm{SF}_{3} \mathrm{AsF}_{6}$ and $\mathrm{SF}_{3} \mathrm{IrF}_{6}$. The XRPP of the reaction byproduct showed patterns belonging to $\mathrm{AgIrF}_{6}$ and $\mathrm{KAsF}_{6}$.

\subsubsection{Oxidation of $\operatorname{IrF}_{6}^{-}$with $\mathrm{Ag}\left(\mathrm{SbF}_{6}\right)_{2}$}

One tube of an FEP T-reactor was loaded with $\operatorname{LiIrF}_{6}(104 \mathrm{mg}, 0.332 \mathrm{mmol})$ and $\mathrm{Ag}\left(\mathrm{SbF}_{6}\right)_{2}(332 \mathrm{mg}, 0.574 \mathrm{mmol})$, and aHF $(\sim 2.0 \mathrm{~mL})$ was condensed onto the mixed solids. On warming to $\sim 20^{\circ} \mathrm{C}$, the solution first became royal blue and then dark green 
as a yellow solid precipitated out of solution. The $\mathrm{aHF}$ and the volatile, yellow $\operatorname{IrF}_{6}$ were condensed into the other arm of the reactor. $\mathrm{SF}_{4}$ was introduced into the reactor and produced $\mathrm{SF}_{3} \mathrm{IrF}_{6}$ ( $\mathrm{cubic}^{3}, \mathrm{a}=5.58 \AA$ ) by way of the reaction expressed in Equation (4) as the $\operatorname{IrF}_{6} /$ aHF mixture melted, resulting in a colorless solution. Volatile compounds were evacuated and the solid product dried under dynamic vacuum for $3 \mathrm{~h}$. The yield of $\mathrm{SF}_{3} \mathrm{IrF}_{6}$ was $65.4 \mathrm{mg}(0.165 \mathrm{mmol})$, i.e. $50 \%$ of $\operatorname{Ir}$ in the $\mathrm{LiIrF}_{6}$.

\subsubsection{Interaction of $A g F I r F_{6}$ with $\mathrm{BiF}_{5}$ in the Presence of $\mathrm{F}_{2}$} $\mathrm{AgIrF}_{6}(107 \mathrm{mg}, 0.259 \mathrm{mmol})$ and $\mathrm{BiF}_{5}(315 \mathrm{mg}, 1.04 \mathrm{mmol})$ were loaded into one arm of a passivated FEP T-reactor. Approximately $2.1 \mathrm{~mL}$ aHF was condensed onto the solids at $-196^{\circ} \mathrm{C}$, and then warmed to room temperature. No reaction was evident as a clear solution above orange solid resulted. Fluorine gas was introduced into the reactor, bringing the total internal pressure to 1500 torr. Continuous agitation was maintained for $4 \mathrm{~h}$. During this time, the orange solid was slowly replaced by a mix of black and white solids (particles of each color were plainly visible) and a yellow solution. The yellow color in the HF was found to be vacuum transferable, and so was condensed, along with the $\mathrm{HF}$, into a single-tube reactor. $\mathrm{SF}_{4}$ was introduced into this reactor. As the yellow material warmed and the $\mathrm{HF}$ melted, interaction with the $\mathrm{SF}_{4}$ resulted in a colorless solution \{see Equation (4)\}. Removal of all the volatile species left a grey solid mix in the T-reactor and light yellow $\mathrm{SF}_{3} \mathrm{IrF}_{6}(87 \mathrm{mg}, 0.22 \mathrm{mmol}, 85 \%$ yield $)$ in the single-tube reactor. The XRPP of the grey solid mix showed no recognizable pattern. 


\subsubsection{Attempted Oxidation of $\mathrm{PtF}_{6}^{-}$}

$\mathrm{KPtF}_{6}(119 \mathrm{mg}, 0.342 \mathrm{mmol})$ and $\mathrm{AgFAsF}_{6}(228 \mathrm{mg}, 0.723 \mathrm{mmol})$ were loaded into separate arms of a standard FEP T-reactor. Approximately $1.5 \mathrm{~mL}$ aHF was condensed onto the $\mathrm{AgFAsF}_{6}$ and then warmed to room temperature. $\mathrm{AsF}_{5}$ was introduced into the reactor, providing for the complete dissolution of the $\mathrm{AgFAsF}_{6}$ as a royal blue solution. This solution was poured onto the dry $\mathrm{KP}_{6}$, and within seconds the solution turned green as a red-brown solid plated out on the walls of the reactor. A test for colored, volatile species was performed by condensing a small amount of HF into the other arm of the reactor. This test came up negative, so the reaction was left to agitate for $3 \mathrm{~h}$. A second test for colored, volatile species also came up negative, so the HF was decanted and removed under vacuum, leaving behind a dark blue solid.

\subsection{Results and Discussion}

$\mathrm{AgFIrF}_{6}$ is readily prepared in neutral aHF solution. However, acidification of this solution results in the liberation of $\mathrm{IrF}_{6}$. As is commonly observed with $\mathrm{AgF}^{+}$salts, acidification of the solution with a very good fluoride ion acceptor is required to dissolve the silver compound. Presumably, such conditions break down the polymeric $(\operatorname{AgF})_{n}^{n+}$ chain cation by removing fluoride from the chain and providing an abundance of solution-phase complexing anions for the $\mathrm{Ag}(\mathrm{II})$. The resulting silver species may be represented as $\mathrm{Ag}_{\text {solv }}^{\text {II }}$ \{this covers various possibilities including $\left[\mathrm{AgF}(\mathrm{HF})_{\mathrm{n}}\right]^{+}$or $\left.\left[\mathrm{Ag}(\mathrm{HF})_{\mathrm{n}}\right]^{2+}\right\}$, and, since $\mathrm{Ag}_{\text {solv }}^{\text {II }}$ had already been shown ${ }^{2}$ to oxidize $\mathrm{O}_{2}$ to $\mathrm{O}_{2}^{+}$, it was not 
surprising to find that it oxidized $\operatorname{IrF}_{6}^{-}$to $\operatorname{IrF}_{6}$. The yield of $\operatorname{IrF}_{6}$ \{as measured gravimetrically after reaction with $\mathrm{SF}_{4}$ to form $\mathrm{SF}_{3} \mathrm{IrF}_{6}$, expressed in Equation (4)\} is only about $50 \%$, the remainder of the $\operatorname{IrF}_{6}^{-}$ending up in the $\mathrm{Ag}(\mathrm{I})$ salt $\mathrm{AgIrF}_{6}$. The experimental findings indicate that $\mathrm{Ag}_{\text {solv }}^{+}$, which is generated as $\mathrm{Ag}_{\text {solv }}^{\mathrm{II}}$ oxidizes $\operatorname{IrF}_{6}^{-}$to $\mathrm{IrF}_{6}$, interacts with a second $\operatorname{IrF}_{6}^{-}$, precipitating out as the extremely low solubility salt AgIrF , thus excluding half of the $\operatorname{IrF}_{6}^{-}$from oxidation, as in Equation (5).

$$
\mathrm{Ag}_{\text {(solv) }}^{\mathrm{II}}+2 \mathrm{IrF}_{6 \text { (solv) }}^{-} \rightarrow \operatorname{IrF}_{6 \text { (g) }}+\mathrm{AgIrF}_{6 \text { (s) }}
$$

Of course, half of the $\mathrm{Ag}(\mathrm{II})$ introduced as reagent remains unreduced and eventually ends up complexed with the anion of the fluoro-acid used in the reaction. However, as was experimentally shown, in the presence of fluorine, the $\mathrm{Ag}(\mathrm{I})$ in $\mathrm{AgIrF}_{6}$ is oxidized back to $\mathrm{Ag}(\mathrm{II})$,

$$
\mathrm{AgIrF}_{6}+1 / 2 \mathrm{~F}_{2} \stackrel{\mathrm{aHF}}{\longrightarrow} \mathrm{AgFIrF}_{6}
$$

reforming $\mathrm{AgFIrF}_{6}$ which then dissolves in the acid solution, permitting more complete oxidation of the $\operatorname{IrF}_{6}^{-}$. Therefore, the addition of $F_{2}$ to the reactor is seen to greatly increase the yield (to $85 \%$ ). Because $\operatorname{IrF}_{6}$ is not generated from the interaction of $F_{2}$ with an acidified solution of $\mathrm{KIrF}_{6}$, the cationic silver is seen to behave as a catalyst in this electron oxidation of $\operatorname{IrF}_{6}^{-}$by fluorine, the overall equation being:

$$
\operatorname{IrF}_{6 \text { (solv) }}^{-}+1 / 2 \mathrm{~F}_{2} \underset{\mathrm{Ag}^{+}}{\mathrm{aHF}} \operatorname{IrF}_{6}+\mathrm{F}_{\text {(solv) }}^{-}
$$

Presumably, the addition of a catalytic amount of $\mathrm{Ag}(\mathrm{I})$ to a reactor containing $\operatorname{IrF}_{6}^{-}$, acidified aHF, and $\mathrm{F}_{2}$ can ultimately generate a quantitative yield of $\operatorname{IrF} \mathrm{F}_{6}$. 
Since $\mathrm{PtF}_{6}$ oxidizes oxygen to generate $\mathrm{O}_{2}^{+} \mathrm{PtF}_{6}^{-}$, but on sublimation dissociates to $\mathrm{O}_{2}$ and $\mathrm{PtF}_{6}$, it is evident that the oxidizing power of $\mathrm{PtF}_{6}$ is not greatly different from that of $\mathrm{Ag}_{\text {solv. }}^{\text {II }}$ Indeed, the release of $\mathrm{MF}_{6(\mathrm{~g})}$ from aHF solution provides a favorable $\mathrm{T} \Delta \mathrm{S}$ term to aid the hypothetical oxidation of the kind expressed in Equation (8).

$$
\mathrm{Ag}_{\text {(solv) }}^{\text {II }}+\mathrm{PtF}_{6 \text { (solv) }}^{-} \underset{?}{\mathrm{aHF}} \mathrm{Ag}_{(\text {(solv })}^{+}+\mathrm{PtF}_{6 \text { (g) }}
$$

Nevertheless, this oxidation did not occur. It should also be noted that it was found subsequently (see Chapter 8) that $\mathrm{PtF}_{6}^{-}$probably oxidizes $\mathrm{Ag}^{\mathrm{I}}$ to $\mathrm{Ag}^{\mathrm{II}}$ in making the pseudo-trifluoride $\mathrm{Ag}^{\mathrm{II}} \mathrm{Pt}^{\mathrm{IV}} \mathrm{F}_{6}$. Certainly $\mathrm{PtF}_{6}$ is a superior oxidizer to $\mathrm{PtF}_{6}^{\circ}$. It is therefore clear that $\mathrm{PtF}_{6}$ must be a superior oxidizer to $\mathrm{Ag}_{\text {solv. }}^{\mathrm{II}}$. Thus, it is predicted that the reverse of the reaction expressed in Equation (8) would in fact occur:

$$
\mathrm{Ag}_{\text {(solv) }}^{+}+\mathrm{PtF}_{6(\mathrm{~g})} \stackrel{\mathrm{aHF}}{\longrightarrow} \mathrm{Ag}_{\text {(solv) }}^{\mathrm{II}}+\mathrm{PtF}_{6 \text { (solv) }}^{-}
$$

this being driven by three favorable enthalpy terms: (1) the electron affinity of $\mathrm{PtF}_{6}$ $\left(\sim 184 \mathrm{kcal} \cdot \mathrm{mol}^{-1}\right),(2)$ the enhanced solvation energy of $\mathrm{Ag}_{\text {solv }}^{\text {II }}$ relative to $\mathrm{Ag}_{\text {solv }}^{+}$(because $\mathrm{Ag}^{\mathrm{II}}$ has one half-filled $d$ orbital of antibonding character whereas in $\mathrm{Ag}^{+}$that antibonding orbital is filled), and (3) the solvation energy of $\mathrm{PtF}_{6}^{-}$. The ionization enthalpy of $\mathrm{Ag}^{+}$is, of course, the adverse energy term for the reverse reaction in Equation (8). Evidently, the electron affinity of $\mathrm{Ag}_{\text {solv }}^{\text {II }}$ (i.e. the ionization enthalpy of $\mathrm{Ag}_{\text {solv }}^{+}$to give $\mathrm{Ag}_{\text {solv }}^{\text {II }}$ ) is not sufficiently high to outweigh this combination. 


\subsection{Conclusions}

Acidification of an aHF solution reproducibly generates a solution phase $\mathrm{Ag}^{\mathrm{II}}$ species, $\mathrm{Ag}_{\text {solv }}^{\mathrm{II}}$, from $\mathrm{AgF}^{+}$salts, this resulting species having a formidable electron affinity. Although $\mathrm{AgFIrF}_{6}$ is preparable, a solution of $\mathrm{Ag}_{\text {solv }}^{\mathrm{II}}$ has been experimentally shown to oxidize the $\mathrm{IrF}_{6}^{-}$anion to the neutral $\mathrm{IrF}_{6}$, thus confirming earlier findings that $\mathrm{Ag}_{\text {solv }}^{\mathrm{II}}$ is a stronger oxidizer than the polymeric cation $(\mathrm{AgF})_{\mathrm{n}}^{\mathrm{n}+}$. In fact, while fluorine gas alone does not oxidize $\mathrm{IrF}_{6}^{-}$in aHF, it does generate $\mathrm{Ag}^{\mathrm{II}}$ from $\mathrm{Ag}^{+}$, which, in acidic solution, then oxidizes the $\operatorname{IrF}_{6}^{-}$. In this interaction, the cationic silver is seen to behave as a catalyst, permitting the electron oxidation of $\mathrm{IrF}_{6}^{-}$by fluorine gas.

Consideration of the interaction of $\mathrm{Ag}_{\text {solv }}^{\mathrm{II}}$ and $\mathrm{O}_{2}$ gas, as well as that of $\mathrm{PtF}_{6}$ and $\mathrm{O}_{2}$ gas, suggests that the oxidizing potential of $\mathrm{Ag}_{\text {solv }}^{\mathrm{II}}$ is close to that of $\mathrm{PtF}_{6}$. Furthermore, entropy favors the generation of the gaseous $\mathrm{PtF}_{6}$ from the solution phase $\mathrm{PtF}_{6}^{-}$. However, in combination with the various unfavorable enthalpic terms associated with the oxidation of $\mathrm{PtF}_{6 \text { (solv) }}^{-}$, the electron affinity of $\mathrm{Ag}_{\text {solv }}^{\text {II }}$ is insufficient to affect this oxidation. Thus, $\mathrm{PtF}_{6}$ is not generated from an aHF solution of $\mathrm{Ag}(\mathrm{II})$. 


\subsection{References}

${ }^{1}$ B. Žemva, unpublished findings, (1993), U.C. Berkeley.

${ }^{2}$ W. J. Casteel, Jr., Ph. D. Thesis, (1992) Chapter 7, U.C. Berkeley, and C. Shen, Ph. D. Thesis, (1992) Chapter 8, U.C. Berkeley.

${ }^{3}$ N. K. Jha, Ph.D. Thesis, (1965) Chapter 2, U. British Columbia. 


\title{
Chapter 8
}

\author{
$\operatorname{Ag}^{\mathrm{I}} M^{\mathrm{V}} F_{6}$ vs. $\operatorname{Ag}^{\mathrm{II}} \mathbf{M}^{\mathrm{IV}} \mathbf{F}_{6}$
}

\subsection{Introduction}

Many transition metals form silver(I) hexafluorometallate(V) salts, some of which are discussed in Chapter 5. However, it was reported by Müller and Hoppe ${ }^{1}$ in 1972 that the silver hexafluorometallate salts of palladium and platinum (among others) are to be formulated as $\mathrm{Ag}^{\mathrm{II}} \mathrm{M}^{\mathrm{IV}} \mathrm{F}_{6}$. This report provided no structural information on the two materials of interest, although magnetic susceptibility data for the palladium compound was provided. The only claim to a $\operatorname{Pd}(\mathrm{V})$ complex salt is that of Falconer and his coworkers, ${ }^{2}$ who reported $\mathrm{O}_{2}^{+} \mathrm{PdF}_{6}^{-}$, but were unable to support this with any crystallographic data. The syntheses and subsequent $\mathrm{X}$-ray analyses of the two $\mathrm{AgMF}_{6}$ $(\mathrm{M}=\mathrm{Pd}, \mathrm{Pt})$ compounds in this laboratory generated, in one instance for each, an XRPP containing only a faint rhombohedral pattern. Rhombohedral symmetry is exhibited ${ }^{3}$ by the "trifluorides" of $\mathrm{Pd}$ and $\mathrm{Pt}$, which are formulated as $\mathrm{M}^{\mathrm{II}} \mathrm{M}^{\mathrm{IV}} \mathrm{F}_{6}$, as well as many of the true trifluorides, including ${ }^{4}$ those of $\mathrm{Ti}, \mathrm{V}, \mathrm{Cr}, \mathrm{Fe}, \mathrm{Co}, \mathrm{Ni}, \mathrm{Mo}, \mathrm{Ru}, \mathrm{Rh}$, and Ir. The 
divalent metals in $\mathrm{Pd}^{\mathrm{II}} \mathrm{Pd}^{\mathrm{IV}} \mathrm{F}_{6}$ and $\mathrm{Pt}^{\mathrm{II}} \mathrm{Pt}^{\mathrm{IV}} \mathrm{F}_{6}$ lie in octahedral holes as a consequence of their high-spin $d^{8}$ electron configuration. That is, the $t_{2 g}^{6} e_{g}^{2}$ configuration favors no distortion from octahedral symmetry in order to avoid spin-pairing of the two $e_{g}$ electrons. Divalent silver, however, is a $d^{9}$ species, and is predicted by Crystal Field Theory to distort to lower symmetry (Jahn-Teller distortion), thereby removing the degeneracy of the two $e_{g}$ orbitals without requiring the input of electron spin-pairing energy. A distortion seemed much less likely in a compound formulated as $\mathrm{Ag}^{\mathrm{I}} \mathrm{M}^{\mathrm{V}} \mathrm{F}_{6}$ (Crystal Field Theory predicts no distortion about a $d^{l 0} \mathrm{Ag}(\mathrm{I})$ center). Moreover, the possible existence of $\mathrm{O}_{2}^{+} \mathrm{PdF}_{6}^{-}$and the proven ability of $\mathrm{Ag}_{\text {solv }}^{\text {II }}$ to oxidize $\mathrm{O}_{2}$ to $\mathrm{O}_{2}^{+}$left open the possibility of $\operatorname{AgMF}_{6}(\mathrm{M}=\mathrm{Pd}$, $\mathrm{Pt})$ being $\mathrm{Ag}^{+} \mathrm{MF}_{6}^{-}$salts.

\subsection{Experimental}

\subsubsection{Preparation of some $A_{2} M_{6}$ salts from $A F$, metallic $M$, and $F_{2}$}

Oxidation of Pd and Pt metals by fluorine to the +4 oxidation state was accomplished at room temperature in aHF which had been made basic with alkali fluoride. This represented a new and clean synthesis of such salts. The specific details of representative reactions are provided in. Table 8.1. Because all $\mathrm{MF}_{6}^{2-}$ salts produced were very soluble in the aHF, preparations were usually performed with the metal reagent in slight excess so that pure product (identified by XRPP) could be obtained by merely decanting the supernatant solution. 
Chapter $8-A g^{I} M^{V} F_{6}$ vs. $A g^{I I} M^{I V} F_{6}$

Table 8.1. Preparation of $\mathrm{A}_{2} \mathrm{MF}_{6}$ from $\mathrm{AF}$, metallic $\mathrm{M}$, and $\mathrm{F}_{2}$

\begin{tabular}{|c|c|c|c|}
\hline $\begin{array}{c}\text { Alkali Fluoride } \\
\text { Reagent }\end{array}$ & Metal Reagent & Product and Yield & Unit Cell \\
\hline $\begin{array}{c}\mathrm{KF} \\
229 \mathrm{mg}, 3.95 \mathrm{mmol}\end{array}$ & $\mathrm{Pd}$ \\
$216 \mathrm{mg}, 2.03 \mathrm{mmol}$ & $\begin{array}{c}\mathrm{K}_{2} \mathrm{PdF}_{6} \\
529 \mathrm{mg}, 90 \%\end{array}$ & $\begin{array}{c}\text { hexagonal } \\
\mathrm{a}_{0}=5.717, \\
\mathrm{c}_{\mathrm{o}}=4.667 \AA\end{array}$ \\
\hline $\begin{array}{c}\mathrm{CsF} \\
297 \mathrm{mg}, 1.96 \mathrm{mmol}\end{array}$ & $108 \mathrm{mg}, 1.02 \mathrm{mmol}$ & $\begin{array}{c}\mathrm{Cs}_{2} \mathrm{PdF}_{6} \\
455 \mathrm{mg}, 96 \%\end{array}$ & $\begin{array}{c}\text { hexagonal }^{6}, \\
\mathrm{a}_{\mathrm{o}}=6.225, \\
\mathrm{c}_{\mathrm{o}}=5.018 \AA\end{array}$ \\
\hline $\mathrm{LiF}$ & $\mathrm{Pt}$ & $\begin{array}{c}\text { unknown, } \\
\text { poorly crystalline }\end{array}$ & \\
\hline $36 \mathrm{mg}, 1.4 \mathrm{mmol}$ & $273 \mathrm{mg}, 1.40 \mathrm{mmol}$ \\
\hline $\mathrm{KF}$ & $\mathrm{Pt}$ & $\begin{array}{c}\mathrm{K}_{2} \mathrm{PtF}_{6} \\
409 \mathrm{mg}, 100 \%\end{array}$ & $\begin{array}{c}\text { hexagonal } \\
\mathrm{a}_{\mathrm{o}}=5.778, \\
\mathrm{c}_{\mathrm{o}}=4.635 \AA\end{array}$ \\
\hline
\end{tabular}

\subsubsection{Preparation of $\mathrm{AgPtF}_{6}$}

A passivated FEP T-reactor was loaded with $\mathrm{Ag}\left(\mathrm{BiF}_{6}\right)_{2}(524 \mathrm{mg}, 0.695 \mathrm{mmol})$ in one tube and $\mathrm{K}_{2} \mathrm{PtF}_{6}(292 \mathrm{mg}, 0.753 \mathrm{mmol})$ in the other tube. After evacuating the reactor, about $2.6 \mathrm{~mL}$ aHF was condensed onto the $\mathrm{K}_{2} \mathrm{PtF}_{6}$ at $-196^{\circ} \mathrm{C}$. All of the $\mathrm{K}_{2} \mathrm{PtF}_{6}$ dissolved upon warming to room temperature, giving a yellow solution. This solution was cooled to $-60^{\circ} \mathrm{C}$, and no solid was observed to precipitate. So the solid $\mathrm{Ag}\left(\mathrm{BiF}_{6}\right)_{2}$ was slowly shaken into the solution with agitation. A brown precipitate formed rapidly. Once all of the $\mathrm{Ag}\left(\mathrm{BiF}_{6}\right)_{2}$ had been shaken into the reaction tube, two small aliquots of aHF were successively condensed over into the other tube and used to rinse any residual $\operatorname{Ag}\left(\mathrm{BiF}_{6}\right)_{2}$ over. The reaction was warmed to room temperature and left to stand for 20 min. Four successive decantations were performed, followed by back-distillation of the $\mathrm{HF}$, in order to separate the soluble reaction byproduct from the insoluble fraction. 
Following this, the reactor was pressurized to 1400 torr with $\mathrm{BF}_{3}$ and left for $15 \mathrm{~min}$. A slight blue color became apparent in the aHF, so this was carefully decanted and the $\mathrm{aHF} / \mathrm{BF}_{3}$ removed under dynamic vacuum. The products were dried for $3 \mathrm{~h}$. An X-ray powder photograph of the insoluble, brown precipitate gives a very faint rhombohedral XRPP (based on pure $\mathrm{AgPtF}_{6}$, expected: $289 \mathrm{mg}$; found: $333 \mathrm{mg}$ ). The excessive mass is perhaps due to adsorbed $\mathrm{KBiF}_{6}$ on the surface of the product, which was never exposed to liquid aHF after being brought completely to dryness and allowing the $\mathrm{KBiF}_{6}$ to crystallize properly. No XRPP was taken of the brown byproduct (based on $\mathrm{KBiF}_{6}$ product with excess $\mathrm{K}_{2} \mathrm{PtF}_{6}$, expected: $526 \mathrm{mg}$; found: $489 \mathrm{mg}$ ).

\subsubsection{Preparation of $\mathrm{AgPdF}_{6}$}

$\mathrm{Ag}\left(\mathrm{BiF}_{6}\right)_{2}(752 \mathrm{mg}, 0.998 \mathrm{mmol})$ and $\mathrm{K}_{2} \mathrm{PdF}_{6}(316 \mathrm{mg}, 1.06 \mathrm{mmol})$ were placed into separate tubes of a passivated FEP T-reactor. About $2.3 \mathrm{~mL}$ aHF were condensed onto the $\mathrm{K}_{2} \mathrm{PdF}_{6}$ at $-196{ }^{\circ} \mathrm{C}$, then warmed to room temperature. All of the $\mathrm{K}_{2} \mathrm{PdF}_{6}$ dissolved to give a yellow-orange solution. This solution was then cooled to $-60^{\circ} \mathrm{C}$, whereupon all of the material remained dissolved. The $\mathrm{Ag}\left(\mathrm{BiF}_{6}\right)_{2}$ was slowly added as a dry solid to the solution. Initially, a green solution resulted, but after just a few seconds a brown solid precipitated out and the green color faded to yellow. Once all the $\operatorname{Ag}\left(\mathrm{BiF}_{6}\right)_{2}$ had been added, the reactor was warmed to room temperature. The solution remained yellow, so the supernatant solution was decanted. Three washings of the brown solid were performed by back-distilling the HF then decanting it again. Then the reactor was 
pressurized to 1500 torr with $\mathrm{BF}_{3}$ and left for $15 \mathrm{~min}$. There was no sign of reaction to form $\mathrm{AgFBF}_{4}$ (as would have been expected had there been any $\mathrm{AgF}_{2}$ present), as was evident from the continued lack of color in the aHF column above the brown solid. So this solution was decanted and then removed under vacuum. The products were dried for $3 \mathrm{~h}$. The XRPP of the brown powder showed a material of poor crystallinity and rhombohedral pattern (like $\mathrm{Pd}^{\mathrm{II}} \mathrm{Pd}^{\mathrm{IV}} \mathrm{F}_{6}$ ). SQUID magnetometry of this product revealed it to be a simple paramagnet with a magnetic moment of 1.80 B.M. (see Figure 8.1). This value is appreciably less than the 2.0 B.M. obtained by Müller and Hoppe ${ }^{1}$ for their $\mathrm{AgPdF}_{6}$. Based on the formula $\mathrm{AgPdF}_{6}$, with the $\mathrm{Ag}(\mathrm{II})$ as the limiting component, the yield (expected: $328 \mathrm{mg}$; found: $357 \mathrm{mg}$ ) was too great to be pure (109\%). The excessive mass is perhaps due to adsorbed $\mathrm{KBiF}_{6}$ on the surface of the product, which was never exposed to liquid aHF after being brought completely to dryness and allowing the $\mathrm{KBiF}_{6}$ to crystallize properly. However, the other arm of the reactor contained $98 \%$ of the expected byproduct mass (expected: $722 \mathrm{mg} \mathrm{KBiF} 6,19 \mathrm{mg} \mathrm{K} 2 \mathrm{PdF}_{6}$; observed: $724 \mathrm{mg}$ ), which indicates a net gain in overall reactor weight (perhaps due to FEP interaction with the HF). 


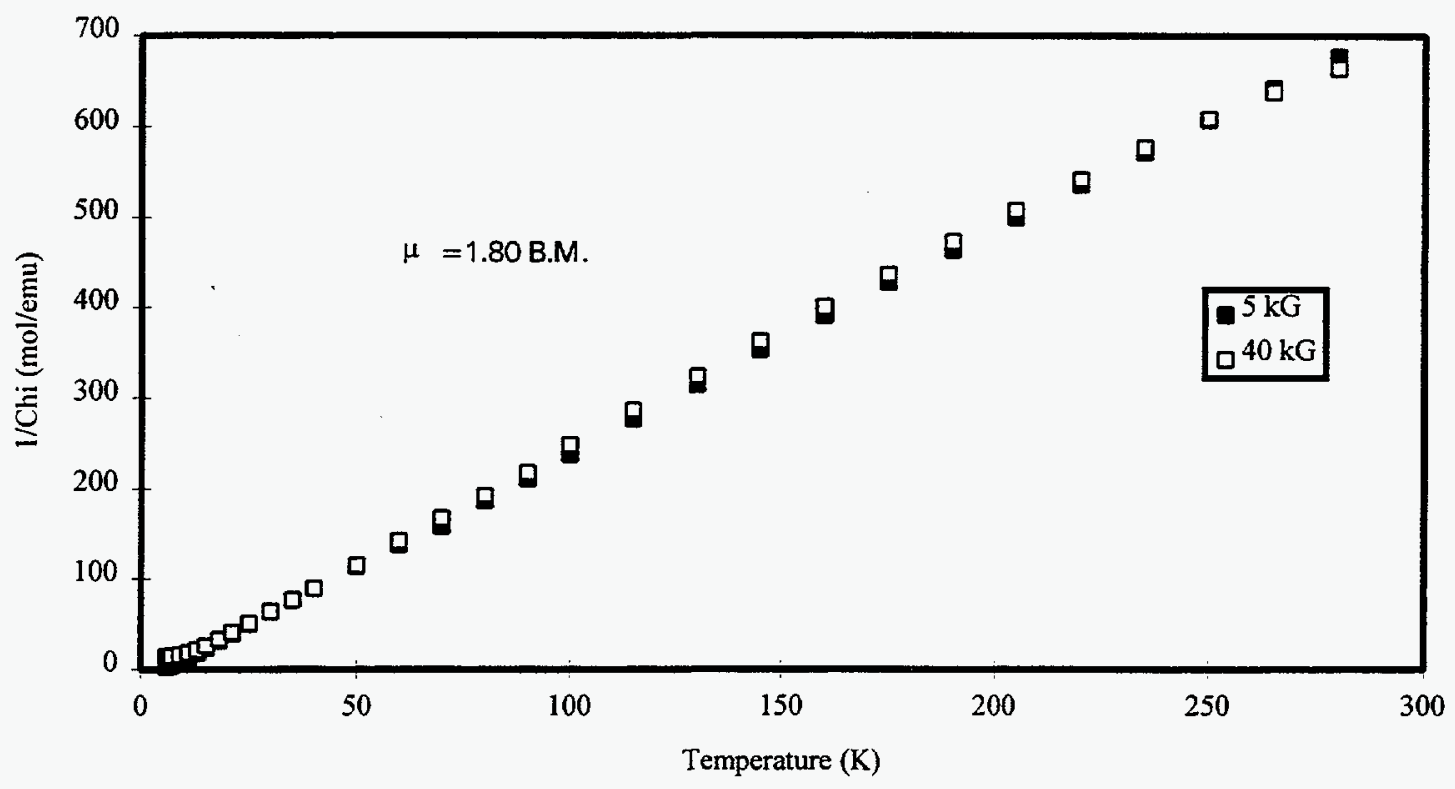

Figure 8.1. Magnetic data for $\mathrm{AgPdF}_{6}$ indicating simple paramagnetic behavior.

\subsubsection{Interaction of $\mathrm{AgF}$ with $\mathrm{KPtF}_{6}$}

A passivated FEP T-reactor was loaded with $\mathrm{KPtF}_{6}(61 \mathrm{mg}, 0.18 \mathrm{mmol})$ and $\mathrm{AgF}$ ( $43 \mathrm{mg}, 0.34 \mathrm{mmol}$ ), each reagent in a separate tube. $1.5 \mathrm{~mL}$ aHF was condensed onto the $\mathrm{AgF}$. Upon warming to room temperature, all the AgF dissolved to give a colorless solution. This was poured onto the $\mathrm{KPtF}_{6}$, immediately forming a brown precipitate. The reactor was continuously agitated for $2 \mathrm{~h}$ before decanting the colorless HF. Two washings of the brown solid were accomplished by condensing the HF back over onto it and then decanting. The HF was removed under dynamic vacuum and the product dried for $3 \mathrm{~h}$. An X-ray powder photograph of the product gives a very complicated XRPP. 
The yield (79 $\mathrm{mg}$ brown solid) was excessive for production of pure $\mathrm{AgPtF}_{6}(108 \%$ yield). And as in the previously described preparations, the excessive mass may be due to adsorbed $\mathrm{KBiF}_{6}$ on the surface of the product, which was never exposed to liquid aHF after being brought completely to dryness and allowing the adsorbed $\mathrm{K}^{+}$and $\mathrm{BiF}_{6}^{-}$to crystallize properly.

\subsubsection{Interaction of $F_{2}$ with $\mathrm{AgPtF}_{6}$ and $\mathrm{AgPdF}_{6}$}

Reactivity of $\mathrm{AgPtF}_{6}$ with $\mathrm{F}_{2}(\sim 2 \mathrm{~atm})$ in aHF was tested and resulted in no reaction. Similarly, the fluorination of $\mathrm{AgPdF}_{6}$ was attempted at $150^{\circ} \mathrm{C}$ under several atmospheres of $F_{2}$ (no aHF present) with no effect.

\subsubsection{Interaction of $\mathrm{O}_{2} \mathrm{AsF}_{6}$ with $\mathrm{PtF}_{6}^{2-}$}

$\mathrm{K}_{2} \mathrm{PtF}_{6}(87 \mathrm{mg}, 0.22 \mathrm{mmol})$ and $\mathrm{O}_{2} \mathrm{AsF}_{6}(53 \mathrm{mg}, 0.24 \mathrm{mmol})$ were loaded into separate tubes of a passivated FEP T-reactor. Approximately $1.1 \mathrm{~mL}$ aHF was condensed onto each of the reagents at $-196^{\circ} \mathrm{C}$. Upon warming the $\mathrm{HF}$ to room temperature, both solid reagents dissolved completely in their respective solutions. As the $\mathrm{O}_{2} \mathrm{AsF}_{6}$ solution was slowly poured onto the $\mathrm{K}_{2} \mathrm{PtF}_{6}$ solution, vigorous gas evolution was observed and the resulting solution became yellow-orange. After 10 min reaction time, all effervescence had ceased and no precipitate had formed. The solution was then cooled to $-196^{\circ} \mathrm{C}$ and the reactor opened to the pressure gauge. A pressure of 50 torr was measured in a total volume of approximately $85 \mathrm{~mL}$. Using the ideal gas law, a yield of $0.23 \mathrm{mmol}$ gas is calculated. The reactor was warmed to $\sim 0^{\circ} \mathrm{C}$, whereupon a yellow precipitate remained 
undissolved, so the solution was decanted. After removing the volatile species under dynamic vacuum, the products were dried for $2.5 \mathrm{~h}$. An X-ray powder photograph of the decanted orange solid gives the XRPP associated ${ }^{8,9}$ with both $\mathrm{KPtF}_{6}$ and $\mathrm{KAsF}_{6}$, but with a rhombohedral unit cell size that lies between the two (product: $a_{0}=4.94 \AA, \alpha=97.6^{\circ}$, $\mathrm{V}=117.1 \AA^{3} ; \mathrm{KPtF}_{6}: \mathrm{a}_{0}=4.96 \AA, \alpha=97.4^{\circ}, \mathrm{V}=118.7 \AA^{3} ; \mathrm{KAsF}_{6}: \mathrm{a}_{0}=4.92 \AA, \alpha=$ $97.2^{\circ}, \mathrm{V}=116.0 \AA^{3}$ ), indicating that a solid solution $\mathrm{KPtF}_{6} / \mathrm{KAsF}_{6}$ had been produced. The total mass expected $\left(\mathrm{KPtF}_{6} / \mathrm{KAsF}_{6}+\right.$ remaining $\left.\mathrm{O}_{2} \mathrm{AsF}_{6}\right)$ was $132 \mathrm{mg}$; the total mass obtained (orange decantate: $29 \mathrm{mg}$, yellow precipitate: $100 \mathrm{mg}$ ) was $129 \mathrm{mg}$.

\subsubsection{Interaction of $\mathrm{O}_{2} \mathrm{AsF}_{6}$ with $\mathrm{PdF}_{6}^{2-}$}

Similar reactivity and products were observed in reactions which used either $\mathrm{K}_{2} \mathrm{PdF}_{6}$ or $\mathrm{Cs}_{2} \mathrm{PdF}_{6}$ as the source of the palladium anion. In one of these experiments, a test for fluorine gas evolution was performed. This reaction began with $\mathrm{O}_{2} \mathrm{AsF}_{6}(142 \mathrm{mg}$, $0.643 \mathrm{mmol})$ in one tube of a passivated FEP T-reactor and $\mathrm{K}_{2} \mathrm{PdF}_{6}(193 \mathrm{mg}, 0.648$ mmol) in the other tube. Approximately $1.1 \mathrm{~mL}$ aHF was condensed onto each of the reagents at $-196^{\circ} \mathrm{C}$. Upon warming to room temperature, all the $\mathrm{K}_{2} \mathrm{PdF}_{6}$ and some of the $\mathrm{O}_{2} \mathrm{AsF}_{6}$ went into solution. The $\mathrm{O}_{2} \mathrm{AsF}_{6}$ was then cooled to $-25^{\circ} \mathrm{C}$ while the $\mathrm{K}_{2} \mathrm{PdF}_{6}$ was cooled to $0^{\circ} \mathrm{C}$. The $\mathrm{K}_{2} \mathrm{PdF}_{6}$ solution was slowly added to the $\mathrm{O}_{2} \mathrm{AsF}_{6}$, resulting in immediate effervescence and a darkening of the orange color of the solution. A light orange solid precipitated out of solution. At this point, the reaction mixture was cooled to $-78^{\circ} \mathrm{C}$ and the reactor opened to the pressure gauge. A pressure of 50 torr (equating to 
$\sim 0.2 \mathrm{mmol}$ of ideal gas at STP, although a small portion of the system was being significantly cooled) was measured. Then this gas was removed (using a static vacuum) through a column packed with $\mathrm{KF}$ followed by $\mathrm{KI}$. A small amount of discoloration of the colorless $\mathrm{KI}$ was observed, indicating the presence of $\mathrm{F}_{2}$ in the gas. After closing the reactor valve and rewarming it to room temperature, there was no further sign of reaction, so the solution was decanted. At $-196^{\circ} \mathrm{C}$, the $\mathrm{HF}$ was distilled back over but not decanted again. All volatile species were removed under dynamic vacuum and the solids dried for $2.5 \mathrm{~h}$. An XRPP of the initial orange solid showed only the pattern of pure $\mathrm{KAsF}_{6}$ (no solid solution); the solid retrieved from the highly soluble fraction gave no XRPP.

\subsubsection{Interaction of $\mathrm{K}_{2} \mathrm{PdF}_{6}$ with $\mathrm{PF}_{5}$}

$\mathrm{K}_{2} \mathrm{PdF}_{6}(133 \mathrm{mg}, 0.445 \mathrm{mmol}$ ) was placed into one tube of a passivated FEP Treactor inside the DRILAB. After evacuating the reactor on the vacuum line, about 2.0 $\mathrm{mL}$ aHF was condensed onto the orange solid at $-196^{\circ} \mathrm{C}$. Once the HF had melted and warmed to room temperature, all the $\mathrm{K}_{2} \mathrm{PdF}_{6}$ dissolved to give a light orange solution. Approximately $0.5 \mathrm{mmol} \mathrm{PF}_{5}$ was metered into the reactor, immediately giving rise to a dark solid precipitate. The reaction was intermittently cooled and then rewarmed in an attempt to ensure good dissolution of the $\mathrm{PF}_{5}$. Despite the orange color that remained in the solution, no further reaction was evident after $25 \mathrm{~min}$. The solution was decanted, the volatile components removed under dynamic vacuum, and the remaining solids (dark 
grey insoluble fraction: $68 \mathrm{mg}$; dirty yellow soluble fraction: $84 \mathrm{mg}$ ) dried overnight. Based upon the gain in total reactor weight, only $19 \mathrm{mg}=0.15 \mathrm{mmol}^{\mathrm{PF}_{5}}$ were taken up, suggesting that the reaction did not go to completion. An X-ray powder photograph of the dark grey solid gives the XRPP of $\mathrm{KPF}_{6}\left(\right.$ cubic $^{10}, \mathrm{a}_{0}=7.71 \AA$ ) with some additional, unidentified lines.

\subsubsection{Interaction of $\mathrm{K}_{2} \mathrm{PdF}_{6}$ with $\mathrm{BiF}_{5}$}

In an attempt to ensure complete reaction of a Lewis acid with the $\mathrm{PdF}_{6}^{2-}$ anion, a reaction similar to the previously described one was run, this time using a gravimetrically predetermined quantity of the strong fluoro-acid $\mathrm{BiF}_{5}$. A passivated FEP T-reactor was loaded with $\mathrm{K}_{2} \mathrm{PdF}_{6}(76 \mathrm{mg}, 0.25 \mathrm{mmol})$ in one tube and $\mathrm{BiF}_{5}(77 \mathrm{mg}, 0.25 \mathrm{mmol})$ in the other tube. After evacuation of the argon atmosphere in the reactor, $\sim 1 \mathrm{~mL}$ aHF was condensed onto each of the reagents at $-196^{\circ} \mathrm{C}$. At room temperature, all of the $\mathrm{K}_{2} \mathrm{PdF}_{6}$ dissolved to give a light orange solution, and most of the $\mathrm{BiF}_{5}$ dissolved, with a tiny speck of white solid remaining. The $\mathrm{BiF}_{5}$ solution was slowly added with agitation to the $\mathrm{K}_{2} \mathrm{PdF}_{6}$ solution. An orange precipitate formed immediately, and the reaction was intermittently agitated for $25 \mathrm{~min}$. What remained was a light orange solution above an apparently orange solid. After decanting the solution, the aHF was back-distilled onto the precipitate and decanted twice more, with a faint color remaining in the solution on the last decantation. As the aHF was removed, a flaky yellow solid $(123 \mathrm{mg})$ came out of solution; the products were vacuum dried for $3 \mathrm{~h}$. As a dry solid, the reaction precipitate 
(29 mg) had a yellow-orange appearance. An X-ray powder photograph of this material reveals a complex XRPP with moderate crystallinity.

\subsection{Results and Discussion}

The observation that $\mathrm{PtF}_{6}^{2-}$ quantitatively reduced $\mathrm{O}_{2}^{+}$to $\mathrm{O}_{2}$ in the aHF to give a 1:1 solid solution of $\mathrm{K}^{+} \mathrm{PtF}_{6}^{-}$and $\mathrm{K}^{+} \mathrm{AsF}_{6}^{-}$shows that $\mathrm{O}_{2}^{+}$is unable to coexist with $\mathrm{PtF}_{6}^{2-}$, no such salt as $\mathrm{K}^{+} \mathrm{O}_{2}^{+} \mathrm{PtF}_{6}^{2-}$ is likely to exist. But, $\mathrm{Ag}^{2+}$ was shown by Casteel and $\mathrm{Shen}^{11}$ to be approximately equivalent in oxidizing power to $\mathrm{O}_{2}^{+}$in aHF. They did so by establishing that $\mathrm{O}_{2}^{+} \mathrm{AsF}_{6}^{-}$and $\mathrm{Ag}^{+} \mathrm{AsF}_{6}^{-}$precipitate from aHF below $-60^{\circ} \mathrm{C}$, the favoring of $\mathrm{Ag}$ solv being almost complete at room temperatures:

$$
\mathrm{Ag}_{\text {solv }}^{\mathrm{II}}+\mathrm{O}_{2} \underset{\mathrm{AsF}_{5}}{\stackrel{\mathrm{aHF}}{\rightleftharpoons}} \mathrm{O}_{2}^{+} \mathrm{AsF}_{6}^{-}+\mathrm{Ag}^{+} \mathrm{AsF}_{6}^{-}
$$

On the basis of these results, the formulation of $\mathrm{AgPtF}_{6}$ as $\mathrm{Ag}^{+} \mathrm{PtF}_{6}^{-}$seems more favorable than $\mathrm{Ag}^{2+} \mathrm{PtF}_{6}^{2-}$. However, for a formulation $\mathrm{A}^{2+} \mathrm{X}^{2-}$, there is a fourfold advantage to the lattice energy compared with $\mathrm{A}^{+} \mathrm{X}$, and this could prefer the former formulation in the crystal lattice. The lattice energy for a salt $\mathrm{A}^{{ }^{+}} \mathrm{A}^{{ }^{+}} \mathrm{X}^{2-}$ is, for similarly sized cations, only about three-fourths ${ }^{12}$ that for $\mathrm{A}^{2+} \mathrm{X}^{2-}$.

Unfortunately, the structural information provided by the experimental work on $\mathrm{AgPtF}_{6}$ does not provide a definitive answer to the question is it $\mathrm{Ag}^{+} \mathrm{Pt}^{\mathrm{V}} \mathrm{F}_{6}^{-}$or $\mathrm{Ag}^{2+} \mathrm{Pt}^{\mathrm{IV}} \mathrm{F}_{6}^{2-}$. In the experiments in which $\mathrm{AgPtF}_{6}$ was precipitated from aHF by mixing $\mathrm{Ag}^{2+}\left(\mathrm{BiF}_{6}\right)_{2}$ with $\mathrm{K}_{2} \mathrm{PtF}_{6}$, the solid gave a weak $\mathrm{X}$-ray powder pattern indicative of a rhombohedral 
unit cell not very different from that of $\mathrm{Pd}^{\mathrm{II}} \mathrm{Pd}^{\mathrm{IV}} \mathrm{F}_{6}$ or $\mathrm{IrF}_{3}$. This is compatible with $\operatorname{Ag}^{\mathrm{I}} \mathrm{Pt}^{\mathrm{V}} \mathrm{F}_{6}$, but not compelling for that formulation since $\operatorname{AgIrF}_{6}$ is tetragonal ${ }^{10}\left(\mathrm{a}_{0}=4.85\right.$, $c_{0}=9.70 \AA$ ) and the platinum analogue should be the same. In experiments where $\mathrm{AgF}$ (dissolved in aHF) and $\mathrm{KPtF}_{6}$ (in aHF) were mixed to precipitate $\mathrm{AgPtF}_{6}$, the X-ray powder patterns were reproducible from one preparation to another, but complex. These complex patterns were similar in some respects to those of $\mathrm{AgF}_{3}$ and $\mathrm{AuF}_{3}$, only more complicated than in these hexagonal trifluorides. The patterns showed no $\mathrm{AgF}_{2}$ impurity nor any $\mathrm{AgIrF}_{6}$-type pattern. It is plausible to associate this pattern with the lower symmetry to be expected as a consequence of the so-called Jahn-Teller distortion of a $d^{9}$ $\mathrm{Ag}(\mathrm{II})$ species.

Magnetic susceptibility data are not available for $\mathrm{AgPtF}_{6}$, but, as in the case of $\mathrm{AgPdF}_{6}$, such data are unlikely to be helpful in resolving the formulation since $d^{9} \mathrm{Ag}(\mathrm{II})$ and $d^{5} \mathrm{Pt}(\mathrm{V})$ each have one unpaired electron with no strong coupling between the magnetic centers.

A chemical point in favor of the formulation $\mathrm{Ag}^{\mathrm{II}} \mathrm{Pt}^{\mathrm{IV}} \mathrm{F}_{6}$ is the failure to fluorinate the material in the manner of $\operatorname{AgIrF}_{6}$ (see Chapter 5), generating $\operatorname{AgFIrF}_{6}$ with $F_{2}$. It seems likely that $\mathrm{Ag}^{+} \mathrm{PtF}_{6}^{-}$would have behaved similarly.

Like the $\mathrm{AgPtF}_{6}$ precipitated from the mixing of $\mathrm{Ag}^{2+}\left(\mathrm{BiF}_{6}^{-}\right)_{2}$ and $\mathrm{K}_{2} \mathrm{PtF}_{6}$, the $\mathrm{AgPdF}_{6}$ obtained similarly using $\mathrm{K}_{2} \mathrm{PdF}_{6}$ was rhombohedral and the $\mathrm{X}$-ray powder pattern similar to that of $\mathrm{Pd}^{\mathrm{II}} \mathrm{Pd}^{\mathrm{IV}} \mathrm{F}_{6}$. The magnetic susceptibility is that of a simple 
paramagnet and consistent with either $\mathrm{Ag}^{\mathrm{I}} \mathrm{Pd}^{\mathrm{V}} \mathrm{F}_{6}$ or $\mathrm{Ag}^{\mathrm{II}} \mathrm{Pd}^{\mathrm{IV}} \mathrm{F}_{6}$. Although the latter formulation should manifest a Jahn-Teller distortion, this could be frustrated as in the platinum counterpart by the presence of a regularly octahedral $t_{2 g}^{6}$ configuration anion. It could be that slow crystallite formation in the $\mathrm{AgPtF}_{6}$ preparation from $\mathrm{AgF}$ and $\mathrm{KPtF}_{6}$ produced the lower symmetry material seen there. Since $\mathrm{KPdF}_{6}$ is as yet unknown, the counterpart of the synthesis of $\mathrm{AgPtF}_{6}$ from $\mathrm{AgF}$ and $\mathrm{KPtF}_{6}$ could not be carried out.

The successful oxidation of $\mathrm{PtF}_{6}^{2-}$ by $\mathrm{O}_{2}^{+}$in aHF raised the possibility that a similar oxidation of $\mathrm{PdF}_{6}^{2-}$ could be carried out. Persuasive evidence for the existence of $\mathrm{O}_{2} \mathrm{PdF}_{6}^{-}$ was provided by Falconer $e t a l^{2}$ in the form of Raman vibrational and magnetic susceptibility data. Although this report was not supported by crystallographic information, it did appear to be likely that the synthetic conditions were such as to favor the formation of $\mathrm{O}_{2} \mathrm{~F}$ (an excellent fluoro-base) and $\mathrm{PdF}_{5}$, so begetting $\mathrm{O}_{2}^{+} \mathrm{PdF}_{6}^{-}$.

The interaction of $\mathrm{O}_{2} \mathrm{AsF}_{6}$ with $\mathrm{K}_{2} \mathrm{PdF}_{6}$ in aHF was also carried out in an attempt to generate $\mathrm{KPdF}_{6}$. The products of this reaction were never fully characterized because of their amorphous nature. However, it was clear that the palladium ended up in two distinct phases: an insoluble orange powder, and a highly soluble red phase. The identity of the orange powder was never unambiguously determined. The XRPP of the colorless $\mathrm{KAsF}_{6}$ salt (an obvious byproduct of the reaction) dominates the $\mathrm{X}$-ray powder photograph (out to very high angle) so that no other lines are visible; and it is suspected that the color may arise from the presence of $\mathrm{KPdF}_{5} . \mathrm{PdF}_{5}^{-}$is perhaps a chain polymer in 
which each $\mathrm{PdF}_{6}$ is joined to two like neighbors by $\mathrm{F}$ bridges. If so, it could be difficult to obtain $\mathrm{KPdF}_{5}$ as a crystalline phase. Reacting $\mathrm{K}_{2} \mathrm{PdF}_{6}$ with an equimolar quantity of $\mathrm{BiF}_{5}$ was performed as a direct route to $\mathrm{KPdF}_{5}$. No oxidation reaction was possible here since $\mathrm{Bi}(\mathrm{V})$ is surely far too weak to affect the oxidation of $\mathrm{Pd}(\mathrm{IV})$. Although the poorly crystalline, and very complex, XRPP obtained from the sparingly soluble product of this reaction revealed little about the material itself, the solid did have essentially the same color as the insoluble product observed in the interaction of $\mathrm{O}_{2} \mathrm{AsF}_{6}$ with $\mathrm{K}_{2} \mathrm{PdF}_{6}$. Furthermore, the presence of $\mathrm{F}_{2}$ was detected in the evolved gas in the $\mathrm{O}_{2}^{+}$reaction, perhaps arising as a decomposition product of $\mathrm{O}_{2} \mathrm{~F}$. Thus, it seems likely that this orange material is indeed $\mathrm{KPdF}_{5}$, and that the reaction proceeded, at least in part, as follows:

$$
\mathrm{O}_{2} \mathrm{AsF}_{6 \text { (solv) }}+\mathrm{K}_{2} \mathrm{PdF}_{6 \text { (solv) }} \stackrel{\mathrm{aHF}}{\longrightarrow} \mathrm{KAsF}_{6(\mathrm{~s})}+\mathrm{KPdF}_{5(\mathrm{~s})}+\mathrm{O}_{2} \mathrm{~F}_{(\mathrm{g})}
$$

Due to the extreme solubility of the red product generated in the interaction of $\mathrm{O}_{2} \mathrm{AsF}_{6}$ and $\mathrm{K}_{2} \mathrm{PdF}_{6}$, it is most likely that this material does not contain the $\mathrm{PdF}_{6}^{-}$anion. Such solubility is generally characteristic of doubly-negative ions. So it seems much more likely that the product is either the (bis)dioxygenyl salt $\left(\mathrm{O}_{2}^{+}\right)_{2} \mathrm{PdF}_{6}^{2-}$ or perhaps $\mathrm{K}^{+} \mathrm{O}_{2}^{+}$ $\mathrm{PdF}_{6}^{2-}$. Either of these products would indicate that there exists a metathetical reaction in competition with the precipitation of $\mathrm{KPdF}_{5}$. Perhaps the $\mathrm{O}_{2 \text { (solv) }}^{+}$reacts to form $\mathrm{O}_{2} \mathrm{~F}$, as in Equation (2), until the basicity of the solution becomes too low. At this point, the $\mathrm{PdF}_{5}^{-}$ and $\mathrm{O}_{2}^{+}$compete equally for available $\mathrm{F}^{-}$ions and a stable solution containing $\mathrm{O}_{2}^{+}$and $\mathrm{PdF}_{6}^{2-}$ exists. 


\subsection{Conclusions}

Preparations of $\mathrm{AgMF}_{6}(\mathrm{M}=\mathrm{Pd}, \mathrm{Pt})$ have been investigated in an attempt to determine the oxidation states of the metal atoms in these salts. A definitive X-ray powder pattern has not been obtained in either case. The rhombohedral pattern obtained from the interaction of $\mathrm{MF}_{6}^{2-}$ with $\mathrm{Ag}(\mathrm{II})$ in aHF could be compatible with the formulation $\mathrm{Ag}^{\mathrm{I}} \mathrm{M}^{\mathrm{V}} \mathrm{F}_{6}$ and is in agreement with expectations based upon the observed oxidation of $\mathrm{PtF}_{6}^{2-}$ by $\mathrm{O}_{2}^{+}$. However, the favorable lattice energy of $\mathrm{Ag}^{2+} \mathrm{MF}_{6}^{2-}$ may result in the favoring by both $\mathrm{AgPtF}_{6}$ and $\mathrm{AgPdF}_{6}$ of the $\mathrm{M}(\mathrm{IV})$ and $\mathrm{Ag}(\mathrm{II})$ oxidation states. 


\subsection{References}

${ }^{1}$ B. Müller and R. Hoppe, Z. Anorg. Allg. Chem., 392 (1972), pp 37-41.

${ }^{2}$ W. E. Falconer, F. J. DiSalvo, A. J. Edwards, J. E. Griffiths, W. A. Sunder, and M. J. Vasile, J. Inorg. Chem., Supplement (1976), p 59.

${ }^{3}$ A. Tressaud, M. Winterberger, N. Bartlett, and P. Hagenmuller, C. R. Hebd. Seances Acad. Sci. Ser. C, 282 (1976), p 1069; A. Tressaud, F. Pintchovski, L. Lozano, A. Wold, and P. Hagenmuller, Mat. Res. Bull., 11 (1976), p 689.

${ }^{4}$ For Ti: E. O. Wollan, H. R. Child, W. C. Koehler, and M. K. Wilkinson, Phys. Rev., 112 (1958), p 1132; K. Knox, Acta Cryst., 13 (1960), p 507.

For V: P. Ehrlich and G. Pietska, Z. Anorg. Allg. Chem., 275 (1954), p 121; S. Siegel, Acta Cryst., 9 (1956), p 684.

For Cr: A. J. Jacobson, L. McBride, and B. E. F. Fender, J. Phys. C, 7 (1974), p 783.

For Fe, Co, Ru, Rh, Ir: M. A. Hepworth, K. H. Jack, R. D. Peacock, and G. J. Westland, Acta Cryst., 10 (1957), p 63.

For Ni: B. Žemva, K. Lutar, L. Chacón, M. Fele-Beuermann, J. Allman, C. Shen, and N. Bartlett, J. Am. Chem. Soc., submitted for review (1995).

For Mo: D. E. LaValle, R. M. Steele, M. K. Wilkinson, and H. L. Yakel, J. Am. Chem. Soc., 82 (1960), p 2433.

${ }^{5}$ N. Bartlett and J. W. Quail, J. Chem. Soc., (1961) p 3728. 
Chapter $8-A g^{I} M^{V} F_{6} v s . A g^{I I} M^{I V} F_{6}$

${ }^{6}$ National Bureau of Standards (U.S.), Circ. 539, 6 (1956), p 27.

${ }^{7}$ National Bureau of Standards (U.S.), Circ. 539, 6 (1956), p 42.

${ }^{8}$ N. Bartlett and J. Lohmann, J. Chem. Soc., (1964) p 619.

${ }^{9}$ National Bureau of Standards (U.S.) Monogr. 25 (1980), p 1757.

${ }^{10}$ R. D. W. Kemmitt, D. R. Russell, and D. W. A. Sharp, J. Chem. Soc., 844 (1963), pp $4408-4413$

${ }^{11}$ W. J. Casteel, Jr., Ph. D. Thesis, (1992) Chapter 7, U.C. Berkeley, and C. Shen, Ph. D. Thesis, (1992) Chapter 8, U.C. Berkeley.

${ }^{12}$ A. F. Kapustinskii, Quarterly Reviews, IX-X (1955), London: The Chemical Society, p 283. 


\section{Appendix}

\section{Estimating the Second Electron Affinity of $\mathrm{PtF}_{6}$}

\subsection{Discussion}

By comparing the energies involved in the formation of $\mathrm{Ag}^{\mathrm{I}} \mathrm{Pt}^{\mathrm{V}} \mathrm{F}_{6}$ with those involved in the formation of $\mathrm{Ag}^{\mathrm{II}} \mathrm{Pt}^{\mathrm{IV}} \mathrm{F}_{6}$, a hypothetical estimate for the second electron affinity of $\mathrm{PtF}_{6}$ may be derived. This calculation is only valid in the case that $\mathrm{AgPtF}_{6}$ is indeed a $\mathrm{Ag}^{\mathrm{II}}$ salt, as suggested by Müller and Hoppe ${ }^{1}$, which has yet to be confirmed. First, consider the following Hess' Law (or pseudo-Born Haber) cycle:

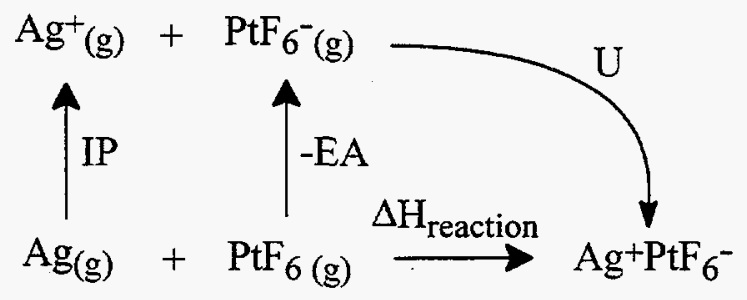

where IP is the first ionization potential of silver, EA is the first electron affinity of $\mathrm{PtF}_{6}$, and $\mathrm{U}$ is the lattice energy of $\mathrm{Ag}^{\mathrm{I}} \mathrm{Pt}^{\mathrm{V}} \mathrm{F}_{6}$. The heat of formation of $\mathrm{AgPtF}_{6}$ is given in Equation (3). 


$$
\Delta \mathrm{H}_{\text {reaction }}=\mathrm{IP}-\mathrm{EA}+\mathrm{U}
$$

Now consider the analogous Hess' Law cycle for the formation of the Ag(II) salt.

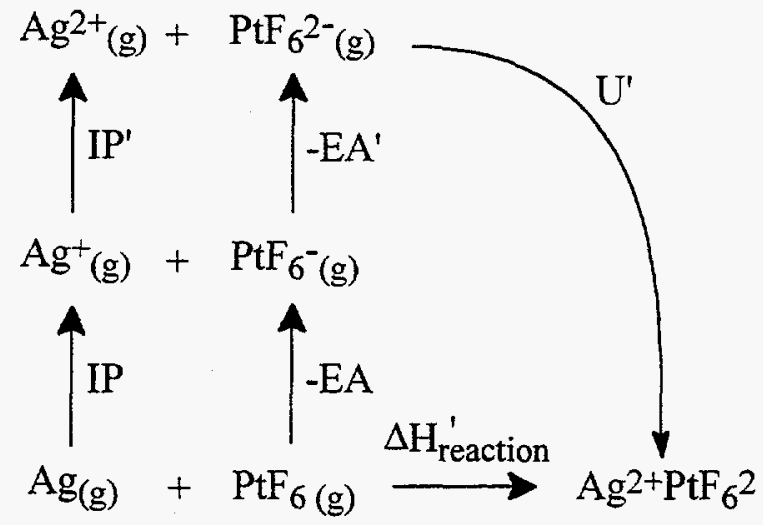

The heat of formation, in this case, is:

$$
\Delta \mathrm{H}_{\text {reaction }}^{\prime}=\mathrm{IP}+\mathrm{IP}^{\prime}-\mathrm{EA}-\mathrm{EA}^{\prime}+\mathrm{U}^{\prime}
$$

where IP' is the second ionization potential of silver, $\mathrm{EA}^{\prime}$ is the second electron affinity of $\mathrm{PtF}_{6}$, and $\mathrm{U}^{\prime}$ is the lattice energy for the formation of $\mathrm{Ag}^{\mathrm{II}} \mathrm{Pt}^{\mathrm{IV}} \mathrm{F}_{6}$. Since the entropy change is approximately the same for both reactions, $\Delta \mathrm{G}$ scales with $\Delta \mathrm{H}$. $\mathrm{Ag}^{2+} \mathrm{PtF}_{6}^{2-}$ is the preferred form, so the energy change for its formation must be more negative than for

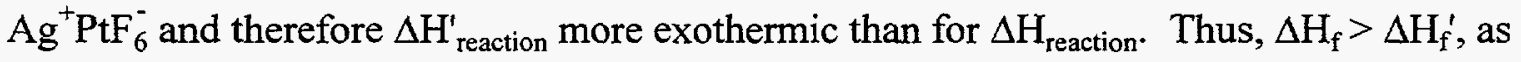
expressed in Equation (5).

$$
\begin{aligned}
I P-E A+U & >I P+I^{\prime}-E A-E A^{\prime}+U^{\prime} \\
U & >I P^{\prime}-E A^{\prime}+U^{\prime}
\end{aligned}
$$

Because the lattice energy, $U$, is proportional to the product of the charges $q_{+}$and $q_{-}$, we have

$$
U^{\prime} \approx 4 \times U
$$


which simplifies Equation (5) to

$$
\mathrm{EA}^{\prime}>\mathrm{IP}^{\prime}+3 \times \mathrm{U}
$$

The second ionization potential of silver is known ${ }^{1}$ to be $21.49 \mathrm{eV}$, or $496 \mathrm{kcal} \cdot \mathrm{mol}^{-1}$. And the lattice energy of an $\mathrm{Ag}^{\mathrm{I}} \mathrm{M}^{\mathrm{v}} \mathrm{F}_{6}$ compound having the formula unit volume of $\mathrm{AgIrF}_{6}$ may be estimated ${ }^{2}$ to be $-135 \mathrm{kcal} \cdot \mathrm{mol}^{-1}$. Therefore, the second electron affinity of $\mathrm{PtF}_{6}$, or $\mathrm{EA}\left(\mathrm{PtF}_{6}^{-}\right)$, must be greater than approximately $94 \mathrm{kcal} \cdot \mathrm{mol}^{-1}$. (By analogy, the same lower bound may be placed on the electron affinity of $\mathrm{PdF}_{6}^{-}$.) Although this value is in agreement with work performed by $\mathrm{Jha}^{3}$, in which the minimum electron affinity for $\mathrm{PtF}_{6}^{-}$was found to be $54 \mathrm{kcal} \cdot \mathrm{mol}^{-1}$, it represents an extraordinarily high electron affinity for an anion. Indeed, $94 \mathrm{kcal} \cdot \mathrm{mol}^{-1}$ exceeds even the electron affinity ${ }^{4}$ of atomic fluorine itself $\left(78.4 \mathrm{kcal} \cdot \mathrm{mol}^{-1}\right)$. 


\subsection{References}

${ }^{1}$ C. E. Moore, Natl. Ref. Data Ser., Natl. Bur. Stand., (1970) p 34.

${ }^{2}$ T. E. Mallouk, Ph.D. Thesis, (1983) Chapter 4, U.C. Berkeley.

${ }^{3}$ N. K. Jha, Ph.D. Thesis, (1965) Appendix IV, University of British Columbia.

${ }^{4}$ H. Hotop and W. C. Lineberger, J. Phys. Chem. Ref. Data, 4 (1975), p 539. 